\title{
MHD turbulence
}

\section{Andrey Beresnyak ${ }^{1}$}

Received: 8 August 2018 / Accepted: 16 August 2019 / Published online: 10 September 2019 (c) The Author(s) 2019

\begin{abstract}
We review the current status of research in MHD turbulence theory and numerical experiments and their applications to astrophysics and solar science. We introduce general tools for studying turbulence, basic turbulence models, MHD equations and their wave modes. Subsequently, we cover the theories and numerics of Alfvénic turbulence, imbalanced turbulence, small-scale dynamos and models and numerics for supersonic MHD turbulence.
\end{abstract}

Keywords Astrophysics · Magnetohydrodynamics · MHD turbulence $\cdot$ Numerical simulations · Numerical methods · Turbulence

\section{Contents}

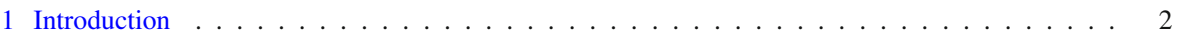

2 MHD turbulence in astrophysics . . . . . . . . . . . . . . . . . . . . . . 4

3 Statistical description of turbulence . . . . . . . . . . . . . . . . . . . . . . 10

4 Kolmogorov cascade model . . . . . . . . . . . . . . . . . . . . . . . . . . . 14

4.1 Lagrangian spectrum . . . . . . . . . . . . . . . . . . . . . . 16

4.2 More general Kolmogorov phenomenology . . . . . . . . . . . . . . . . . . . . . 17

4.3 Scaling convergence in turbulence: numerics and experiments . . . . . . . . . . . . . . . 17

5 MHD equations, modes . . . . . . . . . . . . . . . . . . . . . . . . . 19

6 Numerical methods to simulate MHD turbulence . . . . . . . . . . . . . . . . . . . . 20

6.1 Pseudospectral codes . . . . . . . . . . . . . . . . . . . . . . . 20

6.2 Finite difference codes . . . . . . . . . . . . . . . . . . . . . . . . . . . . . . . . . . . . . .

6.3 Finite volume codes with Riemann solvers . . . . . . . . . . . . . . . . . . . . . . 21

6.4 Lagrangian codes . . . . . . . . . . . . . . . . . . . . . . . . . . . . . . . . . . . . . . . . . . . . . . . . . . . . .

7 Theory of Alfvénic turbulence . . . . . . . . . . . . . . . . . . . . . . 21

7.1 From weak to strong turbulence . . . . . . . . . . . . . . . . . . . . . . 22

7.2 Reduced MHD approximation . . . . . . . . . . . . . . . . . . . . . . . . . . . . . . . . . . . . . . . . . . . .

7.3 Strong MHD turbulence . . . . . . . . . . . . . . . . . . . . . . . . . . . 25

7.4 Numerics: perpendicular spectrum . . . . . . . . . . . . . . . . . . . . . . 27

7.5 Numerics: parallel spectrum _. . . . . . . . . . . . . . . . . . . . . 30

7.6 Numerics: anisotropy . . . . . . . . . . . . . . . . . . . . . . . . 32

Andrey Beresnyak andrey.at.astro@gmail.com

1 Plasma Physics Division, U.S. Naval Research Laboratory, Washington, DC, USA 
7.7 Dynamic alignment models . . . . . . . . . . . . . . . . . . . . . . 33

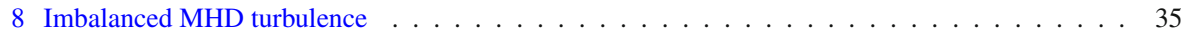

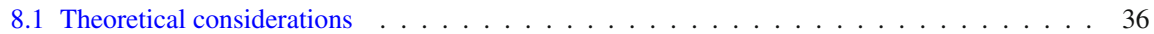

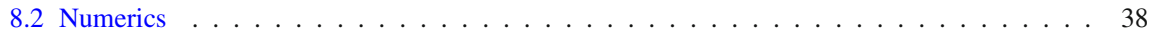

9 MHD dynamo . . . . . . . . . . . . . . . . . . . . . . . . . . . . . 40

9.1 Kinematic dynamo . . . . . . . . . . . . . . . . . . . . . . . 41

9.2 Nonlinear dynamo . . . . . . . . . . . . . . . . . . . . . . . . . . . . . . . . . . . . . . . . . . . . . . .

9.3 Numerical results . . . . . . . . . . . . . . . . . . . . . . . . . . . . . . . . 4 44

9.4 Caveats in simulating astrophysical dynamo . . . . . . . . . . . . . . . . . . 45

9.5 Application to galaxy clusters . . . . . . . . . . . . . . . . . . . . . . . . . . . . . . . . . . . . . . . 46

10 Compressible and supersonic turbulence . . . . . . . . . . . . . . . . . . . . 47

10.1 Decomposition into modes . . . . . . . . . . . . . . . . . . . . . . . . . . . . . . . . . . . . .

10.2Decomposition in real space . . . . . . . . . . . . . . . . . . . . . . 49

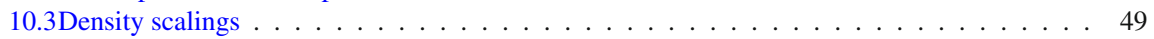

11 Turbulence driven by magnetic field . . . . . . . . . . . . . . . . . . . 51

12 Conclusion .............................. 53

References . . . . . . . . . . . . . . . . . . . . . . . 54

\section{Introduction}

Turbulence is a time-dependent, stochastic flow commonly found in fluids with low viscosity. Shear viscosity is associated with microscopic phenomena, and the size of the turbulent system is much larger than the viscous scale. Turbulence develops from laminar flow due to instabilities and has many degrees of freedom. Despite its complexity, researchers investigate turbulence because of practical importance. The effect of turbulence is not only unpredictability of each realization of the flow, but often very important, quantifiable and predictable effects which are attractive to scientists and engineers. For example, ideal equations of motion, such as Euler's equation can be used, under certain conditions, to derive conservation laws. The conservation of energy and the conservation of the velocity circulation along the path frozen into the fluid (Kelvin's theorem) are notable examples of these "ideal invariants". However, physicists realized very early on that moving through the fluid involves drag and the loss of energy. Despite there is always a stationary ideal flow that produces zero drag (d'Alembert paradox), in practice such flows are not realizable due to instabilities and finite viscosity. Turbulence research elucidated this energy loss process and argued that it could happen for arbitrarily small viscosity due to the conserved quantity forming a "cascade" through scales finally dissipating on sufficiently small scales (RichardsonKolmogorov picture). Likewise, Kelvin's circulation theorem is broken for flow around the wing, making possible lift force and the airplane flight.

Compared to turbulence on Earth, astrophysical turbulence is characterized by even larger scale separation between the problem size and the dissipative size, this makes turbulence in space almost unavoidable. Unlike the flows of non-conductive fluids on Earth, well-described by the Navier-Stokes equations, astrophysics deals with flows of ionized plasmas which, in most cases, can be considered perfectly conducting. These flows are described by equations with currents, magnetic fields and the Lorentz force, namely magnetohydrodynamic (MHD) equations.

In most astrophysical environments magnetic fields are observed and often are dynamically important. In our Galaxy, similar to other spiral galaxies, the magnetic 
field has a regular as well as random components. The value of the magnetic field, around $5 \mu \mathrm{G}$, suggests equipartition between magnetic and kinetic energies. In the galaxy clusters, the magnetic field is of order $1-3 \mu \mathrm{G}$, which is around $1 / 20$ th of the equipartition. Another example is the convective cell on the Sun. Its magnetic field is also somewhat close to the equipartition with the motions. Our Universe would have been very boring if it had both electric and magnetic charges, so that both electric and magnetic fields are screened out on large scales. Fortunately, this is not the case, the magnetic field and large-scale motions result in the acceleration of particles and the Universe if filled with non-thermal radiation in all wavebands.

Considering the space is filled with ionizing radiation it is not surprising that astrophysical plasmas are well-conductive. However, do they always have to be wellmagnetized as well? The process of generation or amplification of the field is known as a dynamo, and this process seems to work sufficiently fast to do its job everywhere. If we start with zero magnetic field in the MHD equations, this produces precisely zero field in the future in an apparent contradiction with the ubiquity of magnetic fields. Do we always have to rely on primordial magnetic fields or the effects beyond simple MHD equations? In this review, among other things, we will emphasize that the growth of magnetic energy can be described in a framework somewhat similar to the loss of kinetic energy in the nearly ideal hydrodynamic flows. In other words, fast dynamo is an inherent property of turbulence.

Magnetic turbulence is also the primary cause of accretion onto gravitating objects, in particular accretion onto black holes is estimated to be the most potent source of energy in the Universe, exceeding thermonuclear burning in stars. Thin stationary accretion disks in a Keplerian potential are hydrodynamically stable, so in order to generate accretion one has to rely on the excitation of the the magnetic degree of freedom, the problem known as magnetorotational instability (MRI). Related to MRIunstable disks are astrophysical jets, highly collimated flows perpendicular to the accretion disks in which magnetic field is essential in the process of launching and collimation of the flow.

The Alfvén theorem of perfectly conducting magnetohydrodynamics states that magnetic field lines are perfectly frozen into the conductive fluid, which places a severe restriction on the process of the so-called magnetic reconnection - the change of topology of the magnetic configuration by magnetic field lines crossing and moving through magnetic null. By way of restricting such change, the Alfvén theorem also precludes fast release of magnetic energy in highly conductive environments. This is in gross contradiction with high-energy phenomena above the solar surface known as Xray flares. Again, turbulence comes to the rescue and allows for the radical breaking of the Alfvén theorem, even in near-perfectly conducting fluids, very much like breaking of Kelvin's theorem of hydrodynamics.

In recent years the two traditional pillars of physics - the theory and the experiment has been complemented by a new method, numerical simulations. Numerics is valuable because it covers the gap between the real world, experimental data, and the idealized theory, a gap often being too great and precluding discovery. Numerics solves idealized equations directly, in this aspect it is similar to theory. On the other hand, numerics may be referred to as "numerical experiment", measurement of physical quantities without invoking much of the assumptions or prerequisites. Compared to the real- 
life experiment, in numerics, it is easier to study idealized cases such as statistically homogeneous or statistically stationary turbulence for which the theory has something to say At the same time numerics reduces almost infinite space of theoretical ideas by weeding out theories which are incompatible with numerical measurements. In the studies of turbulence, the strength of numerics is manifested in the high statistical accuracy of the results, especially on small scales. Compared to the experimental measurements or observations which have high statistical and systematic uncertainties this helps to discriminate between theories and make quicker progress.

One type of numerics, direct numerical simulations (DNS) will be highlighted in this review. DNS refers to "fully resolved" numerical experiment, where numerics is very accurate and faithfully reproduce solutions of the original equations on all scales. On the other end, there are Implicit Large Eddy Simulations (ILES), calculations aiming to get the large-scale features of the flow correct without caring about the details of the dissipation in small-scale turbulence or shocks. These are very common is astrophysics, allowing to simulate large objects which are indeed out of reach of DNS, however as we will show in the dynamo section this should be used with caution.

This, primarily theoretical, review mostly deals with homogeneous (although usually anisotropic) turbulence. Homogeneous models benefit from the opportunity to average quantities over volume, instead of averaging over ensemble (see more in Sect. 3). Few examples of important physical problems involving inhomogeneous turbulence: (1) large-scale dynamo, where inhomogeneity is required to break the statistical symmetry of turbulence, typically mirror symmetry, to produce large-scale magnetic fields, see also Sect. 9; (2) generation of imbalanced turbulence with a localized source of perturbations, see also Sect. 8; (3) large-scale dynamics of expanding solar wind; (4) magnetic shear as a driver of turbulence, see Sect. 11; (5) MRI, mentioned above. Very often inhomogeneous problems are treated with the scaleseparation technique, where turbulence is described is some sort of "local box" approximation, within the box it is assumed homogeneous, but have overall driving, for example, shear boundary condition, as in the case of MRI.

\section{MHD turbulence in astrophysics}

Turbulence results from instabilities of large-scale fluid motions experiencing low friction forces. Dimensionless Reynolds number characterizes the relative importance of viscosity

$$
\operatorname{Re}=L V / \nu,
$$

where $L$ is the characteristic scale of the flow, often called "outer scale," e.g., the diameter of a jet, $V$ is its velocity, and $v$ is fluid kinematic viscosity (in units of $[L]^{2} /[T]$ ). Likewise, one can introduce similar magnetic Reynolds number

$$
\operatorname{Re}_{m}=L V / \eta
$$


where $\eta=c^{2} / 4 \pi \sigma$ is magnetic diffusivity, $c$ is a speed of light, and $\sigma$ is a conductivity and Lundquist number

$$
S=L v_{A} / \eta
$$

where

$$
v_{A}=B / \sqrt{4 \pi \rho}
$$

is Alfvén speed, in units of velocity. $\mathrm{Re}, \mathrm{Re}_{m}$ and $\mathrm{S}$ are typically very large in astrophysics, meaning that viscous and resistive effects should be very small, the numbers of order $10^{10}$ or larger are common. A notable caveat of this simple picture is that astrophysical plasmas are very often collisionless and the rigorous derivation of simple diffusive transport coefficients, such as Chapman-Enskog expansion, simply fails. So, our transport coefficients refer to some "effective" diffusivities, the physical meaning of which can be understood as follows. In molecular physics, the kinematic viscosity can be estimated as $\bar{u} l$, a product of thermal speed and the mean free path. It is then clear that the Reynolds number is the ratio of the product of velocity and scale corresponding to macro- and micro-scales. A suitably chosen "effective" mean free path will allow estimating $\mathrm{Re}$ and the scale at which fluid motion transitions into the dissipative or dispersive regime, for example, the Kolmogorov scale that we introduce in Sect. 4. Such trickery works extremely well for fluid flows with turbulence or shocks and also the logic behind ILES. Throughout this review, the reader will see many examples of the so-called scale locality of turbulence in action, in particular, large-scale properties of the flow are insensitive to the diffusivities.

One way to understand astrophysical turbulence is to understand the source of energy and how it is converted to turbulent motions. The biggest source of energy in the Universe is gravity. Turbulence can be driven by cosmological flows when gravity amplifies initially small density perturbations and cause structure formation. This is a very slow process, however, and typically dynamical times of voids are less then unity, in units of the age of the Universe, the dynamical times of filaments (superclusters) are of order unity, while dynamical times in the intra-cluster medium (ICM) of the galaxy clusters are of order 20 , meaning they are expected to be turbulent. The size of a typical large galaxy cluster is of the order of several megaparsecs (Mpc), and the main source of turbulence is the infalls of large chunks of matter into it, so-called major mergers. While the direct evidence of kinetic turbulence motions in clusters is still scarce, the evidence of magnetic fields produced by dynamo action is available, see, e.g., Fig. 1.

The source of turbulence in the plasma of ordinary galaxies, called interstellar medium (ISM), is likely multiple. At the present time, collisions with other galaxies are fairly rare. Several other mechanisms of driving turbulence can be identified, however: (a) galactic disk is conductive and as such is subject to MRI, (b) supernova explosions expand to the scale of several parsecs colliding with density inhomogeneities of the ISM, producing large-scale irregular motions, (c) ISM turbulence is subject to Parker's instability when volumes of the ISM which are more magnetized and filled with more cosmic rays (CRs) are more buoyant compared to volumes poorly magnetized and scarce in CRs, so convective instability against the gravity of the disk ensues, (d) CRs and starlight heat ISM at the same time cools itself by atomic and molecular emission on lower frequencies, and this produces thermal instability in the gas, (e) jets 


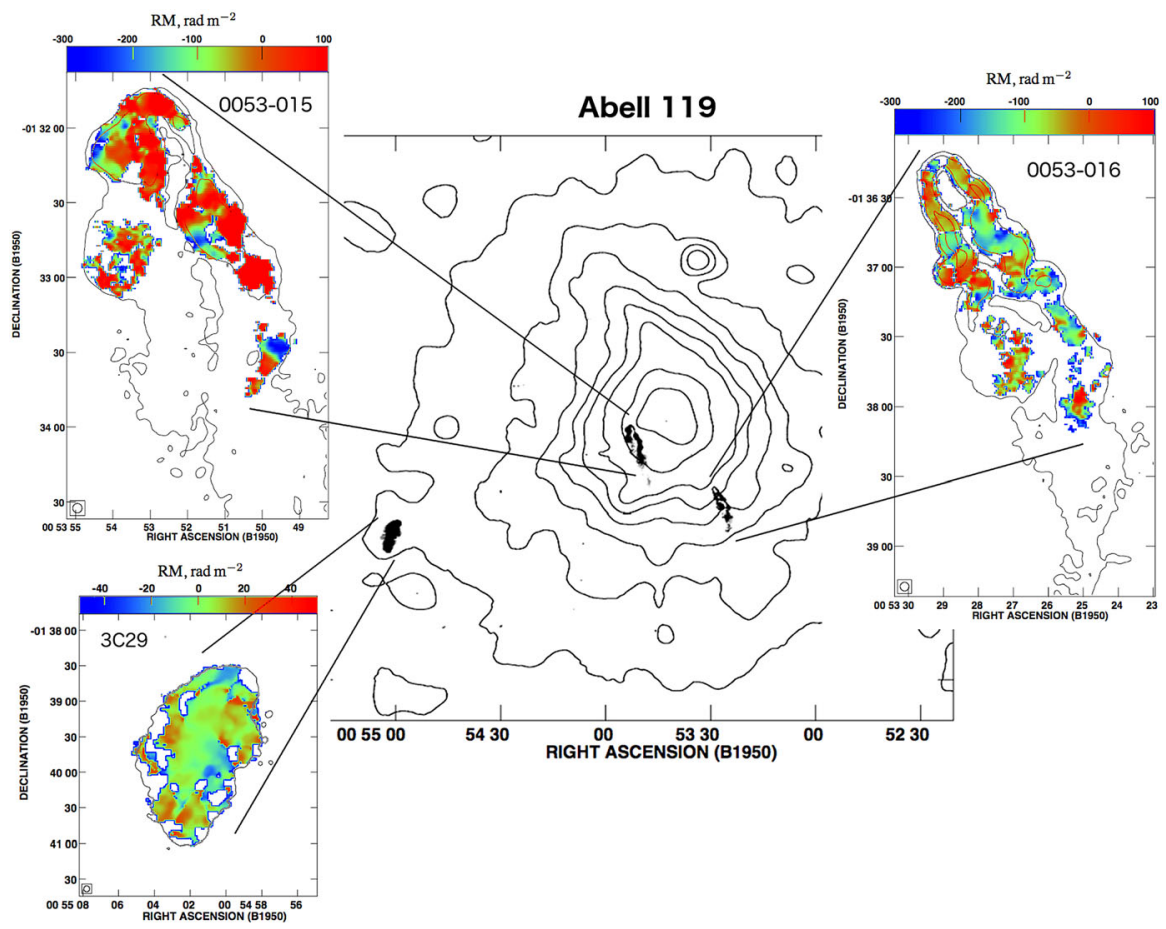

Fig. 1 Faraday rotation measure maps of radio sources within a galaxy cluster. Adapted from Feretti et al. (1999), Dolag et al. (2009). The cluster electrons act as a foreground for the radio source. These maps indicate random magnetic fields of several $\mu \mathrm{G}$ in the cluster, changing on scales of $10-40 \mathrm{kpc}$

and winds from young stars collide with ISM inhomogeneities producing irregular motions, (f) at high redshifts also accretion and merger. The complexity of the ISM turbulence is rather overwhelming, and we refer to Mac Low and Klessen (2004), McKee and Ostriker (2007) for further reading. The evidence of turbulence present in the ISM was compiled from different sources by Armstrong et al. (1995) and sometimes is referred to as a "Big power law in the sky", see Fig. 2. One amusing property of ISM turbulence is the large-scale dynamo, which produces the magnetic field on the scales of the disk, tens of kpc, while the outer scale of turbulence is only $10-100 \mathrm{pc}$. The evidence of large-scale magnetic fields in other galaxies is abundant, see e.g., Fig. 5, while the evidence of fluctuating component of the magnetic field is mostly limited to our own Galaxy, due to the limited resolution of the observations.

Another source of kinetic energy derived from gravity is jets from disks around black holes. This process is actually more efficient than thermonuclear burning in stars, including explosive burning in supernovae. The disk is conductive and unstable to MRI, turbulence in the disk creates rather large-scale structures, see, e.g., Fig. 3 and magnetic fields. The jet may be driven from the rotating black hole directly or may be driven centrifugally at larger distances by gas escaping along open magnetic field lines. 
Fig. 2 Fluctuation of density in the interstellar medium (ISM), "big power law in the sky" (Armstrong et al. 1995; Chepurnov and Lazarian 2010). This 3D spectral density with slope of $-11 / 3$ correspond to Kolmogorov's 1D spectrum with slope of $-5 / 3$. Whether this power law, obtained from a variety of observations of different ISM components, is part of a single turbulent cascade is still an open question. Image reproduced with permission from Chepurnov and Lazarian (2010); copyright by AAS

Fig. 3 Density, shown in color, in a simulated accretion disk around a black hole, subject to MRI. The length unit is two gravitational radii of the black hole. Image reproduced with permission from Jiang et al. (2019); copyright by AAS
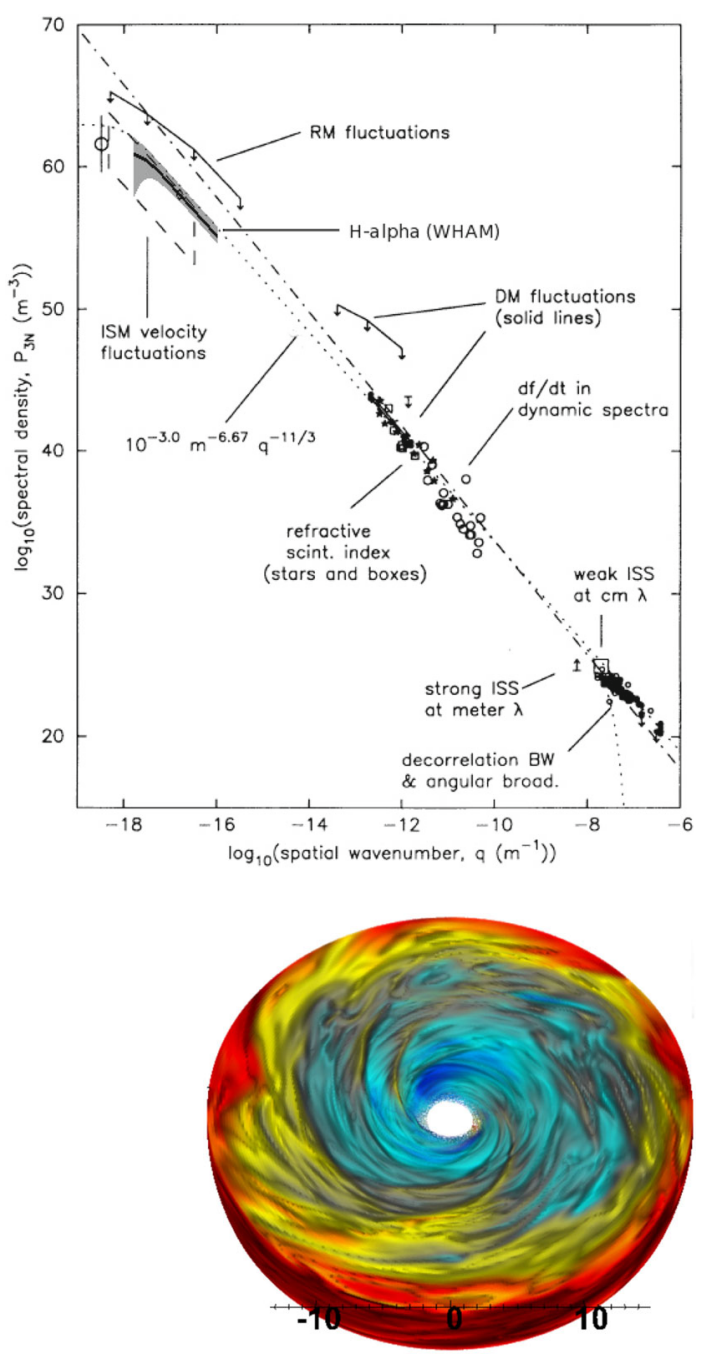

In the solar system, we can make in-situ measurements in the solar wind, the flow of tenuous magnetized plasma emitted from the Sun at speeds $400-800 \mathrm{~km} / \mathrm{s}$ and propagating outwards to the boundaries of the solar system. Such direct measurements of the solar wind parameters and fluctuations in different regions from 0.3 to $5 \mathrm{AU}$ distance to the Sun are especially valuable because they convey much more precise information about turbulent fluctuations compared to astrophysical observations of ISM and ICM mired by limited resolution and projection effects. Ion and electron counters on the satellite provide information about the flow, while magnetometers measure magnetic fields. Solar wind properties widely vary depending on the flow angle with respect to the ecliptic, see Fig. 4. The measurements by a single spacecraft represent time-sequence, demonstrating fluctuations on timescales from days to seconds, which can be Fourier-analyzed. The velocity of the solar wind is much larger than the local 
Fig. 4 Density and speed of the solar wind recorded by Ulysses/SWOOPS. Image reproduced with permission from McComas et al. (2000); copyright by AGU

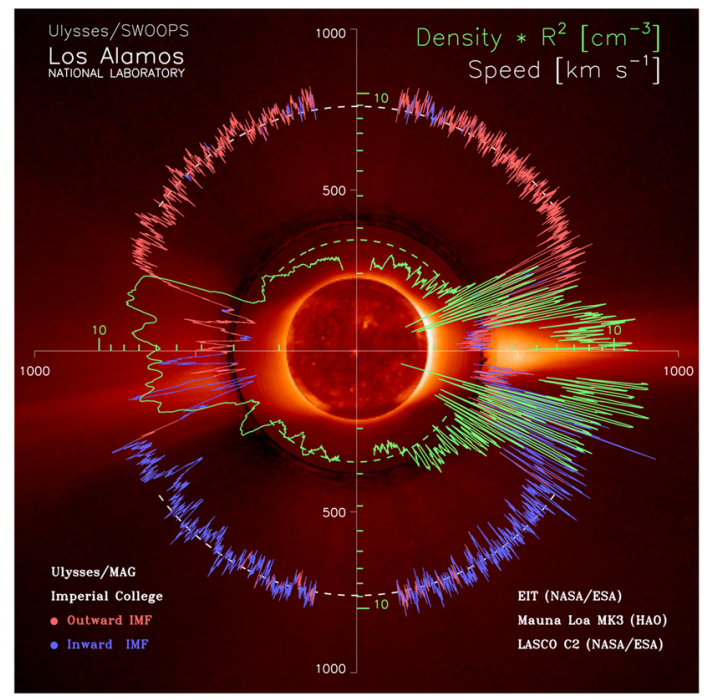

Fig. 5 B-vectors in spiral galaxy IC 342 observed at $6 \mathrm{~cm}$ (VLA and Effelsberg) overlaid on the Kitt Peak Observatory image (credit: T. A. Rector, University of Alaska Anchorage, and H. Schweiker, WIYN and NOAO/AURA/NSF). Image reproduced with permission from Beck (2015); copyright by ESO

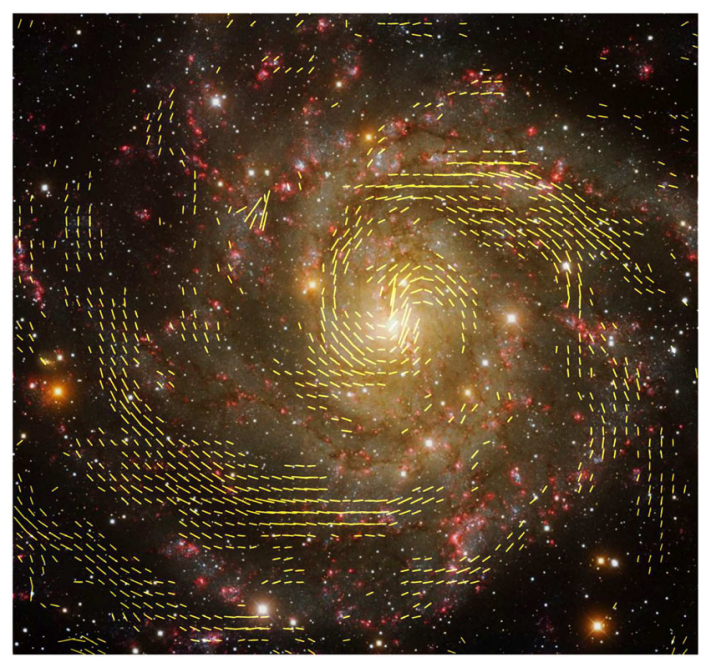

Alfvén speed of around $30 \mathrm{~km} / \mathrm{s}$ so that the measurement can be interpreted as the spacial spectrum, see, e.g., Fig. 6 for spectra obtained from measurement by Helios 2 spacecraft. The $f^{-1}$ part of the spectrum corresponds to the shot-noise statistics of features emitted by the Sun, while the $f^{-5 / 3}$ part is the evidence of well dynamically evolved turbulence, the characteristic timescales on these scales are indeed shorter than the time of flight from the Sun.

The Sun's outer envelope transports energy to the surface by convection, also generating magnetic fields in the process. The magnetic field is distributed extremely unevenly on the surface reaching several kilogauss in sunspots. Sunspots are connected by magnetic arcs visualized by structure because hot plasma has high thermal conductivity along the field and low conductivity perpendicular to it. Interaction of 


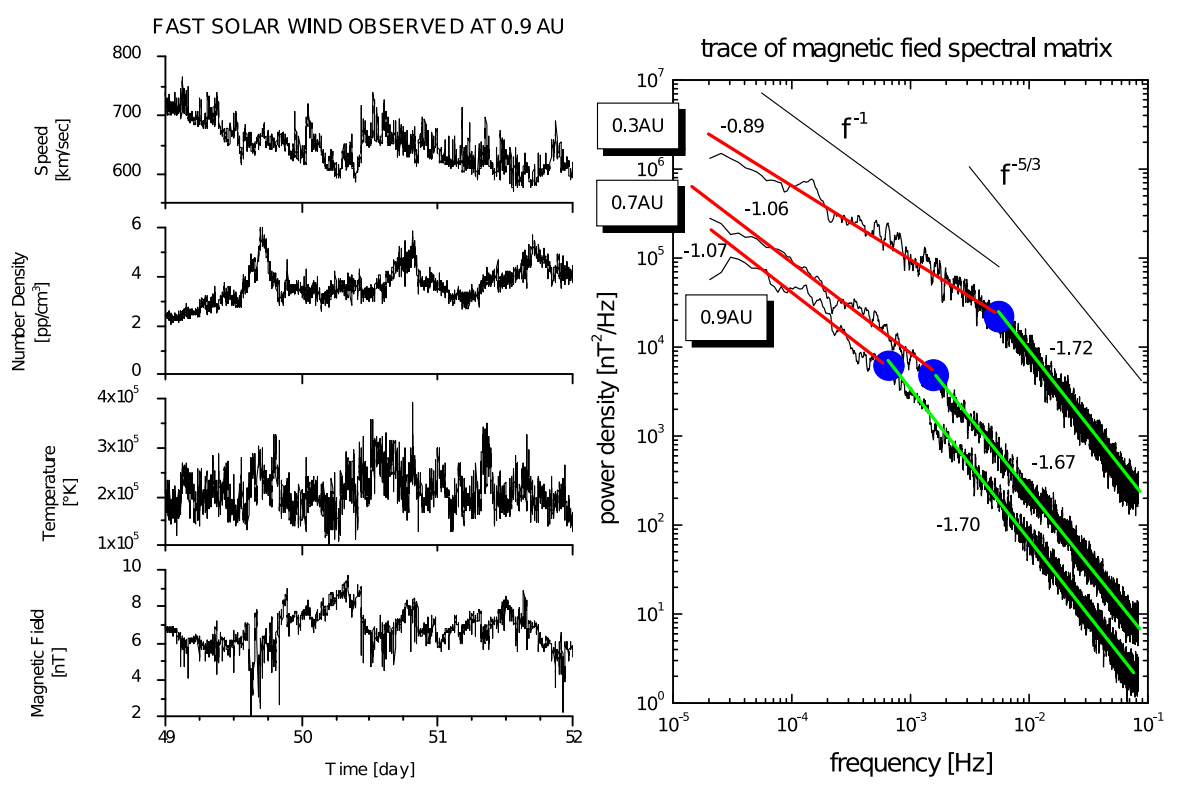

Fig. 6 Left: A sample of fast solar wind at a distance of 0.9 AU measured by the Helios 2 spacecraft. Right: Power density spectra of magnetic field fluctuations observed by Helios 2 between 0.3 and 1 AU. Image reproduced with permission from Bruno and Carbone (2013); copyright by the authors

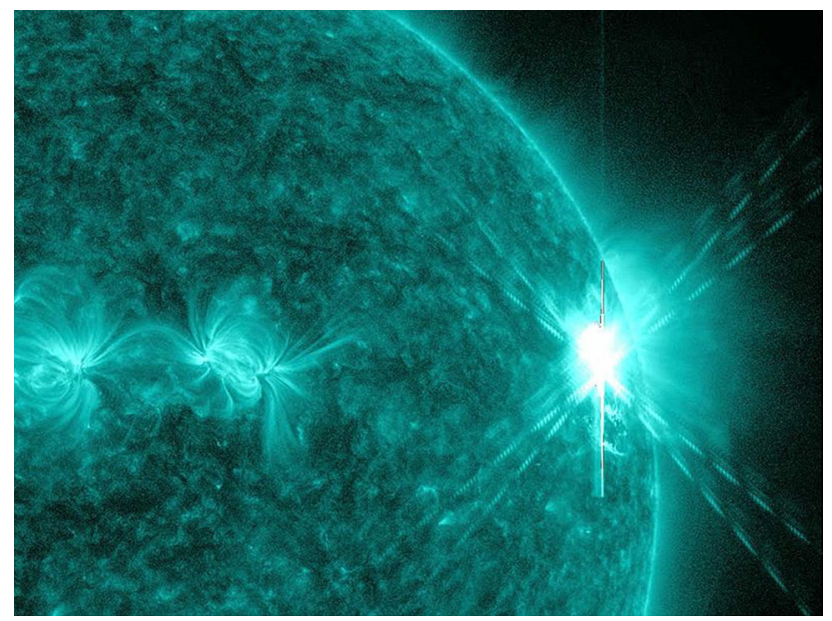

Fig. 7 X-class solar flare caused by reconnection of large arcs of magnetic field above the solar surface (NASA/SDO)

strong magnetic flux tubes above the solar surface leads to magnetic reconnection which results in two spectacular phenomena: X-ray flares (see Fig. 7) and coronal mass ejections (CME). It is conjectured that reconnection and the release of magnetic energy are due to the thin current sheet at the intersection of flux tubes becomes unstable and generate turbulence, see Sect. 11. 
Fig. 8 Simulated MHD

turbulence visualized by magnetic field magnitude shown in grayscale. This statistically homogeneous, isotropic turbulence with zero net magnetic flux was driven by volumetric force to statistically stationary state, one snapshot of which is shown on the picture. Image reproduced with permission from Beresnyak and Lazarian (2019); copyright by De Gruyter

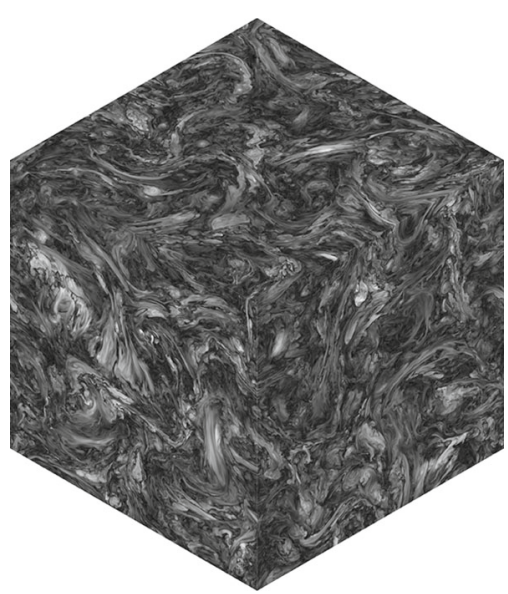

\section{Statistical description of turbulence}

In this section, we will briefly introduce a statistical description. For a more in-depth review of this subject, we highly recommend the monograph by Monin and Yaglom (1975). While single realizations of turbulent flow are chaotic and unpredictable, there's some order. This order can mostly be described statistically, at the same time engineers and scientists are not interested in individual realizations, but rather in averaged quantities, such as an averaged lift of an airfoil. Turbulence is a volumefilling and persistent process, its realizations filling configuration space densely so that the statistical ensemble measurements sometimes can be replaced with timeand volume-averaging (ergodic hypothesis.) The theory relies typically on ensemble averaging, but numerical experiments mostly use volume and time averaging, see Fig. 8. We will designate averaging as \langle\rangle without specifying whether its statistical, time or volume averaging.

The spatial variability of a physical variable, e.g., $\mathbf{v}(\mathbf{r})$, over some scale $l$ can be described as some function of the difference of $\mathbf{v}$ between points separated by a distance $l$. Second-order statistics can be related to energy, for example, the secondorder structure function (SF) of velocity,

$$
\mathrm{SF}^{2}(\mathbf{l})=\left\langle(v(\mathbf{r}-\mathbf{l})-v(\mathbf{r}))^{2}\right\rangle .
$$

In the limit of large $l$ this equals to four times kinetic energy, while for smaller $l$ it is four times "characteristic energy" on this scale and all smaller scales. Fourier-transformed $\mathrm{SF}$ can be related to "energy spectrum" $E(k)$ (see below). The energy spectrum is the energy distributed in wavenumber space, with $d E=E(k) d k$ being the energy at a particular wavenumber and $\int E(k) d k$ being total energy. When turbulence is statistically self-similar we expect a power-law scaling of statistical quantities, e.g., $E(k)$.

The SF above represents the sum of the longitudinal and transverse components of the velocity with respect to direction perpendicular and parallel to $\mathbf{l}$. Naturally, longitudinal and transverse functions can be calculated separately. The longitudinal 
SF is historically important in experimental research of hydrodynamic turbulence due to being the primary quantity measured by the heated wire technique. The large-scale flow $\mathbf{v}_{0}$ around the wire carries smaller fluctuations which cause fluctuations of the absolute value of $\mathbf{v}$, which is what is measured by the changing resistance of the wire, however, fluctuations in $|v|$ are mostly due to fluctuations parallel to the average $\mathbf{v}_{0}$. The Taylor hypothesis assumes that time variations correspond to variations in space, i.e., measurements separated by $t$ correspond to $\mathbf{l}=\mathbf{v}_{0} \delta t$. Thus we measure only the component parallel to $\mathbf{l}$. In the solar wind measurements, all three vector components are recovered so that the transverse, longitudinal and full structure functions can be calculated.

In the case of isotropic turbulence $\operatorname{SF}(\mathbf{l})$ is only a function of $l$, MHD turbulence is not isotropic, however, so there is a wider variety of structure functions that we can measure. However, as we show below, in the reduced MHD limit there is a particular structure function which plays the similar role as the isotropic SF in hydrodynamics, the perpendicular SF

$$
\mathrm{SF}_{\perp}^{2}(l)=\left\langle\left(w^{ \pm}(\mathbf{r}-l \mathbf{n})-w^{ \pm}(\mathbf{r})\right)^{2}\right\rangle_{\mathbf{r}}
$$

where $\mathbf{n}$ is a vector perpendicular to the magnetic field.

The turbulent quantity $u(\mathbf{r})$ can be Fourier-transformed:

$$
u(\mathbf{r})=\int e^{i \mathbf{k} x} d \hat{u}(\mathbf{k}),
$$

with the square of the transform called power spectrum:

$$
F(\mathbf{k}) d \mathbf{k}=\left\langle|d \hat{u}(\mathbf{k})|^{2}\right\rangle
$$

This function can be integrated over the sphere in k-space, e.g., if $F(\mathbf{k})$ depends only on the magnitude of $\mathrm{k}$ we have $E(k)=4 \pi k^{2} F(k)$, the resulting quantity we will call three-dimensional spectrum. Similar procedure is possible when sampling the field along the line, i.e., in one dimension, this quantity will be called a one-dimensional spectrum $E_{1}(k)=2 F_{1}(k)$. Note that 2 comes from $F(k)$ being defined for positive and negative wavenumbers. $E(k), E_{1}(k)$ can be related in isotropic case by

$$
E_{1}(k)=\int_{k}^{\infty} E\left(k_{1}\right) \frac{d k_{1}}{k_{1}},
$$

while the above mentioned parallel one-dimensional spectrum, which we designate as $E_{\|}(k)$ where only parallel component of velocity is used, for a solenoidal isotropic field:

$$
E_{\|}(k)=\int_{k}^{\infty} E\left(k_{1}\right)\left(1-\frac{k^{2}}{k_{1}^{2}}\right) \frac{d k_{1}}{k_{1}},
$$

so that if $E(k)$ is a power law $E(k) \sim k^{\gamma}$ then $E(k)=-\gamma E_{1}(k)$ and $E(k)=$ $\gamma(\gamma-2) E_{\|}(k)$. In practice, spectra are never exact power laws, so the shape of these 
Fig. 9 Three types of spectra from MHD numerical simulation. $E(k)$-solid, $E_{1}(k)$-dashed, $E_{\|}(k)$-dash-dotted. The upper and lower simulations differed in the shape of the elementary cell and the influence of the numerical error. Note the different shapes of the three types of spectra, despite all three spectra convey essentially the same information about energy content of turbulence on different scales. Image reproduced with permission from Beresnyak and Lazarian (2015); copyright by Springer

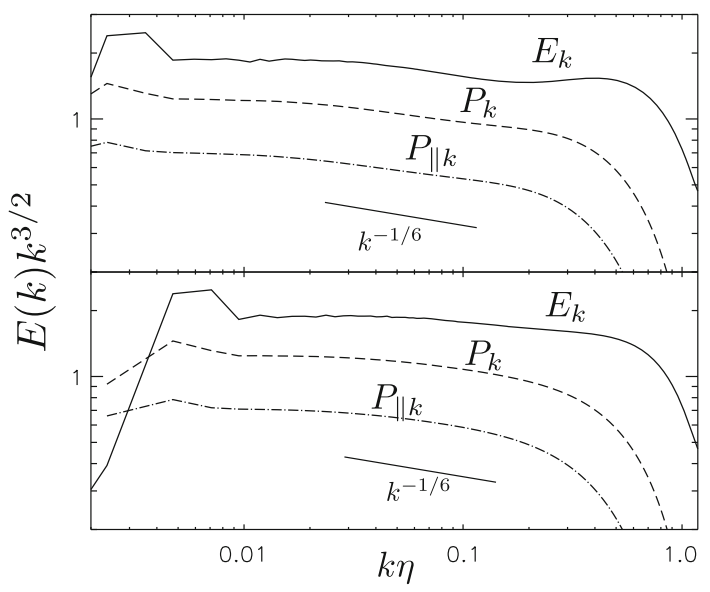

spectra are different. Figure 9 shows three types of spectra from a simulation of MHD turbulence.

Spectra and structure functions have one-to-one correspondence by Fourier transforms:

$$
\begin{aligned}
& \operatorname{SF}^{2}(r)=2 \int_{0}^{\infty}\left(1-\frac{\sin k r}{k r}\right) E(k) d k, \\
& \operatorname{SF}^{2}(r)=2 \int_{0}^{\infty}(1-\cos k r) E_{1}(k) d k .
\end{aligned}
$$

If the spectrum has a power-law dependence $k^{\alpha}$, then by substitution $k=x / r$ we obtain

$$
\operatorname{SF}^{2}(r) \sim r^{-1-\alpha},
$$

provided that remaining dimensionless integral converge. This relation is satisfied for $\alpha$ between -3 and -1 .

From a statistical viewpoint, turbulence self-similarity, i.e., the assumption that turbulence has a single-fractal structure, would mean that for structure functions of arbitrary orders $n$ and $m$ one can write:

$$
\left(\mathrm{SF}^{n}(r)\right)^{1 / n} \sim\left(\mathrm{SF}^{m}(r)\right)^{1 / m}
$$

Some exact relations for structure functions in turbulence are known for hydrodynamics and MHD, which helps to test numerics. In the subsequent section, we explain in more detail the concept of the inertial range-a range of scales where energy is being overall conserved and is being transferred from one scale to another. From the dynamical viewpoint, these are scales at which dissipation term can be ignored, and the energy is only injected from large-scale motions but not from an external force. 

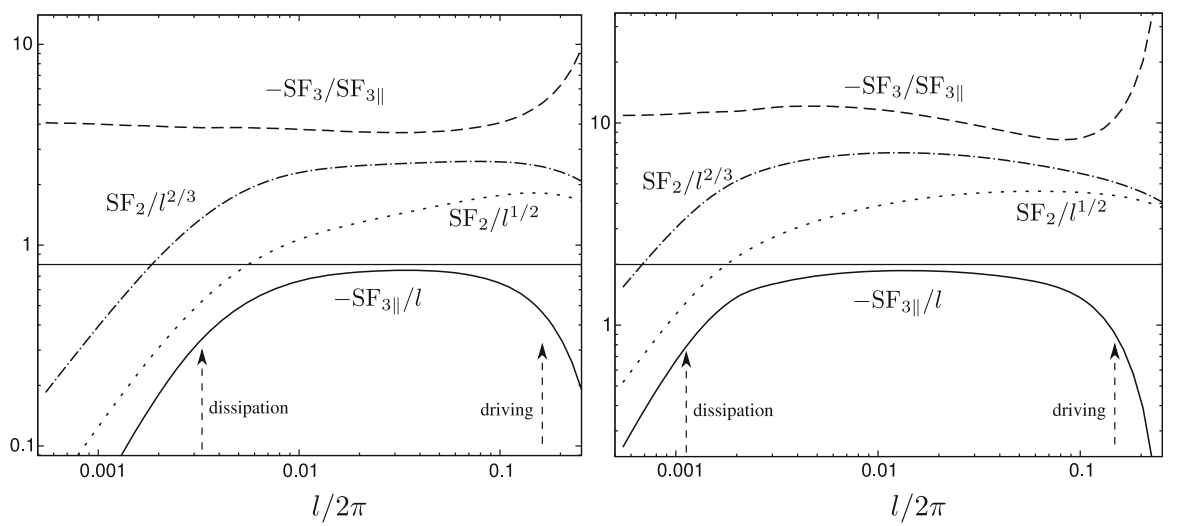

Fig. 10 Different structure functions vs the distance $l$, measured in hydrodynamic (left) and MHD (right) simulations. Solid lines show $-\mathrm{SF}_{3 \|} / l \varepsilon$. The influence of driving and dissipation is minimized in the point where $-\mathrm{SF}_{3 \|} / l \varepsilon$ is closer to its theoretical value. The dashed line indicates the ratio of the third order signed and unsigned SFs as a test for self-similarity. Dotted and dash-dotted lines indicate second-order structure functions, compensated by $l^{1 / 2}$ and $l^{2 / 3}$ correspondingly, in arbitrary units. Here, $l^{2 / 3}$ is the RichardsonKolmogorov scaling and $l^{1 / 2}$ is the scaling that appears in Kraichnan DIA model for hydrodynamics or Iroshnikov-Kraichnan model for MHD. Image reproduced with permission from Beresnyak and Lazarian (2015); copyright by Springer

The Kolmogorov -4/5 law relates a parallel signed structure function for velocity in the inertial range with the turbulent dissipation rate:

$$
\mathrm{SF}_{\| h}^{3}(l)=\left\langle\left(\delta v_{l \|}\right)^{3}\right\rangle=-\frac{4}{5} \varepsilon l
$$

Another exact relation, similar to the Yaglom's -4/3 law for incompressible hydro exists for axially symmetric MHD turbulence:

$$
\operatorname{SF}_{\|}^{3}(l)=\left\langle\delta w_{l \|}^{\mp}\left(\delta w_{l}^{ \pm}\right)^{2}\right\rangle=-2 \varepsilon l,
$$

where $l$ is taken perpendicular to the axis of statistical symmetry-the direction of the mean magnetic field B (Biskamp 2003; Politano and Pouquet 1998).

The testing of numerics involves measuring SFs and comparing them with theoretical predictions which help to establish which part of the spectrum is the inertial range, and which scales are dissipative and driving scales. The inertial range in a simulation is often defined as a range of scales where $-\mathrm{SF}_{3 \|} / l$ is closest to its theoretical value, i.e., where the influence of energy injection from driving and energy dissipation from the viscous term is minimized.

Figure 10 shows several structure functions, compensated by various powers of $l$. The ratio of different structure functions can test turbulence self-similarity. If this ratio is dimensionless, it is supposed to be constant through scales. For the test of self-similarity in Fig. 10 we show the ratio of parallel third order structure function and full third order $\mathrm{SF}, \mathrm{SF}_{3}=\left\langle|\mathbf{v}(\mathbf{r}-\mathbf{l})-\mathbf{v}(\mathbf{r})|^{3}\right\rangle$. Figure 10 shows that hydrodynamic turbulence is rather self-similar at the same time the scaling of the second-order 
structure function in the inertial range is around $l^{0.7}$, i.e., close to the Kolmogorov scaling (see next Sect. 4).

\section{Kolmogorov cascade model}

In hydrodynamic turbulence, a useful starting point is the Kolmogorov model (Kolmogorov 1941) for incompressible turbulence. The incompressible case has constant density so that the energy dissipation can be defined per unit mass and assumed statistically homogeneous as well. This quantity, $\varepsilon$ has units of $\mathrm{cm}^{2} / \mathrm{s}^{3}$ and plays a crucial role in many situations and will be used plenty through this review. The Kolmogorov model assumes that the statistical properties of turbulence are uniquely determined by the amount of energy available in this stationary homogeneous system, i.e., by the $\varepsilon$ alone. Furthermore, it is argued that the energy self-similarly cascades through the series of scales known as the inertial range. Cascade means that the energy is being transferred from one scale to another without dissipation.

The dimensional derivation of Kolmogorov scaling involves noting that the spectrum defined in the previous section has units of $\mathrm{cm}^{3} / \mathrm{s}^{2}$ and the wavenumber has units of $\mathrm{cm}^{-1}$, so that

$$
E(k)=C_{K} \varepsilon^{2 / 3} k^{-5 / 3},
$$

where $C_{K}$ is a dimensionless Kolmogorov constant. From the previous section we also know that the $3 \mathrm{D}$ spectrum $F(k) \sim E(k) k^{-2} \sim k^{-11 / 3}$.

The hand-waving derivation involves introducing "characteristic velocity on scale $l$ " $u_{l}$ and imagining that the energy rate is constant for all scales:

$$
u_{l}^{2} / t_{\text {casc }}=\varepsilon,
$$

where $t_{\text {casc }}$ is the "cascading timescale" the time it takes for nonlinearity to remove energy from scale $l$ and transfer it to smaller scales. It is further assumed that in the hydrodynamic cascade $t_{\text {casc }}$ is a dynamical time on each particular scale, i.e., $t_{\text {casc }} \approx l / u_{l}$, which results in

$$
\begin{aligned}
u_{l}^{3} / l & \sim \varepsilon . \\
u_{l} & \sim(\varepsilon l)^{1 / 3} \sim \varepsilon^{1 / 3} k^{-1 / 3} .
\end{aligned}
$$

From the definition of the spectrum and its relation to the SF, we can argue that $E(k) k \sim u_{l}^{2}$ so that the two formula for Kolmogorov scaling agree.

A compilation of experimental results for hydrodynamic turbulence (Sreenivasan 1995 ) suggests that a Kolmogorov constant $C_{K}$ is universal for a wide variety of flows. High-resolution numerical simulations of isotropic incompressible hydrodynamic turbulence, see Fig. 11 and Gotoh et al. (2002), suggest the value around 1.6. See Fig. 12 for a spectrum from $1024^{3}$ simulation from Beresnyak (2011).

The expression Eq. (19) can be written for the largest scale in the system, the outer scale:

$$
\varepsilon=C_{K}^{\prime} \delta v^{3} / L
$$


Fig. 11 Spectra of hydrodynamic turbulence from numerics (solid) and experiments (dots) in dimensionless units. Image reproduced with permission from Gotoh et al. (2002); copyright by AIP
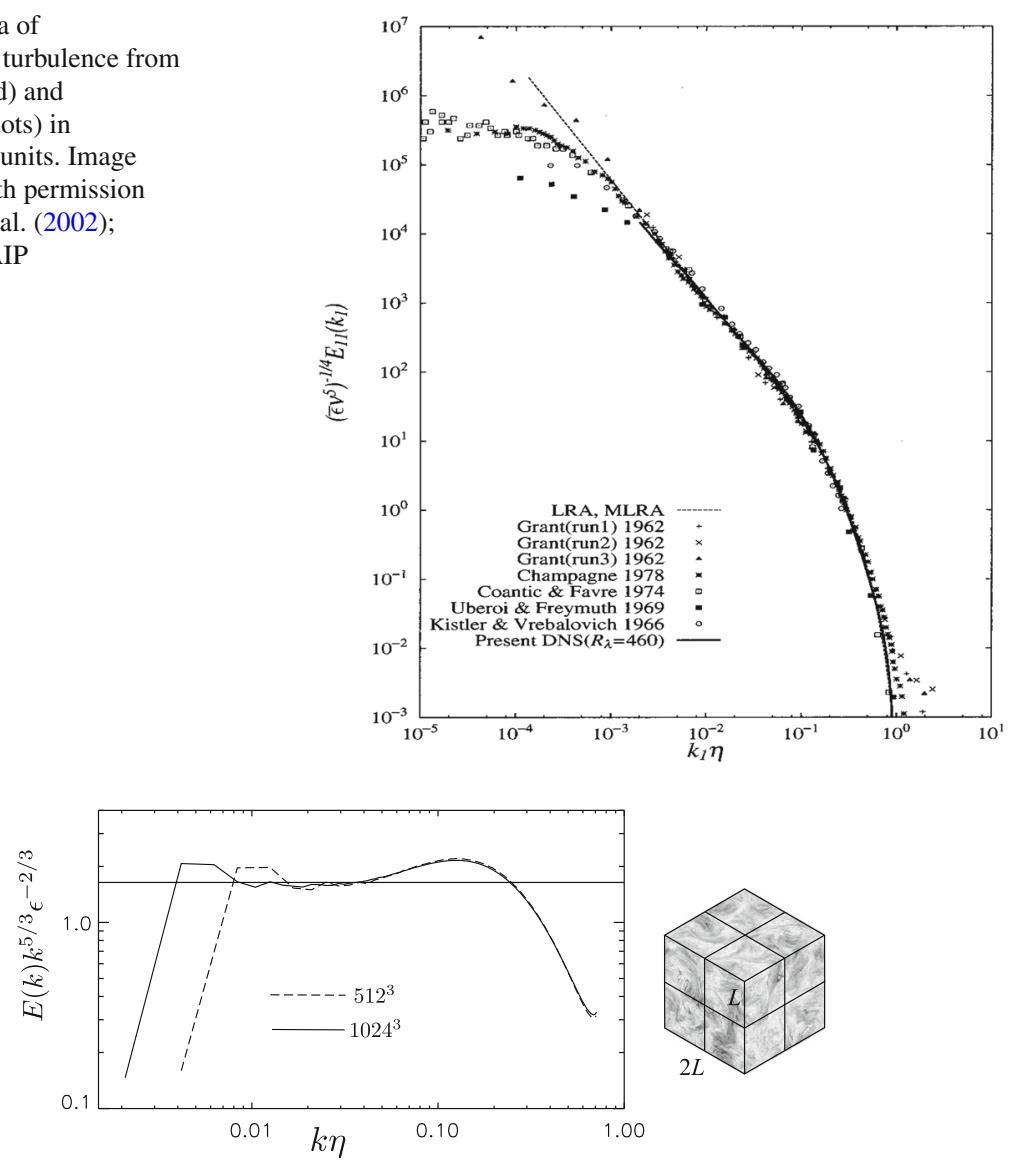

Fig. 12 Spectrum of hydrodynamic turbulence compensated by Kolmogorov scaling to give approximate constant function vs. wavenumber. Statistics from smaller datacube should largely repeat statistics from larger datacubes, as we see on collapsing spectra on the left and visually represented on the right. Image reproduced with permission from Beresnyak and Lazarian (2019); copyright by De Gruyter

This can be regarded as scaling with the outer scale velocity $v$, and/or the scale of the system $L$. Three different things can be done to study this law, known as the zeroth law of turbulence, empirically. One can scale an experimental apparatus from $L$ to $L^{\prime}$, one can increase or decrease velocity, and one can change the fluid to vary viscosity. From the symmetries of the hydrodynamic equations, we know that the only real change would be a change in Reynolds number. The same type of turbulent flow results in approximately the same dimensionless coefficient of $C_{K}^{\prime}$. In the systems that generate turbulence easily, e.g., flow past the grid the above expression is reasonably precise for $\operatorname{Re}>200$. Note that in the statistically stationary case $\varepsilon$ is also the energy dissipation rate, which happens on small scales due to viscosity. This fact illustrates that the outer scale $L$ the dissipative scale only know each other through $\varepsilon$.

Outer scale is sometimes formally defined through integral over the spectrum, e.g., $L=3 \pi / 4 E \int_{0}^{\infty} k^{-1} E(k) d k$. Usually, this is around a scale where energy is 
injected into the system. Inverse cascade of energy in two-dimensional hydrodynamic turbulence is one counter-example of this.

The energy "cascades" down to smaller scales until it hits the so-called Kolmogorov scale, where dissipative processes overcome nonlinear transfer of energy. The Kolmogorov scale can be expressed as a combination of viscosity/diffusivity and energy dissipation rate, which gives a unit of length.

$$
\eta=\left(v_{n}^{3} / \varepsilon\right)^{1 /(3 n-2)},
$$

where $n$ is the order of the viscosity, e.g., $n=2$ for classic molecular viscosity, $v_{n}$ is the value of the diffusivity, so that we obtain Navier-Stokes equation by adding to the RHS of the Euler equation the dissipation operator $-v_{n}\left(-\nabla^{2}\right)^{n / 2}$.

Dimensionless ratio $L / \eta$ could serve as a "length of the inertial range", although in practice spectrum is around an order of magnitude shorter.

Criticism of the Kolmogorov model points to the fact that the assumption of selfsimilarity is quite arbitrary and points to the examples of turbulence which are notably not self-similar. In the three-dimensional hydrodynamic turbulence deviations from self-similarity in the second-order measurements, such as energy spectrum, are fairly small, however. The more precise formula can be obtained by multiplying RHS of Eq. (17) by the "intermittency correction" $(k L)^{\alpha}$, where $\alpha \approx 0.035$. For more details see Frisch (1995).

\subsection{Lagrangian spectrum}

Lagrangian measurements are performed by following a fluid element. Lagrangian viewpoint offers a simpler conceptual picture as the model above is conceptually simpler in Lagrangian formulation. The Euler's equation is a third Newton's law for the fluid element:

$$
\frac{D \mathbf{v}}{D t}=-\frac{\nabla P}{\rho} \text {. }
$$

Here $D / D t$ is the advective (Lagrangian) derivative corresponding to changes of a fluid element's properties over time, $D / D t=\partial / \partial_{t}+\mathbf{v} \cdot \nabla$. The work per unit mass, done upon a fluid element by pressure of surrounding fluid elements will be expressed, therefore as $\mathbf{v} \cdot d \mathbf{v} / d t$. The Kolmogorov theory would therefore assume that, given a characteristic time interval $\tau$, the work done per unit mass upon a fluid element during this interval, $\delta \mathbf{v}_{\tau} \cdot \delta \mathbf{v}_{\tau} / \tau$, will be constant when $\tau$ corresponds to inertial-range timescales and equal to the turbulence energy cascade rate per unit mass $\varepsilon$. Formally, in stationary turbulence the second-order Lagrangian structure function of velocity should satisfy:

$$
\mathrm{SF}(\tau)=\left\langle(\mathbf{v}(t+\tau)-\mathbf{v}(t))^{2}\right\rangle \approx \varepsilon \tau
$$

in the inertial range, where $\mathbf{v}(t)$ is a velocity as a function of time for a given fluid element. This time structure function will correspond to the frequency spectrum of

$$
E(\omega) \approx \varepsilon \omega^{-2}
$$


see Eq. (13). This first appeared in the texbook by Landau and Lifshitz (1959) and also in Corrsin (1963), Tennekes and Lumley (1972). The scaling $\omega^{-2}$ and the fact that the energy spectrum is proportional to energy injection rate $\varepsilon$ appear to be conceptually simpler than $\varepsilon^{2 / 3}$ scaling of the standard Eulerian Kolmogorov scaling.

This spectrum has a dissipation timescale associated with the lifetime of critically damped eddies, also called the Kolmogorov timescale (for $n=2$ ):

$$
\tau_{\eta}=(\nu / \varepsilon)^{1 / 2}
$$

this is the location of the dissipative cutoff in Lagrangian spectrum. The direct measurement of the Lagrangian frequency spectrum is fairly challenging, however, as the probe has to be embedded in the flow. Temporal measurement of spectra from a wind tunnel or channel flow (e.g., Grant et al. 1962) does not correspond to the Lagrangian spectrum but can be connected, by Taylor hypothesis, to spatial spectrum (see Sect. 3). Below, in Sect. 7.3 we explain how a parallel spectrum in MHD turbulence can act as a surrogate of the Lagrangian spectrum.

\subsection{More general Kolmogorov phenomenology}

More general phenomenology is possible assuming cascading time scale relating to the dynamical timescale as

$$
t_{\text {casc }}=\frac{l}{u_{l}}\left(\frac{l}{L}\right)^{-\alpha},
$$

in which case using Eq. (18) we get

$$
u_{l} \sim \varepsilon^{1 / 3} l^{(1-\alpha) / 3} L^{\alpha / 3},
$$

so that, assuming self-similarity, the spectral slope will be $-1-2(1-\alpha) / 3=-5 / 3+$ $2 \alpha / 3$ (Eq. (13)). This will also result in a different Kolmogorov scale which we get by equating cascading time above and the viscous time $l^{n} / v_{n}$ :

$$
\eta_{\alpha}^{3 n-2+2 \alpha}=L^{2 \alpha} \frac{\nu_{n}^{3}}{\varepsilon} .
$$

This reduces to Eq. (22) for $\alpha=0$. Alternatively, one can also assume that interaction is reduced by $u_{l} / c_{s}$ :

$$
t_{\text {casc }}=\frac{l c_{s}}{u_{l}^{2}},
$$

where $c_{s}$ is the sound speed. This model is called acoustic/wave turbulence and gives the $-3 / 2$ spectral slope.

\subsection{Scaling convergence in turbulence: numerics and experiments}

Inertial range in the $3 \mathrm{D}$ numerics is not as big as in nature. In numerics, we use a rigorous quantitative argument to elucidate asymptotic inertial-range scaling. Imagine 
we performed several simulations with different Reynolds numbers. If we believe that turbulence is universal, and the separation of scales between forcing scale and dissipation scale is large enough, the properties of small scales should not depend on how turbulence was driven and also on the scale separation itself. This is because MHD or hydrodynamic equations do not explicitly contain any designated scale, so the simulation with a smaller dissipation scale could be considered, because of the symmetry from equations, as a simulation with the same dissipation scale, but larger driving scale. For example, the small-scale statistics in a $1024^{3}$ simulation will look similar to small-scale statistics in $512^{3}$ simulation, if we keep physical sizes of the grid cell and the dissipation scale the same as in Fig. 12. Another example is the convergence of experimental data as well as numerics onto the same curve in Fig. 11. Note how $\mathrm{x}$ - and $\mathrm{y}$-axis units were made dimensionless using the Kolmogorov length scale and the Kolmogorov velocity scale.

Scaling convergence can be used to compare numerics with measurements, in which can numerics should faithfully reproduce dynamics on all relevant scales, including dissipation scales, e.g., numerics should be "well resolved". However, in the case when several numerical experiments with different Re are compared using scaling convergence, this condition can be somewhat relaxed. Instead, the condition is that discretized formulation works similarly in the compared experiments. The discretization error and other numerical inaccuracies of statistically averaged quantities should depend only on the ratio of Kolmogorov scale to the grid scale provided that the timestep is also determined by the grid scale. So we need to keep the grid scale as the fixed fraction of the Kolmogorov scale. Keep in mind, that Kolmogorov scale itself is determined based on particular phenomenology of the cascade (see Sect. 4.2) and may not be known apriori. In this case, rigorous scaling convergence would require going through available hypotheses and checking each in turn.

We express the spectra of several simulations in dimensionless units corresponding to the expected scaling, for example, a $E(k) k^{5 / 3} \varepsilon^{-2 / 3}$ for the Kolmogorov model and plot it versus dimensionless wavenumber $k \eta$, where dissipation scale $\eta$ again, corresponds to the same phenomenology. On the plot, the two spectra should collapse onto the same curve on the viscous scales, as long as the model works. The method has been used extensively in hydrodynamics (Yeung and Zhou 1997; Gotoh et al. 2002; Kaneda et al. 2003) with great success. In numerics, it is especially efficient since, while experimental data may suffer from systematic uncertainties, numerics does not, and it collects tremendously large statistics on small scales, driving statistical error virtually to zero. Let us understand why this is the case. If we refer to the Kolmogorov cascade picture, described above, the energy cascade is local in scale and the only information that is being transferred from large scales to small scales is the local cascade rate $\varepsilon$. Now, assuming that at each scale, each eddy is independently created, its energy content on this scale should only depend on $\varepsilon$ as $\varepsilon^{2 / 3}$. So if we normalize the measurement by $\varepsilon^{-2 / 3}$, each eddy will represent, presumably, independent estimate of such normalized energy content at each scale. Given a characteristic eddy scale $l$, the number of eddies in a datacube goes as $l^{-3}$, while the number of correlation timescales for strong turbulence goes as $l^{-2 / 3}$, so the statistical error due to volume and time-averaging should decrease as $l^{-11 / 6}$. The plotted normalized spectrum $I(x)=I(k \eta)=E(k) \varepsilon^{-2 / 3} k^{5 / 3}$ should be "pinned" on the dissipation scale, because it should satisfy 


$$
\int_{0}^{\infty} I(x) x^{1 / 3} d x=\frac{1}{2}
$$

The precision of the convergence method was demonstrated in Kaneda et al. (2003), where $4096^{3}$ simulations allowed to capture the intermittency correction, which is a correction of -0.04 to the $-5 / 3$ spectral slope.

\section{MHD equations, modes}

Below we write ideal MHD equations that describe perfectly conducting, inviscid fluid. It should be kept in mind that solving ideal equations often require including special treatment of shocks and turbulence.

$$
\begin{gathered}
\partial_{t} \rho+\nabla \cdot(\rho \mathbf{v})=0, \\
\rho\left(\partial_{t}+\mathbf{v} \cdot \nabla\right) \mathbf{v}=-\nabla P+\mathbf{j} \times \mathbf{B}, \\
\nabla \cdot B=0, \\
\partial_{t} \mathbf{B}=\nabla \times(\mathbf{v} \times \mathbf{B}), \\
P=P(\rho, s)
\end{gathered}
$$

with current $\mathbf{j}=\nabla \times B$ and vorticity $\omega=\nabla \times v, P(\rho, s)$ is an equation of state.

In the ideal case, specific entropy $s$ is decoupled from the rest of the equations and can be described as a passive scalar. Here we use Heaviside units, redefining electric charge with a factor of $1 / 4 \pi$ and getting rid of $4 \pi$ factors in Maxwell's equations.

Introducing sound speed $c_{s}^{2}=\partial P / \partial \rho$, linearized MHD equations reveal four perturbation modes:

(1) Alfvén mode-transverse waves with $\mathrm{v}$ and $\mathrm{B}$ perturbations along $\mathbf{k} \times \mathbf{B}$ and dispersion relation $\omega=\left(\mathbf{v}_{\mathbf{A}} \cdot \mathbf{k}\right)$, where $\mathbf{v}_{\mathbf{A}}=\mathbf{B} / \sqrt{4 \pi \rho}$, so-called Alfvén velocity = magnetic field $\mathbf{B}$ in velocity units introduced earlier. The phase velocity of Alfvén mode is

$$
u_{A}=\omega / k= \pm\left(\mathbf{v}_{\mathbf{A}} \cdot \hat{\mathbf{k}}\right)= \pm v_{A} \cos \theta
$$

while its group velocity $\partial \omega / \partial \mathbf{k}= \pm \mathbf{v}_{\mathbf{A}}$, hence the term Alfvén velocity.

$(2,3)$ Fast and slow modes-compressible waves with perturbations in the $\mathbf{k}, \mathbf{B}$ plane propagating correspondingly faster and slower than $v_{A}$, with the dispersion relation

$$
u_{f, s}^{2}=\omega^{2} / k^{2}=\frac{1}{2}\left[\left(v_{A}^{2}+c_{S}^{2}\right) \pm \sqrt{\left(v_{A}^{2}+c_{S}^{2}\right)^{2}-4 v_{A}^{2} c_{S}^{2} \cos ^{2} \theta}\right] .
$$

(4) Entropy mode-non-propagating passive scalar perturbations of specific entropy (Landau and Lifshitz 1960; Biskamp 2003).

In this section, we will skip eigenvectors for the three modes for brevity and write them out in Sect. 10. 


\section{Numerical methods to simulate MHD turbulence}

Several tools are available to simulate turbulence numerically:

\subsection{Pseudospectral codes}

The pseudospectral code solves MHD equations as a set of ordinary differential equations in time for each spacial Fourier harmonic. The coupling of harmonics is through the nonlinear term which is calculated in real space (hence "pseudo") and then converted back to Fourier space. Since the pseudospectral method approximates derivatives non-locally, using all data points, it does not suffer from dispersion error. Also, if some care is taken with timestep integration, for example, a symplectic integrator is used, it also preserves energy, i.e., does not suffer from dissipation error. The explicit dissipation, e.g., viscosity or resistivity are done with simple algebraic operations in Fourier space and can be made unconditionally stable, irrespective of the time step with no numerical expense. A typical pseudospectral code have symplectic integrator, corrects for aliasing error and has explicit dissipation in the form $a_{t+\Delta t}=$ $a_{t} \exp \left(-v k^{2} \Delta t\right)$. Aliasing error comes from frequencies above $2 / 3$ or below $-2 / 3$ of the Nyquist frequency if the nonlinear term is second order. For example, if the Nyquist frequency is $\pi$, and keeping frequencies within $[-2 / 3 \pi, 2 / 3 \pi]$, the sum or difference will still be within the interval modulo $2 \pi$ (e.g., $2 / 3 \pi+2 / 3 \pi-2 \pi=-2 / 3 \pi$ ). Another advantage of pseudospectral code for the incompressible case is that divergence-free condition for velocity and magnetic field can be done with simple algebraic operations in Fourier space. The spacial reconstruction uses all Fourier harmonics. As a result, the method's precision increases exponentially with the number of points in one dimension. This makes it practical to do "fully resolved" simulations, i.e., when the viscosity and magnetic diffusivities are explicit, and all scales of interests are represented with reasonable precision. The usual rule of thumb for well-resolved simulation in a periodic box with size $2 \pi$ and number of points $N$ in one direction, when the wavenumbers are represented by integers $[-N / 2+1, \ldots, 0,1, \ldots, N / 2]$ is $k_{\max } \eta \gtrsim 1$, where $k_{\max }=N / 3$ for a $2 / 3$ dealiased code. The main disadvantage is difficulty in introducing arbitrary boundary conditions. This method's periodic box comes naturally when we try to simulate homogeneous isotropic turbulence, however. One example of a publicly available pseudospectral code is SNOOPY: http://ipag.osug.fr/ lesurg/snoopy.html.

\subsection{Finite difference codes}

Finite difference codes estimate derivatives by finite differencing. The precision of the code increases, typically, as a power law with the number of points, the index of the power law is the "order" of the code. The main advantage is simplicity and numerical speed. Disadvantages include special treatment of shocks with "shock viscosity". High order finite difference codes with explicit diffusivities can be rather precise in simulating turbulence. The divergence-free condition can be kept with "divergence cleaning" or with equations formulated in terms of magnetic potential. The PENCIL code is a popular, publicly avalable, high-order finite-difference code: https://github. com/pencil-code. 


\subsection{Finite volume codes with Riemann solvers}

Also known as Godunov codes. Finite volume codes keep values of cell averages then reconstruct (interpolate) values on the interface of the cells both from the right and from the left. Thus the interface value is discontinuous and may be evolved for a short time as a "Riemann problem"-initial value problem with a single discontinuity. The time-average fluxes of conserved quantities through the interface are then computed from, typically, the approximate solution of the Riemann problem by the "Riemann solver". Finally, fluxes are used to advance cell averages in time. The inherent ability to describe discontinuities makes Godunov codes very robust and a code of choice to simulate supersonic turbulence. See also documentation of the publicly available code ATHENA++: https://github.com/PrincetonUniversity/athena-public-version.

\subsection{Lagrangian codes}

Lagrangian codes refer to codes that use grid or material elements that are moving with the fluid. These include N-body codes (e.g., collisionless particles moving under the action of gravity), smooth particle hydrodynamics (SPH) codes-particle codes simulating hydrodynamics, moving mesh codes, among which purely Lagrangian (mesh moving with the fluid) or arbitrary Lagrangian-Eulerian (ALE, mesh moving in an arbitrary way). These codes are often used to simulate collapse under gravitational forces, but less common to simulate turbulence.

A publicly avalable code is GADGET2: https://wwwmpa.mpa-garching.mpg.de/ gadget/.

\section{Theory of Alfvénic turbulence}

In Sect. 4, we introduced the standard Kolmogorov description of the inertial range of incompressible hydrodynamic turbulence. It is clear, however, that this picture is not applicable to MHD. Turbulence spectra are typically steeper than $k^{-1}$ meaning that RMS fields are dominated by large scales. In hydrodynamics, however, large-scale velocity can be nullified by an appropriate choice of the reference frame. In MHD largescale magnetic field cannot be nullified and will be dynamically important on all scales, including very small scales. This combination of sizable RMS large-scale field and small-scale fluctuations of the fields is the main difference from hydrodynamics. Also known as a "strong field limit", it was pointed out by Iroshnikov (1964), Kraichnan (1965) and it was suggested that inertial-range MHD turbulence is weak turbulence. Here weak turbulence refers to the picture of wave turbulence where wave packets propagate almost freely, and collision between waves leads to the small perturbation in their structure so that the perturbation theory is applicable (Zakharov et al. 1992). The interaction of wave packets in MHD, however, is very different from the collision of sound waves. Introducing wavevector components parallel and perpendicular to the mean field, $k_{\|}$and $k_{\perp}$ we see that the wave frequency $\omega=k_{\|} v_{A}$ depends only on $k_{\|}$. This anisotropic dispersion relation results in anisotropic turbulence. 
The subsequent analytic work demonstrated that MHD turbulence tends to become stronger and not weaker during the cascade (Galtier et al. 2000), as we will show below. We will also show that the Alfvénic part of MHD perturbations governs this highly anisotropic turbulence, hence the term "Alfvénic turbulence".

The rationale of working with simplified incompressible equations is similar to hydrodynamics. Assuming that (a) turbulence has no shocks, (b) no sizable energy is carried by sound waves (in MHD case, fast MHD mode), (c) the Mach number $M_{s}=V_{L} / c_{s}$ is small, we can argue that a scale-wise Mach number $M_{s}=\delta v / c_{s}$ should also be small and decrease with scale. The fluid compressibility will, therefore, be small in the inertial range.

Incompressible MHD equations consist of two dynamical equations and two constraints:

$$
\begin{gathered}
\partial_{t} \mathbf{v}=-\nabla P^{\prime} / \rho-(\nabla \times \mathbf{v}) \times \mathbf{v}+(\nabla \times \mathbf{b}) \times \mathbf{b}, \\
\partial_{t} \mathbf{b}=\nabla \times(\mathbf{v} \times \mathbf{b}), \\
\nabla \cdot v=0, \\
\nabla \cdot b=0 .
\end{gathered}
$$

Here we normalized magnetic field to velocity units in the same manner as in previous sections, i.e., $\mathbf{b}=\mathbf{v}_{\mathbf{A}}=\mathbf{B} / \sqrt{4 \pi \rho}$. The dynamical equations are known as the momentum equation and the induction equation. The induction equation honors the divergence-free constraint for the magnetic field, this effectively results in no new constraint, if the initial condition is chosen divergence-free. The divergence-free constraint for velocity is satisfied by the appropriate choice of the scalar function $P^{\prime}=P+\rho v^{2} / 2$. The pressure, therefore, is a dummy variable.

Introducing solenoidal projection $\hat{S}=\left(1-\nabla \Delta^{-1} \nabla\right)$ we can rewrite the equations without explicit constraints:

$$
\begin{gathered}
\partial_{t} \mathbf{v}=\hat{S}(-(\nabla \times \mathbf{v}) \times \mathbf{v}+(\nabla \times \mathbf{b}) \times \mathbf{b}), \\
\partial_{t} \mathbf{b}=\nabla \times(\mathbf{v} \times \mathbf{b}) .
\end{gathered}
$$

Very useful change of variables to the Elsässer variables $\mathbf{w}^{ \pm}=\mathbf{v} \pm \mathbf{b}$ makes these equations even more compact:

$$
\partial_{t} \mathbf{w}^{ \pm}+\hat{S}\left(\mathbf{w}^{\mp} \cdot \nabla\right) \mathbf{w}^{ \pm}=0 .
$$

We introduce total energy, $1 / 2 \int\left(v^{2}+b^{2}\right) d \mathbf{r}$ and cross-helicity $\int \mathbf{v} \cdot \mathbf{b} d \mathbf{r}$ which are conserved in this incompressible formulation. By taking the sum and difference of these quantities, we obtain conservation of each of Elsässer energies $1 / 2 \int\left(w^{ \pm}\right)^{2} d \mathbf{r}$.

\subsection{From weak to strong turbulence}

Keeping in mind the above argument of a sizable mean field let us explicitly write it down as the constant field $\mathbf{v}_{\mathbf{A}}$ in a given volume, and perturbations as $\delta \mathbf{w}^{ \pm}=\mathbf{w} \pm \mathbf{v}_{A}$ :

$$
\partial_{t} \delta \mathbf{w}^{ \pm} \mp\left(\mathbf{v}_{\mathbf{A}} \cdot \nabla\right) \delta \mathbf{w}^{ \pm}+\hat{S}\left(\delta \mathbf{w}^{\mp} \cdot \nabla\right) \delta \mathbf{w}^{ \pm}=0 .
$$


Let us denote $\|$ and $\perp$ as directions parallel and perpendicular to $\mathbf{v}_{A}$ and the subscript to the vector means projection to $\mathbf{v}_{A}$ or the perpendicular plane, respectively.

In the limit of small $\delta w$ 's they represent perturbations, propagating along $\mathbf{B}$ or in the opposite direction, with the nonlinear term describing their interaction. Note that "self-interaction" of $\delta w^{+}$or $\delta w^{-}$is absent, both being an exact solution in the absence of another. The dominant nonlinear interaction is a three-wave process, so writing the dispersion relation and the conservation laws for energy and momentum,

$$
\begin{aligned}
\omega_{n} & =k_{\| n} v_{A}, \\
\pm \omega_{1} & = \pm \omega_{2} \pm \omega_{3}, \\
k_{\| 1} & =k_{\| 2}+k_{\| 3}, \\
k_{\perp 1} & =k_{\perp 2}+k_{\perp 3}
\end{aligned}
$$

we see that one of the $\omega_{n}$ must be zero. Let us choose $\omega_{3}=0$, this means $\left|k_{\| 1}\right|=\left|k_{\| 2}\right|$, but there is no restrictions on $k_{\perp 1,2}$. The cascade preserves frequencies and goes forward by increasing only $k_{\perp}$.

In wave turbulence theory the interaction strength $\xi$ is the ratio of the nonlinear shear rate $k_{\perp} \delta w$ to the wave frequency $k_{\|} v_{A}$, it describes a fractional perturbation during one wave period:

$$
\xi=k_{\perp} \delta w / k_{\|} v_{A} .
$$

It is also the estimate of the ratio of the nonlinear term to the mean-field term in Eq. (46). In MHD turbulence the dynamical timescale $\tau_{\text {dyn }}=1 / k_{\perp} \delta w$ does not have to be proportional to the cascade timescale as in hydrodynamic turbulence. Instead, $\tau_{\text {casc }}$ is increased by a factor of $1 / \xi$. This can also be understood in terms of perturbations of a wave packet being a random walk. Each individual perturbation is $\xi$ strong, so it takes $(1 / \xi)^{2}$ steps to destroy the wavepacket completely:

$$
\tau_{\text {casc }}=\left(1 / k_{\|} v_{A}\right)(1 / \xi)^{2}=k_{\|} v_{A} /\left(k_{\perp} \delta w\right)^{2} .
$$

The energy cascade rate is the energy on each scale divided by the cascade time on this scale. This rate is expected to be constant through scales and we designate it $\varepsilon$ :

$$
\delta w^{2} \frac{\left(\delta w k_{\perp}\right)^{2}}{v_{A} k_{\|}}=\varepsilon .
$$

Note $k_{\|}$here is constant, so the phenomenological cascade spectrum is determined by $\delta w^{2} \sim k_{\perp}^{-1}$, which corresponds to one-dimensional perpendicular spectrum $E\left(k_{\perp}\right) \sim \delta w^{2} k_{\perp}^{-1} \sim k_{\perp}^{-2}$. This argument can be followed rigorously by perturbation collision integral approach, used in wave turbulence (Zakharov et al. 1992) and solved exactly by Zakharov transformation, which was accomplished in Galtier et al. (2000, 2002).

One consequence of this solution is that turbulence grows anisotropic, with $k_{\perp} / k_{\|} \sim$ $k_{\perp}$. Interestingly, it becomes stronger and not weaker on smaller scales, in other words, $\xi$ is an increasing function of $k_{\perp}$. Indeed, if we maintain $k_{\|}$constant, this will result in 


$$
\xi=\frac{\delta w k_{\perp}}{v_{A} k_{\|}} \sim k_{\perp}^{1 / 2} \underset{k_{\perp} \rightarrow \infty}{\longrightarrow} \infty .
$$

Our two conclusions from this simple perturbation theory is that: (a) the resonance condition results in a "perpendicular cascade", making MHD turbulence anisotropic, (b) turbulence becomes stronger along the cascade until $\xi \sim 1$.

One can wonder if weak MHD turbulence is ever realized in nature. We can hypothesize that this is the case in astrophysical objects where the strong magnetic field is anchored in a heavy object, i.e., a star and is extended into the magnetosphere where perturbations of the field are much smaller than this anchored field. The empirical evidence for this case and specifically for the $k^{-2}$ perpendicular spectrum is lacking, however. One can argue that large-scale dynamo (which we consider in subsequent sections) can generate a mean field which is much stronger than perturbations, but empirically we know from the ISM observations that they are of the same order. This results in MHD turbulence being strong on the outer scale.

\subsection{Reduced MHD approximation}

Equation (46) can be further simplified assuming anisotropy $k_{\perp} \gg k_{\|}$and the fact that $\delta w \ll v_{A}$. This allows to neglect parallel gradients in the nonlinear term, indeed, the mean field term with the parallel gradient $\left(v_{A} \nabla_{\|}\right) \delta w^{ \pm}$is always much larger than similar contribution from the nonlinear term, $\left(\delta w_{\|}^{\mp} \nabla_{\|}\right) \delta w^{ \pm}$and the latter could be ignored. So the three vector components of Eq. (46) are split into interdependent equations for the scalar $\delta w_{\|}^{ \pm}$and vector $\delta \mathbf{w}_{\perp}^{ \pm}$:

$$
\begin{aligned}
& \partial_{t} \delta \mathbf{w}_{\|}^{ \pm} \mp\left(\mathbf{v}_{\mathbf{A}} \cdot \nabla_{\|}\right) \delta \mathbf{w}_{\|}^{ \pm}+\hat{S}\left(\delta \mathbf{w}_{\perp}^{\mp} \cdot \nabla_{\perp}\right) \delta \mathbf{w}_{\|}^{ \pm}=0, \\
& \partial_{t} \delta \mathbf{w}_{\perp}^{ \pm} \mp\left(\mathbf{v}_{\mathbf{A}} \cdot \nabla_{\|}\right) \delta \mathbf{w}_{\perp}^{ \pm}+\hat{S}\left(\delta \mathbf{w}_{\perp}^{\mp} \cdot \nabla_{\perp}\right) \delta \mathbf{w}_{\perp}^{ \pm}=0,
\end{aligned}
$$

Note that Eq. (55) depends on Eq. (56), but not vice-versa. Since Eq. (55) represent passive dynamics and does not have essential nonlinearity, the nonlinear cascade is completely governed by Eq. (56). This latter equation is known as reduced MHD. In this anisotropic limit, the $\delta \mathbf{w}_{\perp}^{ \pm}$is purely the Alfvén mode, and $\delta w_{\|}^{ \pm}$is the amplitude of the slow mode. Turbulence in Eq. (56) is called Alfvénic turbulence.

Slow mode for $\delta w_{\|}^{+}$is a passive scalar to $\delta \mathbf{w}_{\perp}^{-}$and vice versa. If Alfvén and slow modes will be injected similarly from large scales, they will have the same statistics. In practice, the slow mode content can be determined from numerics.

It turns out, reduced MHD is more general than incompressible MHD and can be used beyond collisional fluid description. Alfvénic perturbations are transverse and rely only on the tension of the magnetic field line as a restoring force, the charged particles tied to this magnetic field line provide inertia. The $[\mathbf{E} \times \mathbf{B}]$ drift waves with wavelengths much smaller than the ion skin depth are indeed just Alfvén waves, and they exist regardless of the collisionality of the plasma (Schekochihin et al. 2009), which is useful for the description of the collisionless solar wind. The anisotropy of MHD turbulence has been known empirically for a while, and RMHD had been formulated for perturbations in plasma in strongly magnetized case some time ago 
(Kadomtsev and Pogutse 1974; Strauss 1976). Since RMHD motions do not require plasma pressure we assume the results that we find for Alfvénic turbulence in this section do not depend on the ratio of plasma pressure to magnetic pressure " $\beta$ ", despite we started the derivation assuming infinite $\beta$.

Introducing parallel length $\Lambda=2 \pi / k_{\|}$and perpendicular length $\lambda=2 \pi / k_{\perp}$ we see that reduced MHD has a two-parametric symmetry:

$$
\mathbf{w} \rightarrow \mathbf{w} A, \lambda \rightarrow \lambda B, t \rightarrow t B / A, \Lambda \rightarrow \Lambda B / A
$$

$A$ and $B$ are arbitrary parameters of the transformation. This is the same symmetry as in hydrodynamics, except for the parallel scale $\Lambda$ transforms similar to time, not to length. $\Lambda$ being similar to time is very important and leads to analogies between dynamics in time and parallel structure in space as we show below. MHD equations do not have such symmetry, so Kolmogorov self-similarity arguments, technically, can not be applied to the MHD case. In practice, this regime for MHD can be achieved within the inertial range where $\delta w \ll v_{A}$ condition and anisotropy condition are satisfied. In numerics, it is challenging to reach these universal dynamics directly from isotropic scales with $\delta w \sim v_{A}$. Instead, one can directly solve RMHD equations. As a practical comment, the statistics from the full MHD with $\delta w^{ \pm} \sim 0.1 v_{A}$ is very close of that one of RMHD, see Beresnyak and Lazarian (2009b).

Another symmetry is evident in RMHD, related to the value of $v_{A}$, The equations are unchanged under transformation $v_{A} \rightarrow v_{A} A, \Lambda \rightarrow \Lambda A$. The parallel scale and the Alfvén speed can be rescaled simultaneously without changing the dynamics.

\subsection{Strong MHD turbulence}

As we demonstrated above in Sect. 7.1, the perpendicular cascade will result in the growth of $\xi$ and will naturally lead to strong turbulence, with $\xi \sim 1$. Goldreich and Sridhar (1995) proposed that the growth of $\xi$ will be limited by the uncertainty relation between the cascading timescale and the wave-packet frequency, namely that the cascade time cannot be shorter than the wave period: $\tau_{\text {casc }} \omega \geq 1$. Using Eq. (52) we get $\xi \leq 1$. This will make $\xi$ to be stuck around unity, which was termed as "critical balance" by Goldreich and Sridhar. As far as $\xi \sim 1$ we have $\tau_{\text {dyn }} \sim \tau_{\text {casc }} \sim 1 / \omega$, we can regard turbulence as "strong" and apply Kolmogorov phenomenology. For the cascade of the two Elsässer energies:

$$
\varepsilon^{ \pm}=\frac{\left(\delta w_{\lambda}^{ \pm}\right)^{2} \delta w_{\lambda}^{\mp}}{\lambda},
$$

These are two independent cascades, but in a theory with $\varepsilon^{+}=\varepsilon^{-}$this becomes standard Kolmogorov phenomenology in the $k_{\perp}$ direction.

$$
E(k)=C_{K} \varepsilon^{2 / 3} k_{\perp}^{-5 / 3},
$$

We will return to the more general "imbalanced" case with $\varepsilon^{+} \neq \varepsilon^{-}$in the next section, but briefly note that such theory is non-trivial since it is impossible to maintain critical balance for resonant waves with different amplitude. 
The assumption of critical balance $\xi \sim 1$ allows us to estimate perturbation anisotropy directly. The "wavevector anisotropy" relates two wavevectors at which the one-dimensional spectrum along the field and perpendicular to the field have the same power. A similar relation can be obtained between parallel and perpendicular scales $\Lambda$ and $\lambda$ vis SFs. Using $\xi=1$, and the $-5 / 3$ scaling $\delta w \sim \lambda^{1 / 3}$ we obtain $k_{\|} \sim k_{\perp}^{2 / 3}$, which is known as GS95 anisotropy.

There is a different argument, however, that is sufficient to obtain this anisotropy. This argument is based on RMHD symetry $\Lambda \sim v_{A}$ we discussed in Sect. 7.2 and dimensional grounds. Indeed, if we have $\Lambda \sim v_{A}$, the rest of the expression for $\Lambda$ must have units of time, which is uniquely obtained from $\lambda$ and $\varepsilon$ as $\lambda^{2 / 3} \varepsilon^{-1 / 3}$ :

$$
\Lambda=C_{A} v_{A} \lambda^{2 / 3} \varepsilon^{-1 / 3},
$$

where we introduced a dimensionless "anisotropy constant" $C_{A}$.

The perpendicular SF which correspond to $k_{\perp}^{-5 / 3}$ spectrum will have the scaling $\mathrm{SF}_{\perp} \sim \lambda^{2 / 3}$ (Eq. (13)), while inserting $\Lambda \sim \lambda^{2 / 3}$, we get parallel structure function as $\mathrm{SF}_{\|} \sim \lambda^{2 / 3} \sim \Lambda$.

The parallel spectrum, which corresponds to such SF is $E\left(k_{\|}\right) \sim k_{\|}^{-2}$ and from dimensional arguments we recover the prefactor as

$$
E\left(k_{\|}\right)=C_{\|} \varepsilon v_{A}^{-1} k_{\|}^{-2},
$$

where we introduced dimensionless constant $C_{\|}$.

Equations (59) and (60) (or, alternatively (61)) describe the spectrum and anisotropy of MHD turbulence, which may still be corrected for intermittency.

A modification which leads to a shallower and not steeper spectrum was proposed in Boldyrev (2005, 2006), henceforth B06 suggesting that GS95 scalings are modified by a scale-dependent factor that decreases the strength of the interaction, effectively, the theory described in Sect. 4.2 with $\alpha=1 / 4$. Different arguments to the same effect were proposed in Gogoberidze (2007). In this case the spectrum will be expressed as $E(k)=C_{K 2} \varepsilon^{2 / 3} k^{-3 / 2} L^{1 / 6}$, see Eq. (28), the factor $\xi$ is modified by $(l / L)^{1 / 4}$, so that anisotropy follows modified critical balance with $k_{\|} \sim k_{\perp}^{1} / 2$. The Kolmogorov scale of B06 model is obtained from Eq. (29): $\eta_{1 / 4}=\left(v_{n}^{3} / \varepsilon\right)^{1 /(3 n-1.5)} L^{0.5 /(3 n-1.5)}$.

It turns out the anisotropy can be argued from the Lagrangian frequency spectrum without postulating critical balance or involving uncertainty relations. In the incompressible MHD all modes propagate with the same speed, the Elsässer components propagate either along or against the local magnetic direction, i.e., along with the magnetic field line. This propagation will be described by the functional form $f\left(s \mp v_{A} t\right)$, where $s$ is a distance along the field line. The nonlinear interaction will contribute to the slower time evolution of $f$ and the trajectory $s= \pm v_{A} t$ will be analogous to following hydrodynamic fluid element in the Lagrangian formulation. Let us record $\mathbf{w}^{+}$ and $\mathbf{w}^{-}$along the field line in a fixed time. The positive direction $s$ will be equivalent to following the evolution of $\mathbf{w}^{+}$backward in time and $\mathbf{w}^{-}$forward in time. In measuring the frequency spectrum, the sign of time will be unimportant. So the measurement of power spectrum along the field line will be analogous to Lagrangian frequency spectrum with frequency $\omega$ replaced by the wavenumber $k_{\|}=\omega / v_{A}$ (Beresnyak 2014): 
Table 1 Three-dimensional MHD and RMHD simulations. Table reproduced with permission from Beresnyak (2015); copyright by AAS

\begin{tabular}{llllllll}
\hline Run & $N^{3}$ & Dissipation & $v_{A}$ & $\varepsilon$ & $\eta$ & $k_{\max } \eta$ & $v_{A} \tau_{\eta}$ \\
\hline MHD1 & $1536^{3}$ & $-5 \cdot 10^{-10} k^{4}$ & 0.73 & 0.091 & 0.0021 & 1.08 & 0.026 \\
MHD2 & $1536^{3}$ & $-6.2 \cdot 10^{-10} k^{4}$ & 1.53 & 0.728 & 0.0018 & 0.92 & 0.025 \\
M1 & $1024^{3}$ & $-1.75 \cdot 10^{-4} k^{2}$ & 1 & 0.06 & 0.0031 & 1.05 & 0.044 \\
M2 & $2048^{3}$ & $-7 \cdot 10^{-5} k^{2}$ & 1 & 0.06 & 0.00155 & 1.06 & 0.028 \\
M3 & $4096^{3}$ & $-2.78 \cdot 10^{-5} k^{2}$ & 1 & 0.06 & 0.00077 & 1.06 & 0.017 \\
M1H & $1024^{3}$ & $-1.6 \cdot 10^{-9} k^{4}$ & 1 & 0.06 & 0.0030 & 1.04 & 0.045 \\
M2H & $2048^{3}$ & $-1.6 \cdot 10^{-10} k^{4}$ & 1 & 0.06 & 0.00152 & 1.04 & 0.029 \\
M3H & $4096^{3}$ & $-1.6 \cdot 10^{-11} k^{4}$ & 1 & 0.06 & 0.00076 & 1.04 & 0.018 \\
\hline
\end{tabular}

$$
E\left(k_{\|}\right)=C_{\|} E(\omega) \frac{d \omega}{d k_{\|}}=C_{\|} \varepsilon\left(v_{A} k_{\|}\right)^{-2} v_{A}=C_{\|} \varepsilon v_{A}^{-1} k_{\|}^{-2}
$$

This is the same expression as obtained in Sect. 7.3 from phenomenological considerations. The parallel structure function $\mathrm{SF}_{\|}(l) \sim \varepsilon l v_{A}^{-1}$ The dimensional argument involving Alfvén symmetry of reduced MHD arrive at the same result (Beresnyak 2012b). This symmetry allows $E\left(k_{\|}\right) d k_{\|}$to depend only on $k_{\|} v_{A}$, which will require that $E\left(k_{\|}\right) \sim v_{A}^{-1}$. The rest of the expression can be obtained from units.

\subsection{Numerics: perpendicular spectrum}

Table 1 presents parameters of DNS strong MHD and RMHD turbulence (first presented in Beresnyak 2014, averaged statistics are publicly available at https://sites. google.com/site/andreyberesnyak/simulations/big3.), these are a well-resolved driven statistically stationary simulations intended to precisely calculate averaged quantities. MHD cases labeled MHD1-2 have no mean field, $B_{0}=0$ so that $v_{A}$ is defined only locally with the Table listing RMS values of $v_{A}$. Two series of RMHD driven simulations are described in Table 1 as M1-3 and M1-3H. These have a strong mean field we denote $B_{0}$, RMS fields $v_{\mathrm{rms}} \approx B_{\mathrm{rms}} \approx 1$, perpendicular box size of $2 \pi$ and parallel box size of $2 \pi B_{0}$. The driving was anisotropic with anisotropy $B_{0} / B_{\mathrm{rms}}$ so that turbulence starts being strong from the outer scale. Technically, $B_{0}$ is arbitrary. However, the RMHD limit is only applicable to very large $B_{0}$ as we showed above.

In simulations, we see a rapid decrease of parallel correlation length right after the driving scale, which indicates the efficiency of nonlinear interaction and the regime of strong turbulence. The correlation timescale for $v$ and $B$ was around $\tau \approx 0.97$, so the box contained around 6.5 parallel correlation lengths. Each simulation was started from long-evolved low-resolution simulation and was subsequently evolved for $\Delta t=13.5$ in code units in high resolution, and we used the last 7 dynamical times for averaging. In earlier work (Beresnyak 2011, 2012a) it was found that averaging over $\sim 7$ correlation timescales gives a reasonably good statistic on the outer scale and very good statistics on smaller scales. 


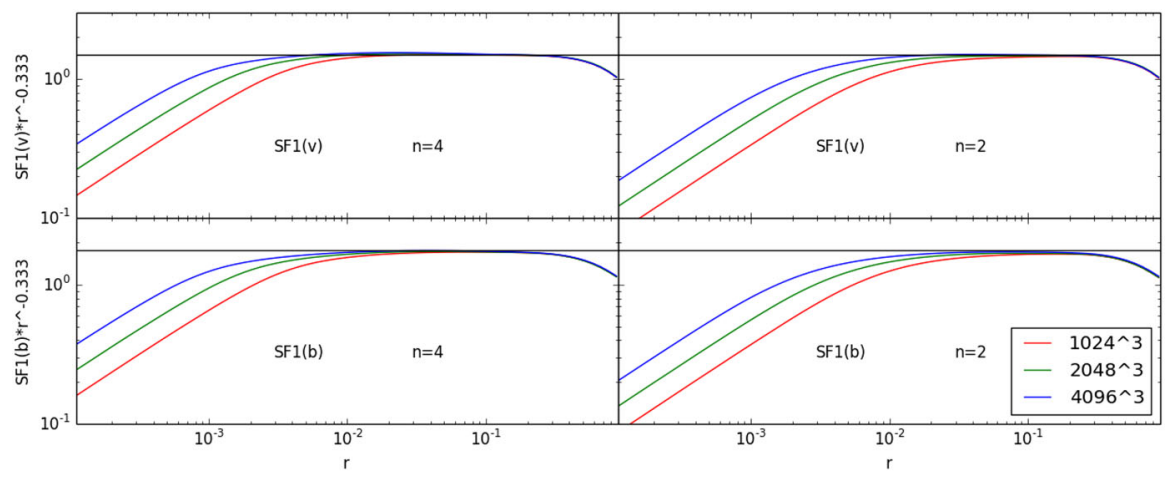

Fig. 13 First order SFs of velocity (top) and magnetic field (bottom) compensated by $r^{1 / 3}$ (Kolmogorov scaling). Left: M1-3H, right: M1-3
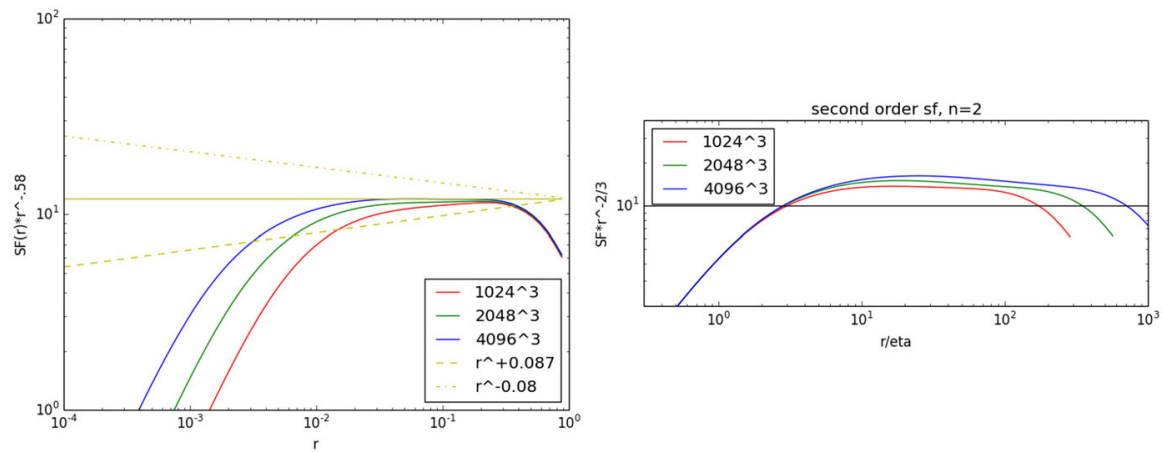

Fig. 14 Second order SF scaling for M1-3. Left: SF plotted vs dimensional distance $r$ compensated by $r^{-0.58}$. Right: scaling convergence study for the $r^{2 / 3}$ (Kolmogorov) scaling, described in Sect. 4.3, both axes are dimensionless. We see convergence, i.e., the overall scaling is $r^{2 / 3}$

Numerically, we used $k_{\max } \eta>1$ resolution criterion, with $\eta$ being classic Kolmogorov scale. Additionally, we checked the precision of the spectra by performing a resolution study on lower resolutions. In particular, we saw spectral error lower than $8 \times 10^{-3}$, up to $k \eta=0.5$ when increasing resolution from $576^{3}$ to $960^{3}$ and the spectral error lower than $3 \times 10^{-3}$ when we increased parallel resolution in a $1152^{3}$ simulation by a factor of two. We presume this error is a mostly systematic error, associated with grid effects because the statistical error is likely to be vanishingly small, see the end of Sect. 4.3. We did not use any data above $k \eta=0.5$ for fitting as the spectrum sharply declines after this point and contains negligible energy.

In Fig. 13 we plotted first order perpendicular structure functions of velocity and magnetic field. These seem to scale with the Kolmogorov power of $r^{1 / 3}$. In Fig. 14 we show second-order SFs for M1-3. On the left of this figure are SFs vs. distance. We see that the scaling is not obvious, with higher-resolution SF having the shallower slope of the flat part, the $4096^{3}$ seemingly having $r^{0.58}$ scaling not expected from theory. On the right of Fig. 14 we use rigorous scaling convergence study (Sect. 4.3) to show that the overall scaling is $r^{2 / 3}$ (Kolmogorov). 


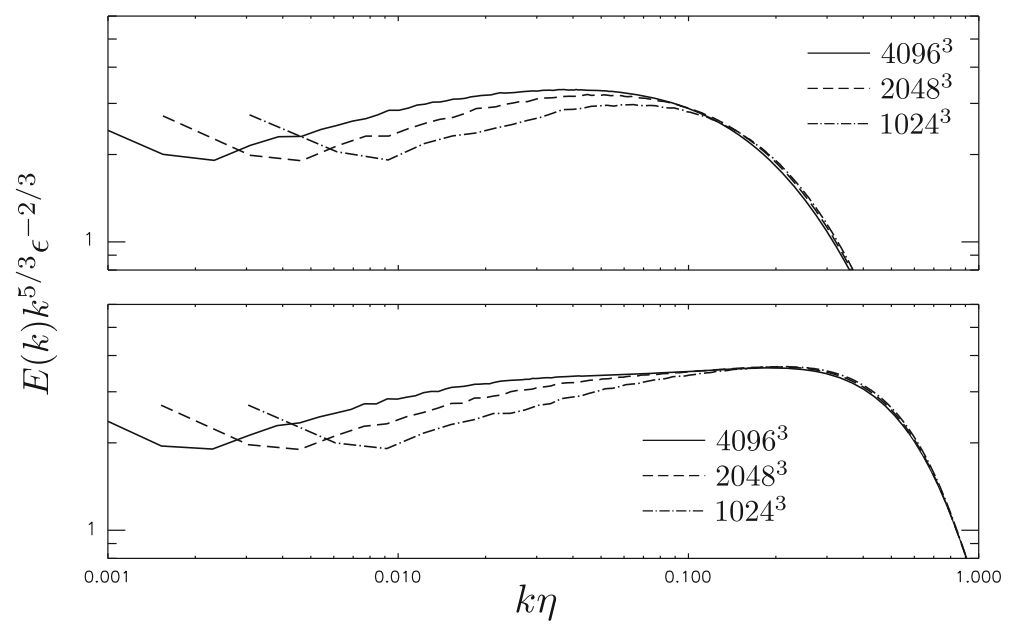

Fig. 15 Checking -5/3 hypothesis with the scaling convergence study (Sect. 4.3) of perpendicular spectrum. Solid, dashed and dash-dotted lines are the spectra from $4096^{3}, 2048^{3}$ and $1024^{3}$ simulation correspondingly. The upper plot shows normal diffusion M1-3 simulations, and the lower plot shows hyperdiffusive $\mathrm{M} 1-3 \mathrm{H}$ simulations. The convergence is reasonable around the dissipation scale. The scaling that achieves the best convergence is $\approx-1.70$. Applying the same method to the $-3 / 2$ slope model results in the lack of convergence. Image reproduced with permission from Beresnyak (2014); copyright by AAS

Figure 15 presents a convergence test of the perpendicular 3D spectrum for the $-5 / 3$ model, and the convergence is reasonable, while the best convergence is reached at the -1.7 scaling. Figure 16 shows a convergence study of the residual energy spectrum (magnetic energy minus kinetic energy). The best convergence is, again, near -1.7 slope. In all cases the convergence is consistent across two simulation groups with different dissipation prescriptions, M1-3 and M1-3H.

The flat part of the normalized spectrum can be used to obtain a Kolmogorov constant of $C_{K A}=3.3 \pm 0.1$, which was first reported in Beresnyak (2011). The total Kolmogorov constant for both Alfvén and slow mode in the above paper was estimated as $C_{K}=4.2 \pm 0.2$ for isotropically driven turbulence with zero mean field. This was obtained using an empirical energy ratio between slow and Alfvén mode, $C_{S}$ which is between 1 and 1.3. This larger value of Kolmogorov constant, $C_{K}=C_{K A}\left(1+C_{s}\right)^{1 / 3}$ is due to slow mode being passively advected and not contributing to nonlinearity.

We also from these simulations that the residual energy, $E_{B}-E_{v}$ have the same spectral slope as the total energy, i.e., there is a constant fraction of residual energy in the inertial range. The results in Fig. 16 show that residual energy scaling is the same as for total energy so that residual energy is a constant fraction of the total energy. Our best estimate for this fraction is $\sigma_{r}=0.15 \pm 0.03$. More commonly used in the solar wind community, Alfvén ratio $r_{A}=E_{v} / E_{B}=\left(1-\sigma_{r}\right) /\left(1+\sigma_{r}\right) \approx 0.74$. Residual energy and its scale-dependence has been discussed in the past and has recently been associated with the so-called called alignment measures in simulations (Beresnyak and Lazarian 2009a) and in the solar wind measurements (Wicks et al. 2013; Chen et al. 2013). Explaining previously reported -2 scaling (Müller and Grappin 2005) for the residual energy is challenging from the theoretical standpoint. Assuming particular 


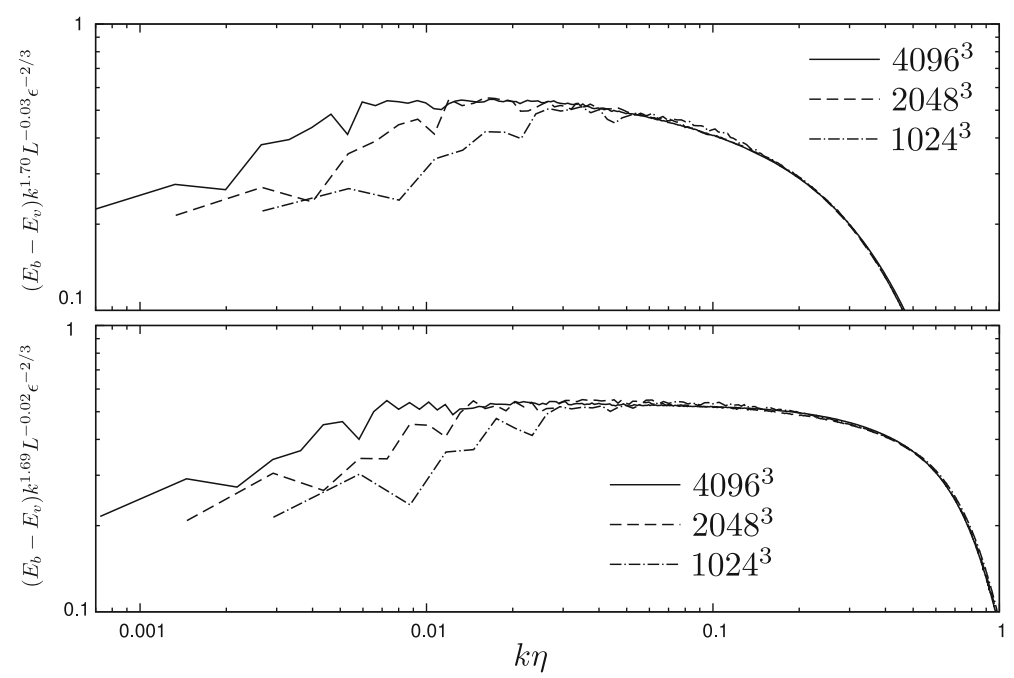

Fig. 16 Residual energy convergence. Best convergence is $k^{-1.70}$ scaling for M1-3 and $k^{-1.69}$ scaling for M1-3H. Image reproduced with permission from Beresnyak (2014); copyright by AAS

residual energy on the outer scale, and the -2 scaling, its value in the inertial range will depend on the scale separation. This would mean a nonlocal character of residual energy. Our simulations, showing that the residual energy is just a fraction of the total energy in the inertial range, resolve this conceptual difficulty and make theories suggesting different scalings for magnetic and kinetic energies obsolete.

The solar wind spectra often feature different kinetic and magnetic scalings, see Fig. 17. The amount of residual energy changes from measurement to measurement and is different for the fast and the slow solar wind (Wicks et al. 2013; Chen et al. 2013). These deviations are not observed in numerics and will be the subject of future study. We optimistically believe that RMHD is valid for large-scale solar wind fluctuations. However, the disagreement between simulations and the measurements could also be due to the solar wind being inhomogeneous, expanding and accelerating (Tenerani and Velli 2017), anisotropic with respect to the sunward direction (Grappin and Velli 1996), and having the large number of discontinuities (Borovsky 2010).

\subsection{Numerics: parallel spectrum}

We plotted the parallel spectrum $E\left(k_{\|}\right)$vs dimensionless wavenumber $k v_{A} \tau_{\eta}$, compensated by $k^{2} \varepsilon^{-1} v_{A}$ to see how the scaling is consistent with (61). This measurement is presented in Fig. 18. For the RMHD case the spectra collapsed, meaning the overall scaling of $k^{-2}$.

Reduced MHD can be performed with different the mean field strength, which in practice requires a particular choice of $\varepsilon$ to generate strong turbulence from the outer scale. The Alfvén symmetry of numerical RMHD formulation ensures that $E\left(k_{\|}\right)$scale precisely linearly $\varepsilon$. However, statistically isotropic MHD simulations with $B_{0}=0$ MHD1-2 do not have this symmetry, and the inertial range scaling (61) cannot be 
Fig. 17 Power spectra of magnetic field, velocity and residual energy measured in the solar wind. Alfvén ratio was strongly fluctuating, and the average was around 0.71 . Image adapted from Chen et al. (2013); copyright by AAS
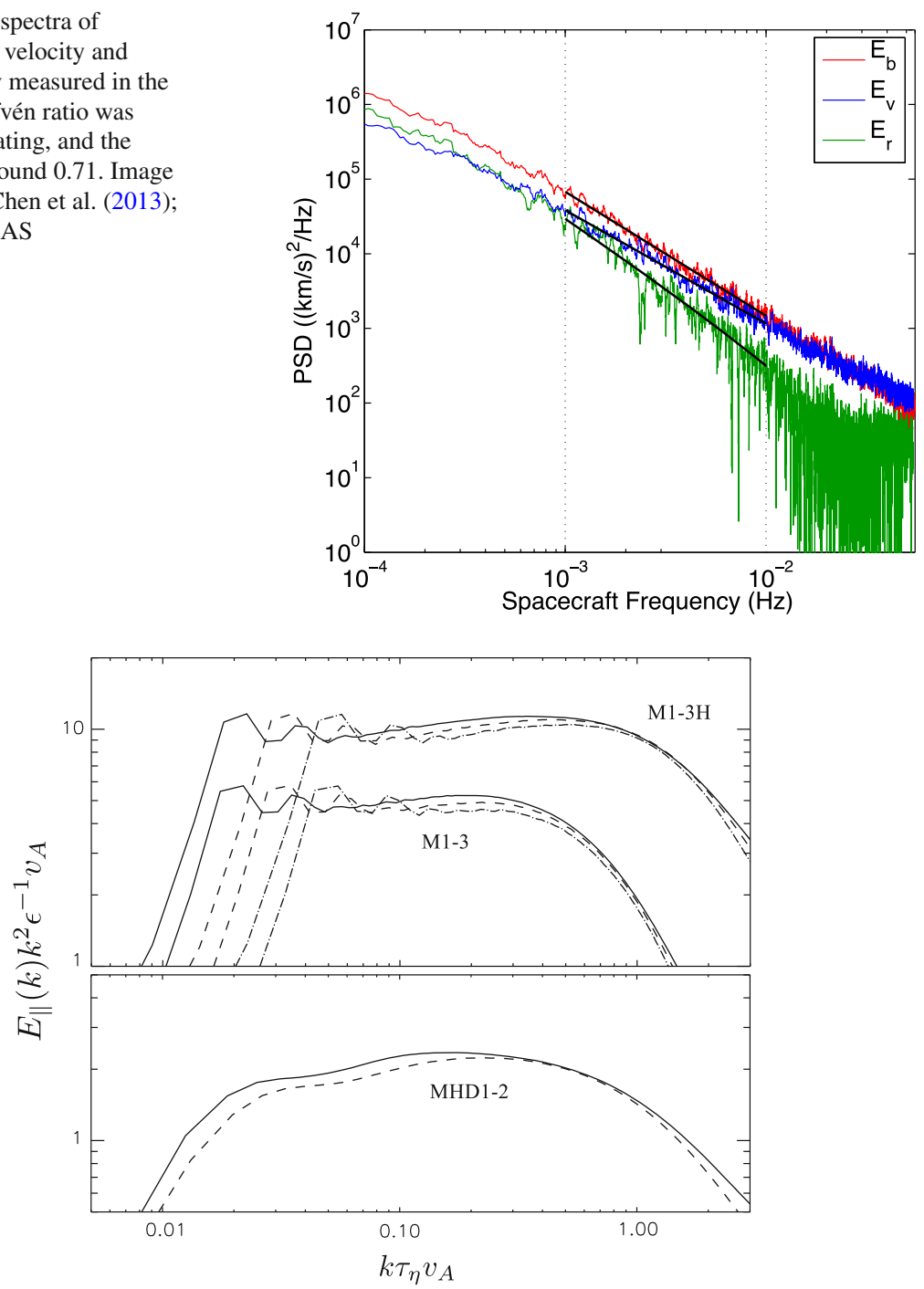

Fig. 18 Energy spectrum along the magnetic field line $E\left(k_{\|}\right)$compensated by the theoretical scaling $\varepsilon k_{\|}^{-2}$ (61). Upper plot: Solid, dashed and dash-dotted are spectra from $4096^{3}, 2048^{3}$ and $1024^{3}$ RMHD simulations. The M1-3H has been multiplied by a factor of two to separate the curves. Lower plot: dashed and solid are MHD1 and MHD2. Image reproduced with permission from Beresnyak (2015); copyright by AAS

rigorously argued based on units. Our test of Eq. (61) is the test not only of the Lagrangian spectrum idea but also the Kraichnan hypothesis of dominant local $v_{A}$. We substituted the RMS field instead of $v_{A}$ in Eq. (61). Figure 18 demonstrates that there is convergence to $\varepsilon k^{-2}$ in this zero mean field case as well.

Another spectral measurement is along the direction of the global mean field in M1-3, M1-3H. We expect these scalings to be the same as the perpendicular scalings, 


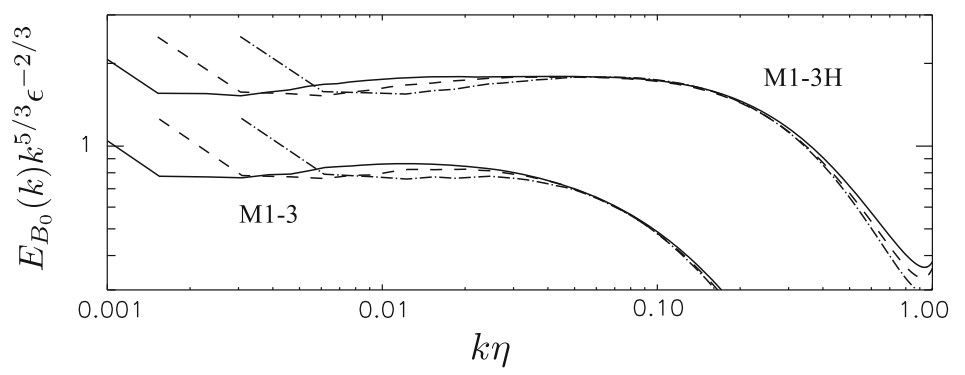

Fig. 19 The spectra along the global mean field in M1-3, M1-3H. The M1-3H spectra have been shifted by a factor of two. The energy spectrum scales as $k^{-5 / 3}$, i.e., in the same way as the perpendicular scaling. Image reproduced with permission from Beresnyak (2015); copyright by AAS

i.e., Kolmogorov because while Alfvén waves propagate along the local field direction which deviates by an angle of $\delta B_{L} / B_{0}$ from $\mathbf{B}_{\mathbf{0}}$, the angular anisotropy in this frame is $\delta B_{l} / B_{0}$, with inertial range values of $\delta B_{l}$ much smaller than the outer scale value of $\delta B_{L}$. It follows that the anisotropy will be washed out. Figure 19 presents a measurement of the spectrum along the global mean field direction, which is consistent with $-5 / 3$.

Worth noting that the application of the critical balance fails in the imbalanced turbulence (more on this in Sect. 8). A more rigorous Lagrangian argument does not have this problem. We can imagine that the energy cascade is manifested both in space and time domains, with the parallel direction is equivalent to the time domain, the anisotropy relation $k_{\|} \sim k_{\perp}^{2 / 3}$ being the correspondence between space domain (Eulerian) and frequency domain (Lagrangian) spectra. Observational data from the solar wind points to the $k^{-2}$ parallel spectrum, e.g., Horbury et al. (2008). Numerical studies overwhelmingly support $k^{-2}$, as long as the measurements was along the local field direction see, e.g., Cho and Vishniac (2000), Maron and Goldreich (2001), Beresnyak and Lazarian (2009a,b), Beresnyak (2012a), while the measurements in the global frame usually demonstrate scale-independent anisotropy, see e.g., Grappin and Müller (2010).

\subsection{Numerics: anisotropy}

We alluded above that the anisotropy should be universal in the inertial range due to the relation between Lagrangian and Eulerian spectra. We expect the relation between parallel and perpendicular scales to follow Eq. (60). Both Alfvénic and slow modes are expected to have the same anisotropy. Similar relation is expected to hold between parallel and perpendicular wavenumber:

$$
k_{\|}=(2 \pi)^{1 / 3} C_{A}^{-1} v_{A}^{-1} k_{\perp}^{2 / 3} \varepsilon^{1 / 3},
$$

In Fig. 20 we plotted wavevector anisotropy. We determine anisotropy relation by solving the equation for $k_{\|}$, given a range of $k_{\perp}$ : 

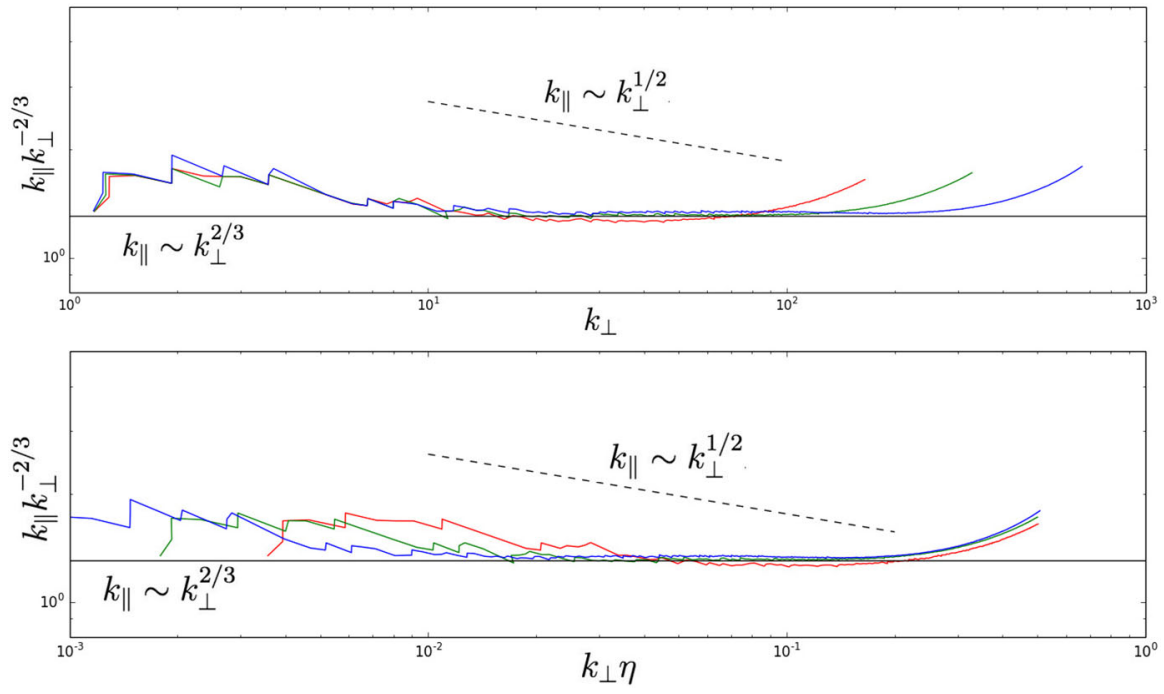

Fig. 20 The relation between parallel and perpendicular wavenumber in Alfvénic turbulence simulations M1-3H. The $k_{\|}$is compensated by $k_{\perp}^{2 / 3}$, which is expected from theory and represented by the solid line. The dashed line corresponds to the theory with $3 / 2$ spectral scaling. Top: $\mathrm{x}$-axis is a dimensional wavenumber, bottom: $\mathrm{x}$-axis is a dimensionless $k_{\perp} \eta$, so this plot corresponds to scaling study for anisotropy

$$
E_{\|}\left(k_{\|}\right)=E\left(k_{\perp}\right) .
$$

A similar procedure is done with parallel and perpendicular second order structure functions to obtain the relation between $\Lambda$ and $\lambda$ of Fig. 21. We also did a convergence study in the same spirit that was done for spectra in previous sections, which are on the bottom parts of each two figures above. We see that the theoretical scalings are followed fairly well. To summarize the above three sections, we showed that the spectrum and anisotropy of Alfvénic turbulence follows the two relations below:

In this section, we argued that the properties of Alfvén and slow components of incompressible MHD turbulence in the inertial range would be determined only by the Alfvén speed $v_{A}$, dissipation rate $\varepsilon$ and the scale of interest $\lambda$. The energy spectrum and anisotropy of Alfvén mode will be expressed as

$$
\begin{aligned}
E(k) & =C_{K} \varepsilon^{2 / 3} k^{-5 / 3}, \\
\Lambda / \lambda & =C_{A} v_{A}(\lambda \varepsilon)^{-1 / 3} .
\end{aligned}
$$

Also, we found numerically that the ratio of kinetic to magnetic energies in the inertial range is constant, $r_{A}=E_{v} / E_{B} \approx 0.74$.

\subsection{Dynamic alignment models}

MHD has more degrees of freedom than hydro, which results, in first-order measures, in two independent dimensionless quantities (four degrees of freedom of $w_{\perp}^{ \pm}$minus 

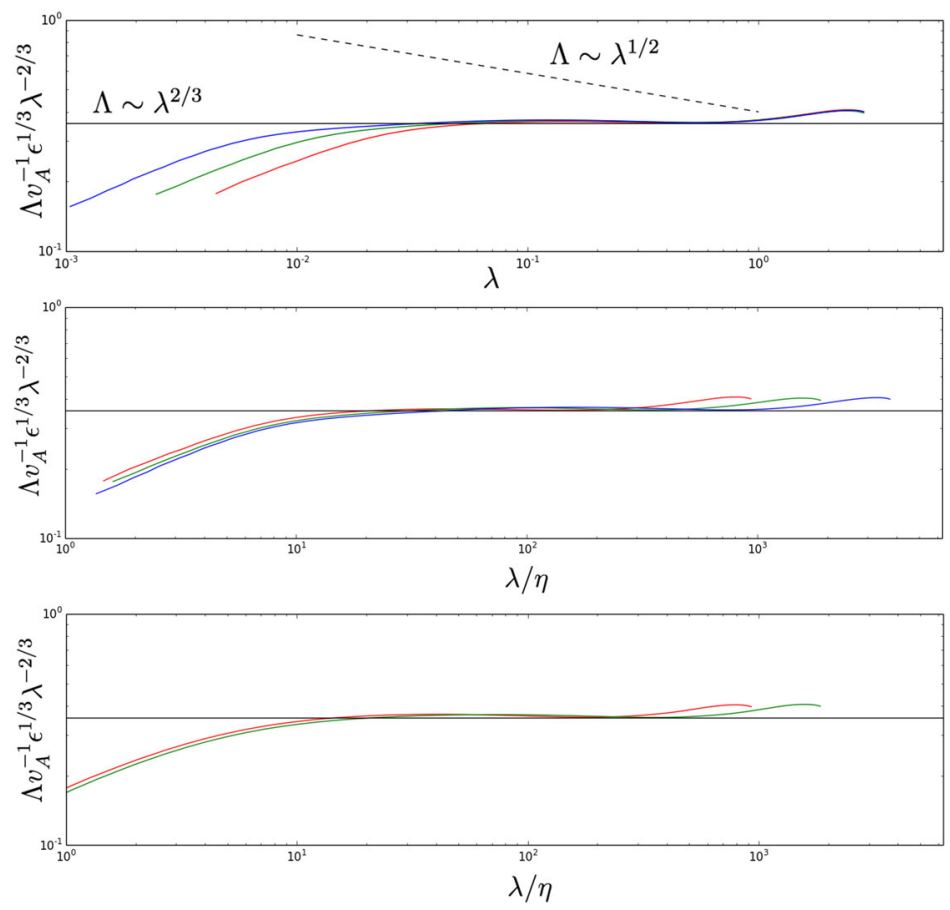

Fig. 21 Anisotropy in real space, the relation between perpendicular scale $\lambda$ and parallel scale $\Lambda$, compensated in this plot by the prefactor $v_{A} \lambda^{-2 / 3} \varepsilon^{1 / 3}$, making it dimensionless. On the top plot we use $\lambda$ on $\mathrm{x}$-axis and plot results from $\mathrm{M} 1-3 \mathrm{H}$. On the middle we use dimensionless $\lambda / \eta$ on $\mathrm{x}$-axis and again plot results from $\mathrm{M} 1-3 \mathrm{H}$. On the bottom we use results from M1-3

rotational freedom minus normalization). One example of this is the fraction of residual energy, introduced earlier. These dimensionless quantities may, in principle, have a non-trivial scaling in the inertial range.

After spectral scaling of $k^{-3 / 2}$ has been found in Müller and Grappin (2005), a number of models have been proposed suggesting that strong turbulence phenomenology have to be modified along the lines of Sect. 4.2 to become consistent with this scaling. Among these models are Boldyrev (2005) and Gogoberidze (2007). A sizable confusion ensued, however to which alignment measure represent the scale-dependent weakening of interaction more accurately. The original Boldyrev (2005) idea was analyzed in Beresnyak and Lazarian (2006) and no significant alignment was found for the averaged angle between $\mathbf{w}^{+}$and $\mathbf{w}^{-}, \mathrm{AA}=\left\langle\left|\delta \mathbf{w}_{\lambda}^{+} \times \delta \mathbf{w}_{\lambda}^{-}\right| /\left|\delta \mathbf{w}_{\lambda}^{+}\right|\left|\delta \mathbf{w}_{\lambda}^{-}\right|\right\rangle$, but when this angle was weighted with the amplitude PI $=\left\langle\left|\delta \mathbf{w}_{\lambda}^{+} \times \delta \mathbf{w}_{\lambda}^{-}\right|\right\rangle /\left\langle\left|\delta \mathbf{w}_{\lambda}^{+}\right|\left|\delta \mathbf{w}_{\lambda}^{-}\right|\right\rangle$, some scale-dependent alignment was found. Later, Boldyrev (2006) proposed the alignment between $\mathbf{v}$ and $\mathbf{b}$ and subsequently Mason et al. (2006) suggested a particular amplitude-weighted measure, DA $=\left\langle\left|\delta \mathbf{v}_{\lambda} \times \delta \mathbf{b}_{\lambda}\right|\right\rangle /\left\langle\left|\delta \mathbf{v}_{\lambda}\right|\left|\delta \mathbf{b}_{\lambda}\right|\right\rangle$, that was claimed to depend of perpendicular scale as $\lambda^{1 / 4}$. In a sense, DA is very similar to PI but uses $\mathbf{B}$ and $\mathbf{v}$ instead of $\mathbf{w}^{ \pm}$. The measure for local imbalance was introduced in Beresnyak and Lazarian (2009a) as IM $=\left\langle\left|\delta\left(w_{\lambda}^{+}\right)^{2}-\delta\left(w_{\lambda}^{-}\right)^{2}\right|\right\rangle /\left\langle\delta\left(w_{\lambda}^{+}\right)^{2}+\delta\left(w_{\lambda}^{-}\right)^{2}\right\rangle$. While reevaluating the logic in Boldyrev (2006) it becomes clear that the choice of DA as a 


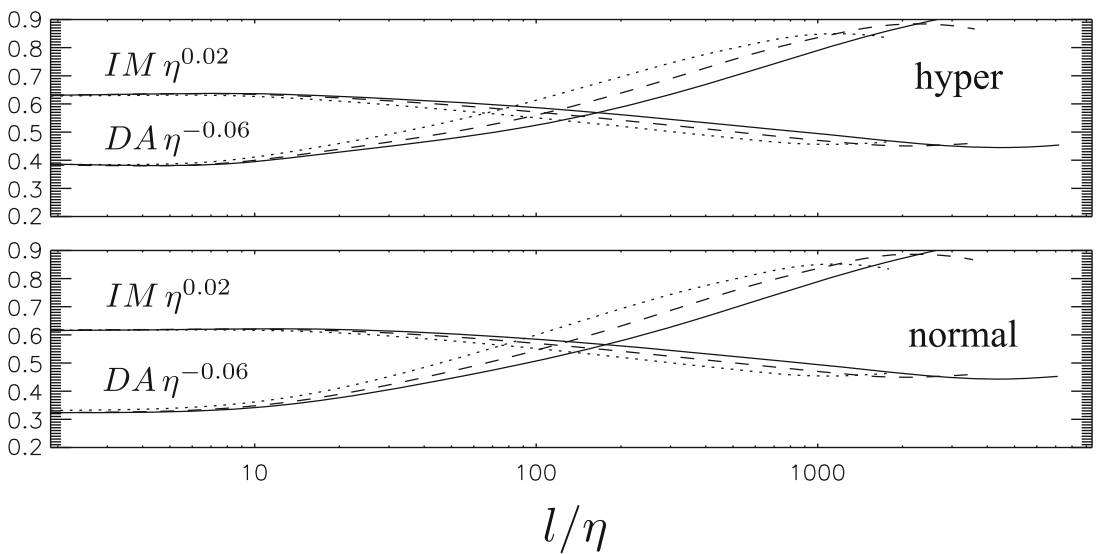

Fig. 22 Scaling study of alignment measures DA $=\langle|\delta \mathbf{v} \times \delta \mathbf{b}|\rangle /\langle|\delta v \delta b|\rangle$ and $\mathrm{IM}=\langle| \delta\left(w^{+}\right)^{2}-$ $\delta\left(w^{-}\right)^{2}|\rangle /\left\langle\delta\left(w^{+}\right)^{2}+\delta\left(w^{-}\right)^{2}\right\rangle$ from M1-3H (top) and M1-3 (bottom). The alignment slopes converge to relatively small values, e.g., 0.06 for DA which is smaller than $1 / 4$, predicted in alignment theories. Image reproduced with permission from Beresnyak (2015); copyright by AAS, see also Beresnyak and Lazarian (2009a), Beresnyak (2011, 2012a)

measure exclusively responsible for the interaction weakening is arbitrary, at the same time the argument that DA scales as $l^{1 / 4}$ due to field line wandering, is invalid keeping in mind the Alfvén symmetry of RMHD equations. The argument in Boldyrev (2006) suggests that DA will tend to increase, but will be bounded by field wandering, i.e., the alignment on each scale will be created independently of other scales and will be proportional to the relative perturbation amplitude $\delta B / B_{0}$. However, this violates Alfvén symmetry of RMHD equations (see Sect. 7.2), which requires that $B_{0}$ can be factored out of the dynamics and appear only in combination with $k_{\|}$. The most apparent contradiction we find while following Boldyrev (2006) is that a perfectly aligned state, e.g., with $\delta \mathbf{w}^{-}=0$ is a precise solution of MHD equations and it is not destroyed by its field wandering. Empirically, we know that alignment measures showed very little or no dependence on $\delta B_{L} / B_{0}$ (see, e.g., Beresnyak and Lazarian 2009a).

Figure 22 studies scale-dependency of DA and IM by the method of scaling study. The asymptotic scale-dependency slope for DA for our data is found around 0.06, which is way below its value of $1 / 4$ suggested in Boldyrev (2006). From this figure it is evident that the scale-dependency of DA seems is tied to the outer scale, i.e., it is non-universal.

\section{Imbalanced MHD turbulence}

While hydrodynamic turbulence has only one energy cascade, the incompressible MHD turbulence has two, due to the exact conservation of the Elsässer (oppositely going wave packets') "energies". The situation of zero total cross-helicity, which we considered in previous sections has been called "balanced" turbulence as the amount of 
Fig. 23 Spectra of imbalanced turbulence measured in the solar wind. Trace of the Fourier and wavelet power spectra of $\left(w^{+}\right)^{2}$ (black line and red symbols) and $\left(w^{-}\right)^{2}$ (gray line and blue symbols) parallel and perpendicular to the local magnetic field. The bottom panel shows the ratio $\left(w^{+} / w^{-}\right)^{2}$. Image reproduced with permission from Wicks et al. (2011); copyright by APS

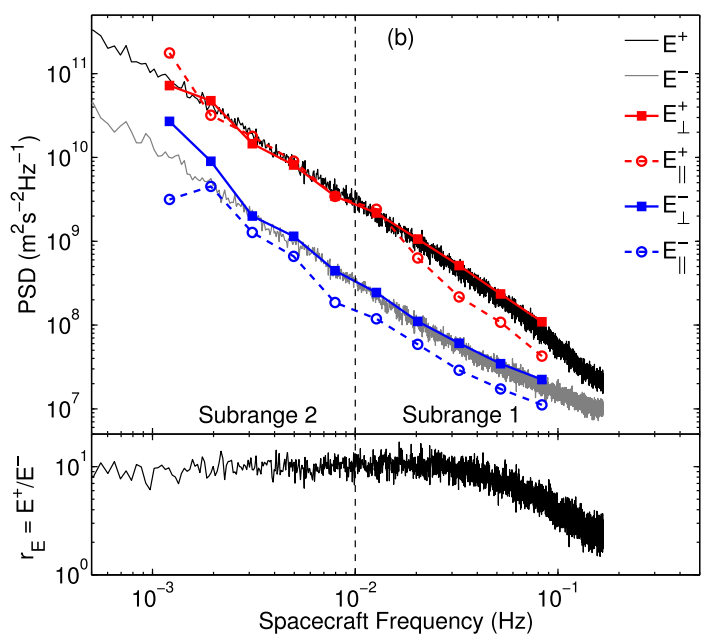

oppositely moving wavepackets balance each other, the alternative being "imbalanced" turbulence. Imbalanced turbulence, however, is very common, it is enough to have a mean magnetic field (like in the ISM) and a localized source of perturbations. Another example is the solar wind, where the dominant perturbation component propagate away from the Sun, see Fig. 23. Likewise, we expect similar phenomena in active galactic nuclei (AGN), where the jet has a mean magnetic field component and the perturbations will propagate primarily away from the central engine.

\subsection{Theoretical considerations}

A conceptual difficulty in the imbalanced case arises from the application of the critical balance idea. If $\delta w_{l}^{+}$critical balance depends on $\delta w_{l}^{-}$amplitude, their parallel scales and frequencies are mismatched, and the cascade cannot proceed in a normal manner. Below we describe three models that tried to deal with this issue, references are Lithwick et al. (2007) (LGS07), Beresnyak and Lazarian (2008) (BL08), and Perez and Boldyrev (2009) (PB09). In short, only BL08 model is consistent with all numerical evidence, taking into account cascading rates, spectra and anisotropy. Below we shortly describe these theories.

PB09 employs dynamic alignment which depends on the scale as $l^{1 / 4}$, this alignment, however, is acting differently on $w^{+}$and $w^{-}$so that it effectively results in the same nonlinear timescales for both components. It could be rephrased that PB09 predicts turbulent viscosity on each scale which is equal for both components. this results in an expression for the ratios of energies

$$
\left(w^{+}\right)^{2} /\left(w^{-}\right)^{2}=\varepsilon^{+} / \varepsilon^{-} \quad \text { for (PB09). }
$$

It is not clear how this is consistent with the limit of large imbalances, where the weak component will not be able to produce any sizable interaction. 
Fig. 24 Upper: a $\mathbf{w}^{+}$ wavepacket, produced by cascading by $\mathbf{w}^{-}$wavepacket is aligned with respect to $\mathbf{w}^{-}$ wavepacket, but misaligned with respect to the local mean field on scale $\lambda_{1}$, by the angle $\theta$. Lower: the longitudinal scale $\Lambda$ of the wavepackets, as a function of their transverse scale, $\lambda ; \Lambda^{+}$, $\Lambda^{-}, \lambda_{1}, \lambda_{2}$ are the notations used in this review. Image reproduced with permission from Beresnyak and Lazarian (2008); copyright by AAS

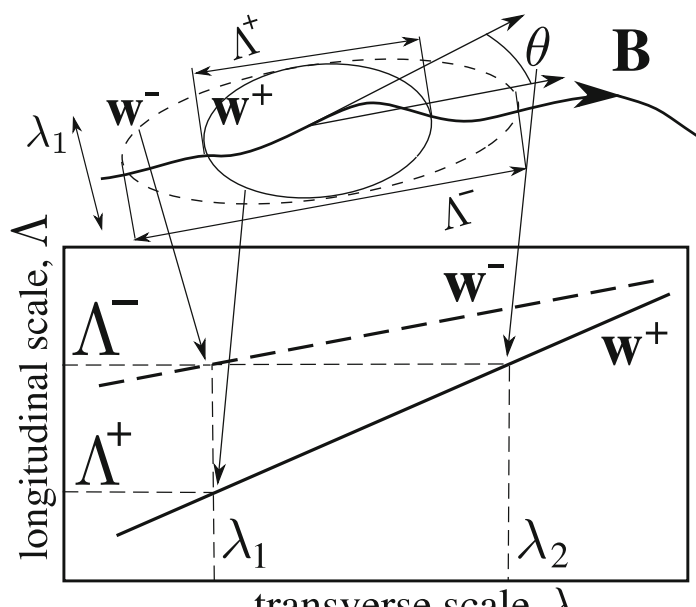

transverse scale, $\lambda$

In LGS07 the authors proposed that the parallel scale for both components is determined by the shear rate of the stronger component, despite the cascading timescale is different for both components. The energy cascade is still a strong cascade:

$$
\varepsilon^{\mp}=\frac{\left(w^{\mp}(\lambda)\right)^{2} w^{ \pm}(\lambda)}{\lambda} \quad \text { for (LGS07). }
$$

This results in the prediction

$$
w^{+} / w^{-}=\varepsilon^{+} / \varepsilon^{-} \quad \text { for (LGS07). }
$$

Both PB09 and LGS07 predict the same anisotropy for both components.

In BL08 the authors proposed a new formulation of critical balance for the stronger component. This model is described in greater detail below. BL08 relaxes the assumption of local cascading for the strong component $w^{+}$, while saying the $w^{-}$classic critical balance and local cascading. In the BL08 picture the waves have different anisotropies (see Fig. 24) and the $w^{+}$wave have smaller anisotropy than $w^{-}$, which is opposite to what a naive application of critical balance would predict. The anisotropies of the waves are determined by

$$
\begin{aligned}
& w^{+}\left(\lambda_{1}\right) \Lambda^{-}\left(\lambda_{1}\right)=v_{A} \lambda_{1}, \\
& w^{+}\left(\lambda_{2}\right) \Lambda^{+}\left(\lambda^{*}\right)=v_{A} \lambda_{1},
\end{aligned}
$$

where $\lambda^{*}=\sqrt{\lambda_{1} \lambda_{2}}$, and the energy cascading is determined by weak cascading of the dominant wave and strong cascading of the subdominant wave:

$$
\begin{aligned}
& \varepsilon^{+}=\frac{\left(w^{+}\left(\lambda_{2}\right)\right)^{2} w^{-}\left(\lambda_{1}\right)}{\lambda_{1}} \cdot \frac{w^{-}\left(\lambda_{1}\right) \Lambda^{-}\left(\lambda_{1}\right)}{v_{A} \lambda_{1}} \cdot f\left(\lambda_{1} / \lambda_{2}\right), \\
& \varepsilon^{-}=\frac{\left(w^{-}\left(\lambda_{1}\right)\right)^{2} w^{+}\left(\lambda_{1}\right)}{\lambda_{1}}
\end{aligned}
$$


Table 2 Three-dimensional RMHD imbalanced simulations. Table reproduced with permission from Beresnyak and Lazarian (2015), copyright by Springer

\begin{tabular}{llllll}
\hline Run & Resolution & $f$ & Dissipation & $\varepsilon^{+} / \varepsilon^{-}$ & $\left(w^{+}\right)^{2} /\left(w^{-}\right)^{2}$ \\
\hline I1 & $512 \cdot 1024^{2}$ & $w^{ \pm}$ & $-1.9 \cdot 10^{-4} k^{2}$ & 1.187 & $1.35 \pm 0.04$ \\
I2 & $768^{3}$ & $w^{ \pm}$ & $-6.8 \cdot 10^{-14} k^{6}$ & 1.187 & $1.42 \pm 0.04$ \\
I3 & $512 \cdot 1024^{2}$ & $w^{ \pm}$ & $-1.9 \cdot 10^{-4} k^{2}$ & 1.412 & $1.88 \pm 0.04$ \\
I4 & $768^{3}$ & $w^{ \pm}$ & $-6.8 \cdot 10^{-14} k^{6}$ & 1.412 & $1.98 \pm 0.03$ \\
I5 & $1024 \cdot 1536^{2}$ & $w^{ \pm}$ & $-1.5 \cdot 10^{-15} k^{6}$ & 2 & $5.57 \pm 0.08$ \\
I6 & $1024 \cdot 1536^{2}$ & $w^{ \pm}$ & $-1.5 \cdot 10^{-15} k^{6}$ & 4.5 & $45.2 \pm 1.5$ \\
\hline
\end{tabular}

The BL08 model, unlike LGS07, does not produce self-similar (power-law) solutions when turbulence is driven with the same anisotropy for $w^{+}$and $w^{-}$on the outer scale. BL08, however, claims that, on sufficiently small scales, the initial non-powerlaw solution will transit into asymptotic power law solution that has $\Lambda_{0}^{-} / \Lambda_{0}^{+}=\varepsilon^{+} / \varepsilon^{-}$ and $\lambda_{2} / \lambda_{1}=\left(\varepsilon^{+} / \varepsilon^{-}\right)^{3 / 2}$. The larger imbalance will require larger transition to this asymptotic regime.

\subsection{Numerics}

Table 2 summarizes RMHD simulations with imbalanced driving. In these simulations, we kept the energy injection rate constant. All experiments were evolved into the stationary state. The imbalanced runs have to be were evolved for a longer time to achieve stationary state due to longer cascading timescales for the stronger component.

Compared to spectral slopes, dissipation rates are robust quantities that require much smaller dynamical range and resolution to converge. Figure 25 shows energy imbalance $\left(w^{+}\right)^{2} /\left(w^{-}\right)^{2}$ versus dissipation rate imbalance $\varepsilon^{+} / \varepsilon^{-}$for simulations I2, I4, I5 and I6. We also use two data points from earlier simulations with large imbalances, A7 and A5 from Beresnyak and Lazarian (2009b). I1 and I3 are simulations with the normal viscosity similar to I2 and I4. They show slightly less energy imbalances than I2 and I4. We see that most data points are above the prediction of LGS07, which is consistent with BL08. In other words, numerics strongly suggest that

$$
\frac{\left(w^{+}\right)^{2}}{\left(w^{-}\right)^{2}} \geq\left(\frac{\varepsilon^{+}}{\varepsilon^{-}}\right)^{2} \text {. }
$$

Although there is a tentative correspondence between LGS07 and the data for small degrees of imbalance, the deviations for large imbalances are significant. As to PB09 prediction, it is inconsistent with data for all degrees of imbalance including those with small imbalances.

The approximate equality in Eq. (74) for very small imbalances, however, is an excellent test of the expression in Eq. (58) that we assumed in the balanced case. So in some sense, empirical study of the imbalanced case validated the theory of the balanced case as well. 


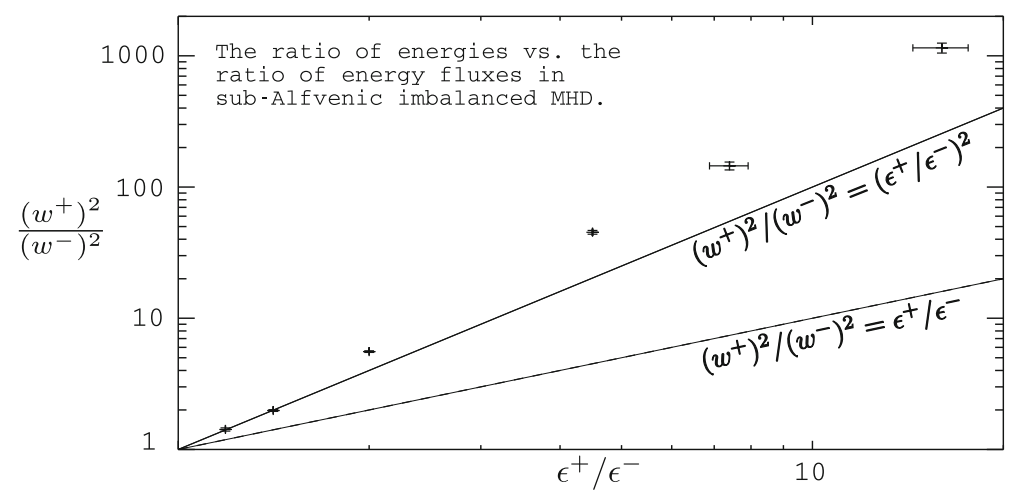

Fig. 25 Elsässer energy ratio plotted versus dissipation rate ratio in simulations I2, I4, I5, I6 (Table 2) and A7 and A5 from Beresnyak and Lazarian (2009b). The solid line is the LGS07 prediction, and the dashed line is a PB09 prediction, also a prediction for purely viscous/non-turbulent dissipation of eddies. Image reproduced with permission from Beresnyak and Lazarian (2015), copyright by Springer
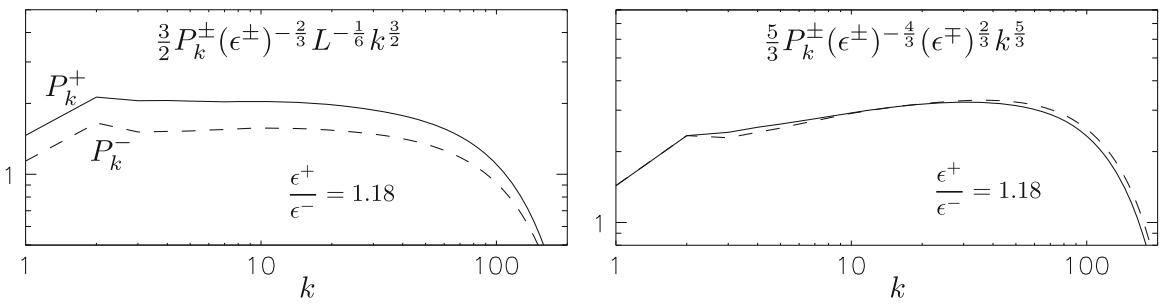

Fig. 26 Energy spectra for $w^{+}$(solid) and $w^{-}$(dashed) from simulation I2, compensated by factors that correspond to PB09 (left) and LGS07 (right). Spectra collapse on the right, but not on the left. Image reproduced with permission from Beresnyak and Lazarian (2015), copyright by Springer

Figure 26 shows spectra from low-imbalance simulation I2, compensated by the predictions of PB09 and LGS07. We see that the collapse of two curves for $w^{+}$and $w^{-}$is much better for the LGS07 model.

Figure 27 shows spectra from all I1-6 simulations, compensated by the prediction of LGS07. For lower imbalances, the collapse is reasonably good and become progressively worse for larger imbalances. This deviation, however, does not entirely follow the prediction of the asymptotic power-law solutions from BL08, which will predict that the solid curve will go above $C_{K A}$ and the dashed curve-below it. This is possibly explained by the fact that asymptotic power-law solutions were not reached in these limited resolution experiments, this is also observed for anisotropies.

We measured parallel and perpendicular structure functions in simulations I1-I6, in order to quantify the anisotropies of eddies.

Figure 28 shows anisotropies for I1-6 simulations.

All simulations were driven by the same anisotropies on the outer scale, which is unfavorable for obtaining the asymptotic power law solutions of BL08. It is, however, favorable to the LGS07 model, which predicts the same $w^{+}$and $w^{-}$anisotropies for all scales. Therefore, these simulations are a sensitive test between LGS07 and BL08 models, both of which are roughly consistent in terms of energy ratios and spectra for small 


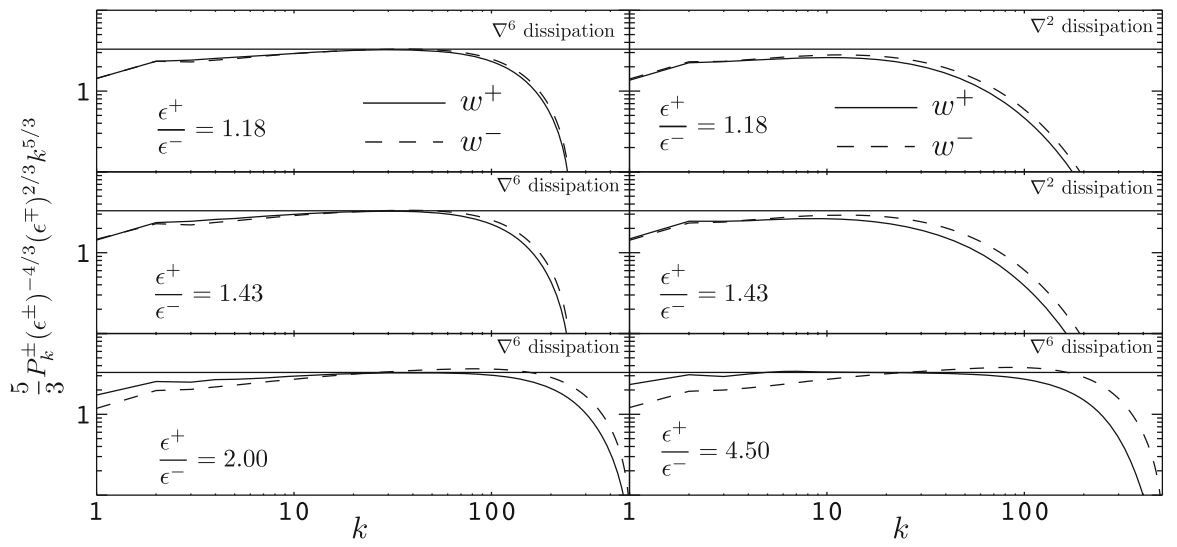

Fig. 27 Energy spectra for $w^{+}$(solid) and $w^{-}$(dashed) for simulations I1-I6, compensated by factors that correspond to LGS07. The thin solid line corresponds to Kolmogorov constant for Alfvénic turbulence $C_{K A}=3.27$. Image reproduced with permission from Beresnyak and Lazarian (2015), copyright by Springer

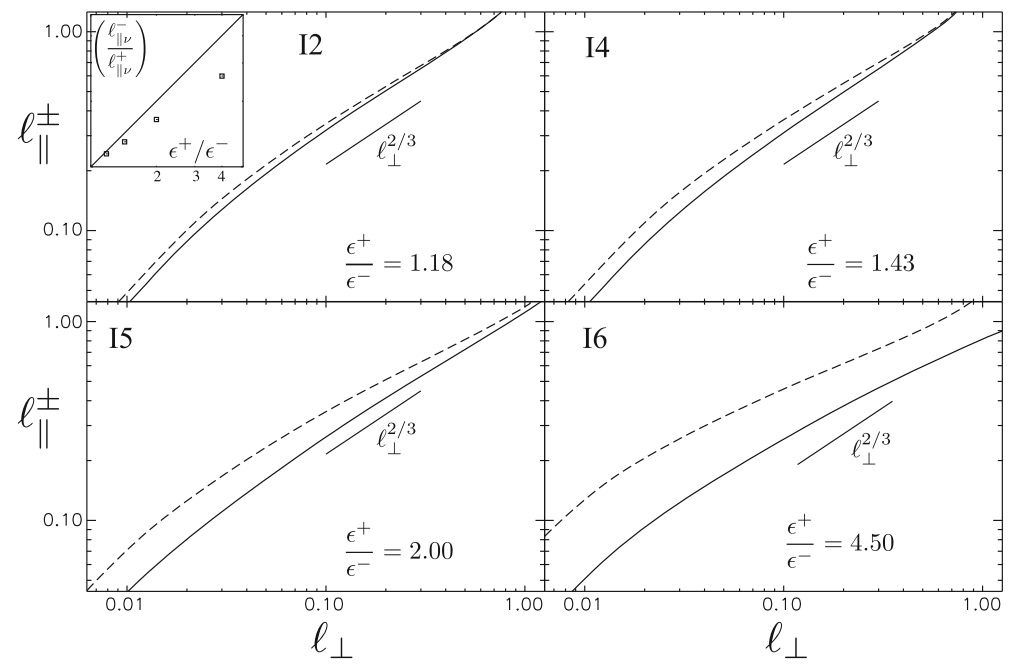

Fig. 28 Anisotropies for $w^{+}$(solid) and $w^{-}$(dashed), simulations I1-I6. The small upper inset shows the ratio of anisotropies on the smallest scales vs. the prediction of BL08, $\varepsilon^{+} / \varepsilon^{-}$. Image reproduced with permission from Beresnyak and Lazarian (2015), copyright by Springer

imbalances. If the LGS07 model is correct, we would observe the same anisotropy on all scales, but this is not what is observed in Fig. 28, where anisotropies start to diverge on smaller scales. The ratio of anisotropies is roughly consistent with the BL08 prediction of $\varepsilon^{+} / \varepsilon^{-}$for small imbalances and somewhat smaller for larger imbalances.

\section{MHD dynamo}

One of the main questions of MHD dynamics is how conductive fluid generates its magnetic field, a process known broadly as "dynamo". Turbulent dynamo is known 
as "large-scale/mean-field dynamo" and "small-scale/fluctuation dynamo", in the first case magnetic fields are amplified on scales larger than the outer scale of turbulence in the seconds on smaller scales. An example of the flow generating no dynamo is an axisymmetric situation (Cowling 1933), the natural turbulence, however, possesses no exact symmetries and is expected to amplify magnetic field by stretching, due to the particle separation in a turbulent flow. For the large-scale dynamo, a "twist-stretchfold" mechanism was introduced in Vainshtein and Zeldovich (1972).

If the turbulent flow possess statistical isotropy, it can not generate a large-scale field, i.e., the field with scales larger than the outer scale of turbulence. To generate the observed large-scale fields, such as fields in the disk galaxies, large-scale asymmetries of the system must break the statistical symmetry of turbulence. Further analysis of the induction equation shows that the rotation (described by the pseudovector of angular velocity) is insufficient to provide large-scale dynamo, stratification or shear should provide a real vector, so that the pseudoscalar alpha-effect result in a large-scale turbulent EMF (Vishniac and Cho 2001; Käpylä et al. 2009).

One approach to large-scale dynamo is mean field theory, see, e.g. Krause and Raedler (1980), where the magnetic and velocity fields are decomposed into the mean and fluctuating part. The equations for the mean field are closed using statistical or volume averaging over the fluctuating part. The traditional theories of mean field dynamo, however, often fail due to issues related to magnetic helicity (Vishniac and Cho 2001; Vishniac et al. 2003).

The studied of the large-scale dynamos is big and complex science due to the variety of ways, large-scale symmetries are broken in different astrophysical objects. In this review, we propose the reader follow other reviews focused on large-scale dynamos, e.g., Brandenburg and Subramanian (2005); Charbonneau (2010) and instead concentrate on the small-scale or fluctuating dynamo as more universal, generic and crucial to understand the overall level of magnetization in astrophysical environments. We also emphasize that proper understanding of turbulent magnetic fields is crucial as it is subsequently slowly ordered and made large-scale by the large-scale dynamo process.

\subsection{Kinematic dynamo}

If the magnetic energy is less than the kinetic energy of turbulent motions, the turbulence may generate the magnetic field, which is referred to as "turbulent dynamo". There are two distinct regimes: 1) the magnetic energy is much less than the kinetic energy of driving eddies at all scales down to the dissipation scale and 2) the kinetic and magnetic energies come to the equipartition at some scale. The first regime is called "kinematic" or "linear" dynamo, referring to the induction equation (35) being linear with respect to the magnetic field. If the magnetic field is so weak that it provides no back-reaction to velocity, the problem reduces to studying the solutions of the induction equation with a prescribed velocity field. This kinematic regime was, historically, the most studied (see the Kazantsev model outlined in Kazantsev 1968; Kraichnan and Nagarajan 1967; Kulsrud and Anderson 1992; Vincenzi 2002). For the Kolmogorov-type turbulence, the fastest magnetic field amplification comes from the fastest turbulent eddies, i.e., the eddies at the dissipation scales. It would follow that 
the growth rate $\gamma=1 / \tau_{\eta}$ (see Eq. (26)). The spectrum of the magnetic field will be $E(k) \sim k^{3 / 2}$ rising sharply to the magnetic dissipation scale. Kazantzev picture was not without drawbacks since it had relied on an artificial delta-correlated velocity field instead of a realistic turbulent velocity field. This resulted in an overestimated $\gamma$ compared to reality. Precise numerical experiments with $\operatorname{Pr}_{m}=\operatorname{Re}_{m} / \mathrm{Re}=1$ have found a prefactor $\gamma \tau_{\eta}=0.0326$ (Haugen et al. 2004; Schekochihin et al. 2004; Beresnyak $2012 \mathrm{~b}$ ) which is much smaller than unity. This small number, as had been suggested in Beresnyak (2012b) is due to turbulence being time-asymmetric.

From kinematic models, it is not clear whether magnetic energy will continue to grow after the end of the kinematic regime, however, keeping in mind $\gamma \sim \operatorname{Re}^{1 / 2}$ and very large astrophysical Re, the kinematic growth is incredibly fast and occupies a tiny fraction of the dynamical time of the system. For example, while the galaxy clusters form on timescales of 15 billion years, the characteristic growth time of kinematic dynamo is less than a million years (Beresnyak and Miniati 2016), so we do not expect kinematic dynamo to operate in present time. The magnetic spectrum of the kinematic dynamo, with its positive spectral index, is incompatible with observations in galaxy clusters (Laing et al. 2008), see Fig. 1. These observations indicate steep spectrum with negative power index at small scales.

Summarizing, the kinematic dynamo is inapplicable in most astrophysical environments, since the observed magnetization corresponds to Alfvén speed which is many orders of magnitude higher than the Kolmogorov velocity $\varepsilon^{1 / 4} v^{1 / 4}$.

\subsection{Nonlinear dynamo}

Before numerical simulations were commonplace, it was proposed that after saturation of kinematic dynamo the magnetic energy will stop growing. If we agree to this proposition and assume that the magnetic energy indeed saturates as soon as the dynamo becomes nonlinear, then the saturation level, in this case, will be of order $\rho v_{\eta}^{2} / 2$, where $v_{\eta}$ is a Kolmogorov velocity scale. This is a factor of $\operatorname{Re}^{-1 / 2}$ smaller than the kinetic energy density and will be completely dynamically unimportant in high-Re astrophysical environments. In fact, observations indicate the opposite-a sizable energy density of the magnetic field in large-scale systems, see Fig. 1

An alternative had been proposed in the early work by Schlüter and Biermann (1950), who suggested that dynamo will not stop and will continue to grow, saturating on each subsequent scale after a dynamical time. Recently small-scale dynamo underwent revival due to the availability of direct numerical simulations. Simulations of the dynamo saturated state produced steep spectra and significant outer-scale fields. The saturated state was only weakly dependent on $\operatorname{Re}$ and $\operatorname{Pr}_{m}$ as long as $\operatorname{Re}$ was large, see, e.g., Haugen et al. (2004).

Apart from the saturated state, the growth stage was suggested to have growth of magnetic energy which is linear in time (Schekochihin and Cowley 2007; Cho et al. 2009; Ryu et al. 2008; Beresnyak et al. 2009; Beresnyak 2012b). In Beresnyak (2012b) the locality of the dynamo, which is necessary for the linear growth picture was argued analytically. Let us imagine that the magnetic and kinetic spectra at a particular moment of time are similar to what is presented in Fig. 29. Magnetic and kinetic spectra cross at 


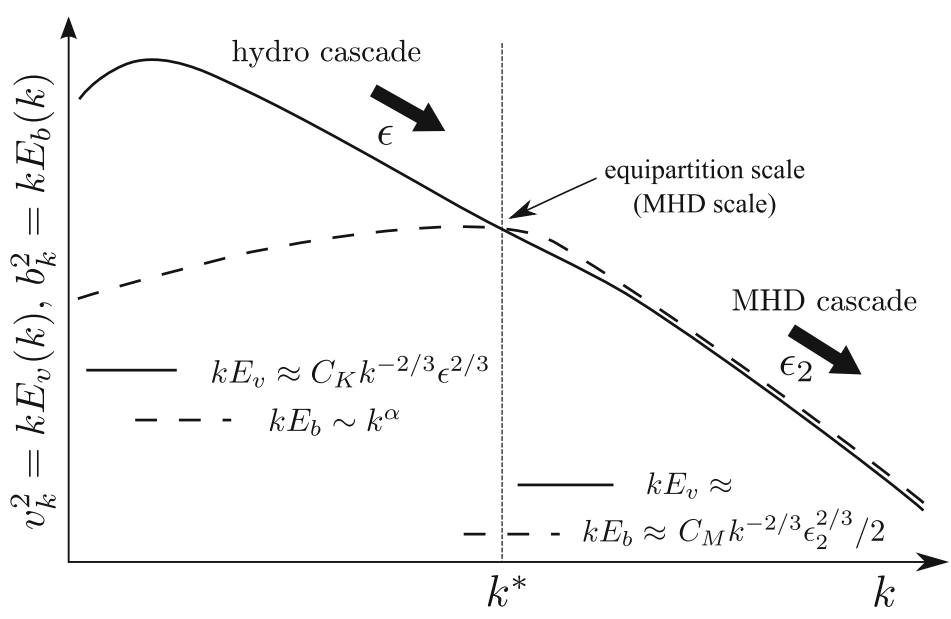

Fig. 29 A cartoon of kinetic and magnetic spectra in small-scale dynamo, at a particular moment of time when equipartition wavenumber is $k^{*}$. Image reproduced with permission from Beresnyak and Lazarian (2019); copyright by De Gruyter

some "equipartition" scale $1 / k^{*}$, below which both spectra are steep, typically $k^{-5 / 3}$ due to the MHD cascade (Sect. 7). A number of arguments suggest this assumption. Firstly, we expect kinematic dynamo (Kulsrud and Anderson 1992) to proceed until the moment when magnetic spectrum intersect the kinetic spectrum at the viscous scales (assuming $\operatorname{Pr}_{m}=1$ ), which will correspond to the beginning of the nonlinear regime. Secondly, this is supported by the wealth of numerics, e.g., Beresnyak and Lazarian (2009a); Cho et al. (2009). Thirdly, this is also somewhat supported by observations of magnetic fields in clusters (Laing et al. 2008).

At large scales magnetic spectrum is shallow, $k^{\alpha}, \alpha>0$, while kinetic spectrum is steep due to the hydrodynamic cascade. Most of the magnetic energy is contained at the scale of $1 / k^{*}$. We designate $C_{K}$ and $C_{M}$ as Kolmogorov constants of hydro and MHD respectively. The hydrodynamic cascade rate is $\varepsilon$ and the MHD cascade rate as $\varepsilon_{2}$. Due to the conservation of energy in the inertial range, magnetic energy will grow at a rate of $\varepsilon-\varepsilon_{2}$. We will designate $C_{E}=\left(\varepsilon-\varepsilon_{2}\right) / \varepsilon$ as an "efficiency of the smallscale dynamo" and will argue that this is a true constant, since a) turbulent dynamics is local in scale in the inertial range; b) ideal MHD or Euler equations do not contain any scale explicitly. Magnetic energy will grow linearly with time if $\varepsilon=$ const:

$$
\frac{1}{8 \pi} \frac{d B^{2}}{d t}=C_{E} \varepsilon
$$

The equipartition scale $L_{B}=1 / k^{*}$ will grow with time as $t^{3 / 2}$ (Beresnyak et al. 2009):

$$
L_{B}=c_{l} v_{A}^{3} / \varepsilon_{\text {turb }},
$$

here we introduced the dimensionless constant $c_{l}$. Alternatively one can say that smallscale dynamo saturates at several dynamical times at scale $1 / k^{*}$ and proceeds to a 
twice larger scale (Schlüter and Biermann 1950; Schekochihin and Cowley 2007). If magnetic energy grows approximately till equipartition (Haugen et al. 2004; Cho et al. 2009), the whole process will take around several outer timescales of the system, or more quantitatively, $\left(C_{K}^{3 / 2} / C_{E}\right)\left(L / v_{L}\right)$.

It was demonstrated analytically in Beresnyak (2012b) that as long as the kinetic spectrum is steep (spectral slope between -1 and -3 ), the magnetic spectrum is steep below the equipartition scale and magnetic spectrum is shallow (slope higher than 0 ) above the equipartition scale, the dynamo is indeed local and the picture described above can indeed be rigorously argued. So, besides the fact that local interactions dominate the kinetic cascade at large scales and the MHD cascade at small scales, we also know that dynamo is governed by local interactions. Assuming Kolmogorov phenomenology, it is also possible to estimate the upper limit on the wavevector interval in which nonlinear dynamo operates, namely the interval $k^{*}\left[C_{E}^{3 / 2} C_{K}^{-9 / 4}, C_{E}^{-3 / 2} C_{M}^{9 / 4}\right]$. Substituting numerically known values $C_{K}=1.6, C_{M}=4.2, C_{E}=0.05$ we get the interval of $k^{*}[0.004,2000]$, which not very restrictive as a practical upper limit for numerical simulations, but undoubtedly essential for astrophysical situations where the inertial range is many orders of magnitude in scale. Given the sufficiently large inertial range, $C_{E}$ a universal dimensionless constant of MHD dynamics, much like Kolmogorov constant and since it relates energy fluxes, not energies, it is also unaffected by intermittency. Interestingly, magnetic energy dynamics at $k \ll k^{*}$ is likely dominated by nonlocal interactions with $k^{*}$ but this part of the spectrum contains negligible magnetic energy and the universality claim is unaffected by this nonlocality.

\subsection{Numerical results}

Numerical simulations of statistically homogeneous isotropic small-scale dynamo in Beresnyak (2012b) were performed by solving MHD equations with stochastic nonhelical driving and explicit dissipation with $P r_{m}=1$. The results in Fig. 30 is the statistical average over three different simulations. Growth is initially exponential and smoothly transition into the linear stage. Note that scatter is initially small, but grows with time, which is consistent with the picture of the magnetic field that grows at the progressively larger scales, and has progressively less independent realizations in a single datacube. The value of the dynamo efficiency that we measure $C_{E}=0.05$ is much smaller than unity. One would expect this quantity of order unity because this is a universal number, determined only by strong interaction on the equipartition scale. If we refer to the ideal incompressible MHD equations, written in terms of Elsässer variables, $\partial_{t} \mathbf{w}^{ \pm}+\hat{S}\left(\mathbf{w}^{\mp} \cdot \nabla\right) \mathbf{w}^{ \pm}=0$, dynamo could be understood as decorrelation of $\mathbf{w}^{ \pm}$which are originally equal to each other in the hydrodynamic cascade. In our case, this decorrelation is happening at the equipartition scale $1 / k^{*}$. Being time-dependent, it propagates upscale, while, ordinarily, energy cascade goes downscale. The small value of $C_{E}$ might be due to this. As opposed to picture with multiple reversals and dissipation due to microscopic diffusivity, typical for the kinematic case, in our picture we appeal to turbulent diffusion which helps to create the large-scale field. Both stretching and diffusion depend on turbulence at the same designated scale $1 / k^{*}$, so, in the asymptotic regime of large $\mathrm{Re}$, one of these processes must dominate. As $C_{E}$ is small, we conclude 


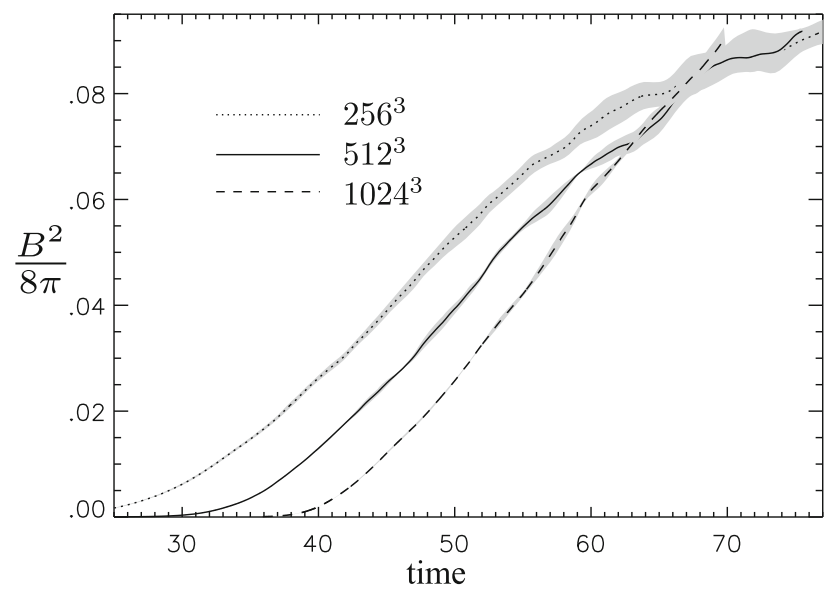

Fig. 30 Magnetic energy growth vs. time in code units, observed in simulations with $\operatorname{Re}_{m}=1000\left(\tau_{\eta}=\right.$ 0.091 in code units), $\operatorname{Re}_{m}=2600\left(\tau_{\eta}=0.057\right)$ and $\operatorname{Re}_{m}=6600\left(\tau_{\eta}=0.036\right)$. We used sample averages which greatly reduced fluctuations and allowed us to measure $C_{E}$ with sufficient precision. Image reproduced with permission from Beresnyak and Lazarian (2015), copyright by Springer

that stretching and diffusion are close to canceling each other. In Beresnyak (2012b), the interplay of stretching and mixing was studied by simulations of kinematic dynamo forward and backward in time. Basically, forward in time stretching is less efficient, while mixing is more efficient. This also tells a cautionary tale that using artificial statistics of velocity, such as delta-correlated statistics, may be grossly misleading when dealing with real turbulence.

The arguments of the previous section can be applied even if the energy injection rate is not stationary. In Fig. 31, we presented simulations with intermittent driving checking relations $(75,76)$.

\subsection{Caveats in simulating astrophysical dynamo}

As we alluded above in Sect. 9.1, the timescales of initial growth of the magnetic field are extremely short compared to the dynamical timescale of the system-these two are separated by the factor of $\mathrm{Re}^{1 / 2}$, which is very large in astrophysical environments. Not so in numerical simulations, which are limited in terms of Re. This situation is further exacerbated by the small prefactor 0.0326 in the growth rate of $\gamma=0.0326 / \tau_{\eta}$. For example, simulation with $\mathrm{Re}=1600$ will result in characteristic growth time of $1 /\left(0.0326 \mathrm{Re}^{1 / 2}\right) \approx 0.77$ of the outer timescale of the system, while in astrophysical reality this is a negligible fraction of the outer timescale. ${ }^{1}$ The lesson from this is that if we take a very small initial magnetic field while simulating rather dynamically young object, e.g., collapsing cloud or a forming galaxy cluster, this may artificially delay the onset of the nonlinear dynamo and grossly underestimate the magnetic field at the end of the evolution (Beresnyak and Miniati 2016). This delay effect can also lead to the artificial dependence on the initial field value or direction, which should

\footnotetext{
1 See, e.g., how the growth in Fig. 30 starts with rather high values of $t$.
} 

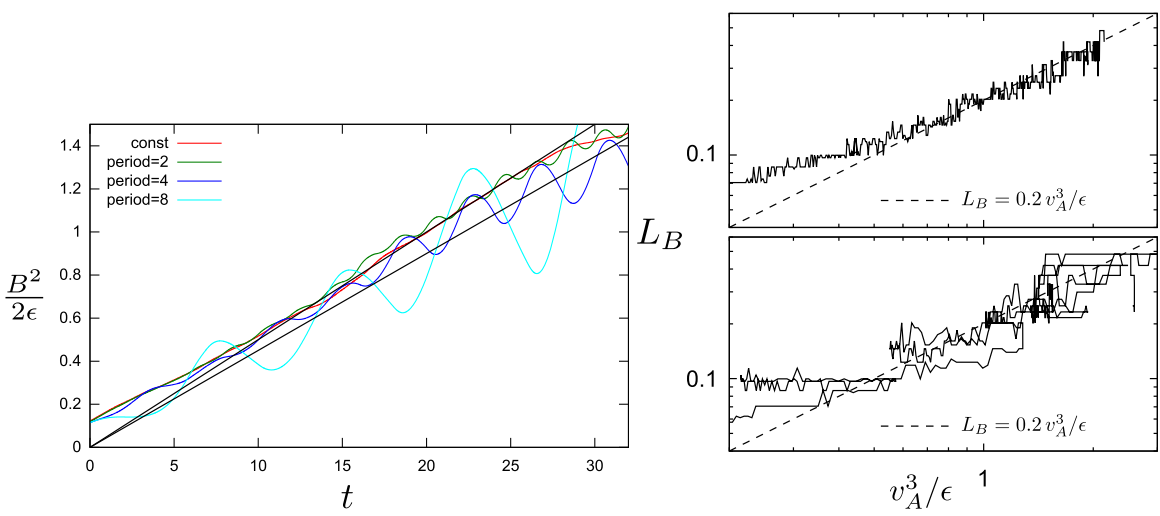

Fig. 31 Left: The magnetic energy in Alfvén units, divided by the energy driving rate, i.e., $B^{2} / 2 \varepsilon$ versus time (both in numerical time units, approximately correlation time $\tau_{c}$ ). Different curves correspond to intermittent driving with different periods. Right: The relation between magnetic energy and the outer scale. The upper plot corresponds to the case with constant driving (from Beresnyak 2012b), while the lower plot correspond to intermittent driving with the period of $8 \tau_{c}$. In this relation we used the dissipation rate $\varepsilon$ averaged over $2 \tau_{c}$. Outer scale was determined by the peak wavenumber of the spectrum. Best fit $c_{l} \approx 0.18$ in Eq. (76). Image reproduced with permission from Beresnyak and Miniati (2016); copyright by AAS

not normally appear in nonlinear dynamo, which, as any turbulence, erases any traces of the initial condition. The solution to this issue could be injecting initial field based on the amount of energy in the cascade, along the lines of Sect. 9.2.

Another issue is that popular ILES codes lack any knowledge or prescription of the microscales. While very often this is not an issue, because ILES code would simply absorb cascade energy into the thermal energy on the grid scales, but there is a qualitative difference between nature and an ILES simulation with zero initial field, which would produce zero magnetic field for all subsequent times. In nature, the small-scale non-ideal contributions to the induction equation, such as Biermann battery term, will always jump-start the dynamo and result in non-zero fields, also large $\mathrm{Re}$ in nature will ensure that average magnetization will be close to the estimate given by nonlinear dynamo in Sect. 9.2, i.e., the magnetic energy will be a certain fraction of the dissipated cascade energy. From MHD point of view, taking diffusivities to zero allows us to have two types of solutions - completely unmagnetized and fairly strongly magnetized, however, in nature only magnetized solution will be realized. ${ }^{2}$ We conclude that in dynamo situations ILES code should sometimes use subgrid dynamo prescription.

\subsection{Application to galaxy clusters}

Galaxy clusters constitute an interesting case of the small-scale dynamo. All properties of the cluster continue to evolve during the cosmic time, its mass also determining virial velocity which is always around the inflow velocity. Clusters are heated by the major

\footnotetext{
2 Assuming small-scale dynamo is indeed unstable, this requires certain minimal magnetic Reynolds number, but this is normally satisfied in most astrophysical environments.
} 
mergers, which is also the primary source of energy for the intracluster turbulence. On average, around 0.4 of heating comes from dissipation of solenoidal turbulence (Miniati and Beresnyak 2015). At the same time, as we saw in the previous section dynamo converts a fraction of 0.05 of this energy into magnetic energy. Thus we may conclude that magnetization $\beta$, the ratio of magnetic to thermal energy, should stay approximately constant through cosmic time, $\sim 40$ for the past $10 \mathrm{Gyr}$. Similarly, the outer magnetic scale will stay a constant fraction of the cluster virial radius, $\sim 1 / 200$.

\section{Compressible and supersonic turbulence}

The study of supersonic ISM turbulence is vital for understanding the structure of molecular clouds and subsequent star formation. In this respect, the studies of density scalings and thermal instability in DNS have become commonplace. One way to look at it is assuming that in addition to the Alfvén mode, additional compressible modes are excited as well. How this approach is valid in the regime where Alfvén waves couple strongly among themselves is still a matter of debate. Below we will show a mode decomposition techniques, as well as study density perturbations which are perturbed nonlinearly, by orders of magnitude in the supersonic regime.

Compressible turbulence is characterized by two dimensionless numbers, sonic Mach number $M_{s}=\delta v / c_{s}$ and Alfvénic Mach number $M_{A}=\delta v / v_{A}$. Plasma beta, the ratio of gas pressure to magnetic pressure can be expressed as $\beta=8 \pi P_{\text {gas }} / B^{2}=$ $2 \gamma M_{A}^{2} / M_{S}^{2}$. In a turbulent environment, these numbers tend to evolve towards "preferred" values, depending on the physics. For example in the absence of cooling, supersonic turbulence will heat itself to trans-sonic state $M_{s} \sim 1$ in one dynamical time. The persistent supersonic regime, therefore, is limited only to regions which have very efficient atomic or molecular cooling, such as molecular clouds, where $M_{s} \lesssim 10$.

Likewise, the Alfvénic Mach number is limited by the dynamo process that we described in Sect. 9. Turbulence with a very weak initial field tends to generate equipartition-strength fields, with $M_{A} \sim 1$, in around 20 dynamical times. We expect large (but not very large) $M_{A}$ only in dynamically young systems, such as in collapsing molecular clouds.

In galaxy clusters, which are continuously heated up by accretion and turbulence, its mass, and, therefore, virial velocity, also continue to increase. This leads to the situation when both $\delta v$ and $c_{s}$ are determined by virial velocity and $M_{s} \sim 1$. Due to the continuous growth of the cluster mass, it never approaches equipartition, so $M_{A} \sim 7$.

\subsection{Decomposition into modes}

Here we will use expressions for the phase wave speeds of the Alfvén and slow/fast modes $(37,38)$ from Sect. 5 and the same notation for $\cos \theta=(\hat{\mathbf{k}} \cdot \hat{\mathbf{B}})$. Our coordinate system will be defined by the unit vector of the magnetic field $\hat{\mathbf{B}}$, the unit vector of the Alfvén perturbation $\hat{\mathbf{e}}_{A}=\mathbf{k} \times \mathbf{B}_{0} /\left|\mathbf{k} \times \mathbf{B}_{0}\right|$, and the third unit vector perpendicular to the three, in the $(\mathbf{k}, \mathbf{B})$ plane: $\hat{\mathbf{e}}_{\perp}=\hat{\mathbf{e}}_{A} \times \hat{\mathbf{B}}_{0}$, see Fig. 32. 
Fig. 32 Coordinate system for vector decomposition into MHD modes. Note that all vectors except $\mathbf{e}_{A}$ are in the same plane

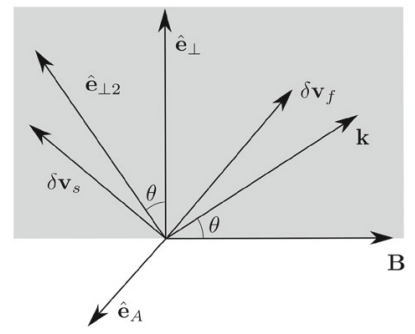

The perturbations of the velocity for the Alfvén mode are along $\hat{\mathbf{e}}_{A}$, also from induction equation $-\omega \delta \mathbf{B}=\mathbf{k} \times\left(\mathbf{v} \times \mathbf{B}_{\mathbf{0}}\right)$, however the sign of $\delta \mathbf{B}$ depend on the sign of $\omega$, i.e., whether the wave is propagating along the field direction, or in the opposite direction. The waves where $\delta \mathbf{B} \sim-\delta \mathbf{v}$ the waves propagate along the field. This also corresponds to $\delta w^{+}$propagating opposite to the field and $\delta w^{-}$along the field.

The perturbations of the velocity for the slow and fast mode are along mutually orthogonal vectors in the $(\mathbf{k}, \mathbf{B})$ plane:

$$
\delta \mathbf{v}_{f, s}=\hat{\mathbf{B}}\left(u_{f, s}^{2}-v_{A}^{2}\right) \cos \theta+\hat{\mathbf{e}}_{\perp} u_{f, s}^{2} \sin \theta .
$$

This two vectors and $\hat{\mathbf{e}}_{A}$ form an orthogonal coordinate system in wavenumber space. The perturbation $\delta \mathbf{B}$ for both modes is in the

$$
\delta \mathbf{B}_{f, s}=\hat{\mathbf{B}}_{0}-\hat{\mathbf{k}} \cos \theta
$$

direction, however again the sign of the contribution for each mode will depend on the direction of the wave propagation. Introducing $\Delta \mathbf{v}_{f, s}$ as perturbations where oppositely propagating wave contribute with different signs, the contribution to $\delta \mathbf{B}$ for each mode will be proportional to $\left|\Delta \mathbf{v}_{f, s} / u_{f, s}\right|$ (see, e.g., Cho and Lazarian 2003).

Alternatively, one can decompose in the coordinate system rotated around $\hat{\mathbf{e}}_{A}$ by $\theta$, which is made of $\hat{\mathbf{k}}, \hat{\mathbf{e}}_{A}$ and $\hat{\mathbf{e}}_{\perp 2}=\hat{\mathbf{e}}_{A} \times \hat{\mathbf{k}}$, in which case the velocity and magnetic field vectors along fast and slow modes can be expressed as

$$
\begin{aligned}
\delta \mathbf{v}_{f, s} & =\hat{\mathbf{k}}\left(u_{f, s}^{2} / v_{A}^{2}-\cos ^{2} \theta\right)+\hat{\mathbf{e}}_{\perp 2} \cos \theta \sin \theta, \\
\delta \mathbf{B}_{f, s} & =\hat{\mathbf{e}}_{\perp 2} .
\end{aligned}
$$

It was suggested in Cho and Lazarian $(2002,2003)$ that the Alfvén mode has an independent cascade (Sect. 7), slow mode is passive to Alfvén cascade and has the same spectra and anisotropy, and fast mode has an independent isotropic acoustic/wave turbulence cascade (Sect. 4.2):

$$
\begin{aligned}
& \text { Alfvén, Slow: } E^{A}(k) \propto k^{-5 / 3}, \quad k_{\|} \propto k_{\perp}^{2 / 3} . \\
& \text { Fast: } E^{f}(k) \propto k^{-3 / 2}, \text { isotropic spectrum. }
\end{aligned}
$$

This was broadly consistent with simulations in Cho and Lazarian (2002, 2003), however, steeper spectrum $\sim k^{-2}$ was reported in Kowal and Lazarian (2010) for fast 

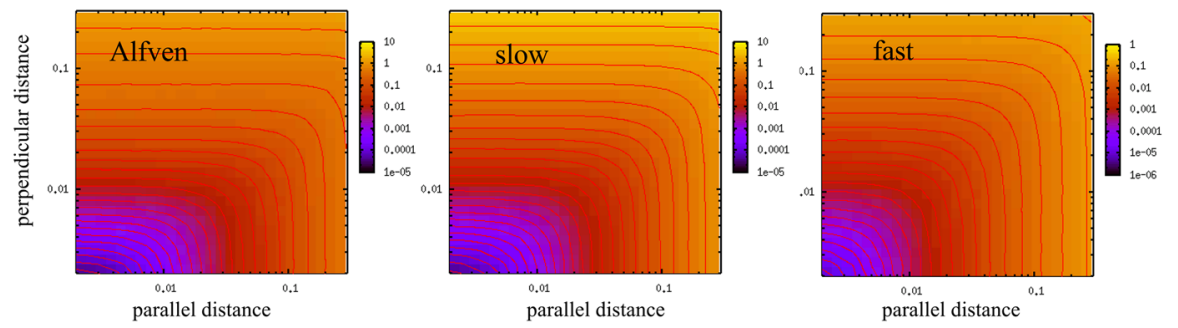

Fig. 33 Anisotropy of the Alfvén, slow and fast modes as evidenced by the contours of the second order structure function. Here we used the SF decomposition method. The Alfvén and slow mode exhibit scaledependent anisotropy, while the fast mode is almost isotropic. Image reproduced with permission from Beresnyak and Lazarian (2015); copyright by Springer

modes. Regarding the amplitude of each mode in realistic turbulence, it stronly depend on the way the turbulence is driven. For a particular incompressible driving of Cho and Lazarian $(2002,2003)$ they suggested a scaling relation

$$
\frac{\delta E_{\mathrm{fast}}}{\delta E_{\mathrm{Alf}}} \approx \frac{\delta V_{A} V_{A}}{V_{A}^{2}+c_{S}^{2}},
$$

where $\delta E_{\text {fast }}$ and $\delta E_{\text {Alf }}$ are energy of fast and Alfvén modes, respectively.

\subsection{Decomposition in real space}

Another way to decompose into modes is by using structure functions. In this method, the separation vector $\mathbf{l}$ of the structure function plays the role of the wavenumber, because of the correspondence between one-dimensional structure function along the particular line and the power spectrum along the same line (Sect. 3). Figure 33 shows the contours of the structure function corresponding to each mode obtained in datacubes from $M_{s}=10$ supersonic simulations used earlier in Beresnyak et al. (2005). The anisotropies of each mode show the same behavior as in the global decomposition method discussed above. The advantages in using the SF decomposition method is that it is a local measurement, so we can apply it even when the global average magnetic field is zero, e.g., it has been applied to the decomposition of MHD turbulence obtained in the high-resolution cosmological simulation of a galaxy cluster (Beresnyak et al. 2013).

\subsection{Density scalings}

The properties of density in supersonic turbulence is interesting to astronomers due to its relation to star formation. The density is primarily perturbed by the slow mode; however, in supersonic case, these perturbations are not small. Instead, density varies by several orders of magnitude within the box (Fig. 34). At the same time, the statistics of density is very different from the statistics of the slow mode velocity. It is worth noting that supersonic isothermal hydrodynamics predicts the log-normal distribution 
Fig. 34 Probability density function for a density in isothermal numerical simulations with $M_{A} \approx 1$ and various $M_{S}$. Image reproduced with permission from Beresnyak et al. (2005); copyright by AAS
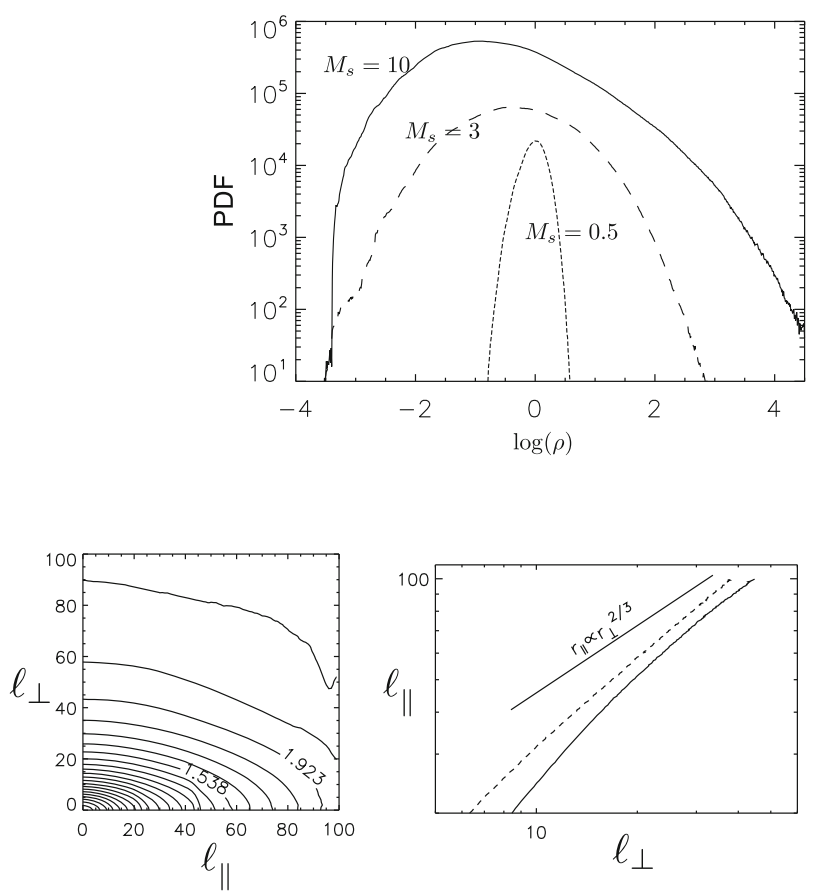

Fig. 35 Contours of the structure function of density (left), log-density (center) and the anisotropy of logdensity (right), solid line represents $M_{S} \sim 10$, dashed $M_{S} \sim 3$. Image reproduced with permission from Beresnyak et al. (2005); copyright by AAS

of density (Passot and Vázquez-Semadeni 1998) due to the gauge symmetry of logdensity in this case. This symmetry is broken in MHD. However, the PDF still resemble log-normal law (Fig. 35). Beresnyak et al. (2005) pointed out that in the low beta supersonic regime the perturbation of the slow mode velocity is almost along $\mathbf{B}_{0}$, so the dynamics of the slow mode is quasi-one-dimensional. This results in the generation of many slow shocks, which is indeed observed in simulations. The perturbations of density, e.g., its log-normal PDF, are created by these random slow shocks, similar to hydrodynamics. The Alfén mode only mixes these perturbations by shearing motions, without affecting PDF. On the other hand, the structure of density (SFs) is almost entirely determined by this shearing and is expected to have an anisotropic structure like velocity and magnetic field. In other words, two distinct physical processes act simultaneously and affect different statistical measures of the turbulent density field. The random multiplication of density induced by shocks, affect the PDFs, while the other, Alfvénic shearing, affects anisotropy and scaling of the structure function of the density. Revealing the structure created by shearing requires overcoming the effect of high-density clumps which will dominate $\operatorname{SF}^{2}(\rho)$. So, instead, we should use statistics of $\log (\rho)$, which have approximate Gaussian PDFs. This exercise is shown in Fig. 35. Indeed, the statistics of $\log (\rho)$ shows anisotropy characteristic of Alfvén shearing. 

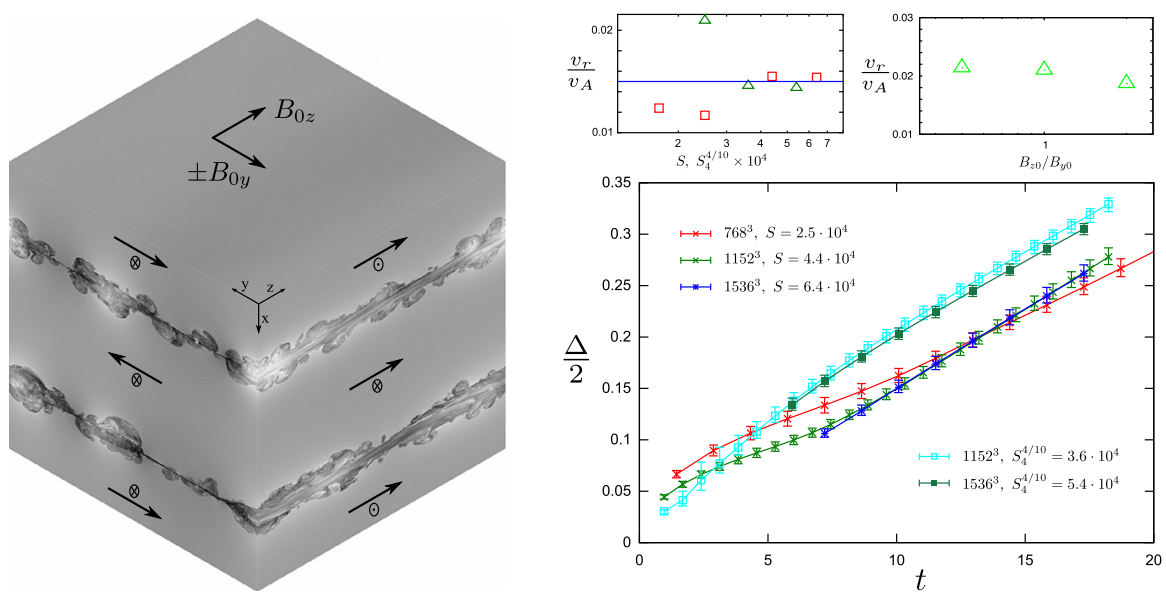

Fig. 36 Left: The setup of all-periodic reconnection with two current layers. The magnitude of the magnetic field is shown in grayscale. Right: the evolution of the layer width $\Delta$ (bottom) and the reconnection (mixing) rate as a function of the Lundquist number $S$ (top left) and the ratio of $B_{z 0} / B_{y 0}$ (top right). Image reproduced with permission from Beresnyak (2017); copyright by AAS

\section{Turbulence driven by magnetic field}

Turbulence in reconnection can appear as a result of instabilities, for example, resistive tearing (Biskamp 1986). Loureiro et al. (2007) demonstrated that the instability becomes faster and not slower with decreasing resistivity above a critical Lundquist number around $10^{4}$. Observing effects of the feedback of the release of magnetic energy in numerics is challenging because currently available 3D MHD numerics are limited by the Lundquist numbers of several of $10^{4}$.

Physically, periodic box simulations, like numerics in Beresnyak (2017) correspond to early times in the current sheet disruption when the outflow did not develop. Importantly enough, it did demonstrate fast (resistively-independent) reconnection rates, defined as mixing rates of the fluid. Simulations with open boundaries in Kowal et al. (2015) have been performed for a sufficiently long time to allow for the establishment of the stationary state. They correspond to later times when the stationary inflow/outflow appears.

One of the simplest setups to study the development of turbulence in the thin current layer is a periodic setup with the mean field $B_{z 0}$ threading the box, reconnecting field $\pm B_{y 0}$ changing the sign in the $x$ direction, see Fig. 36 . We consider the incompressible case, in which case the only dimensionless parameters of the problem are the Lundquist number $S$ and the ratio $B_{y 0} / B_{z 0}$. We use the planar sheet in an attempt to simulate a zoomed-in portion of a very large and unstable Sweet-Parker current layer. Lundquist number is defined with the box size, as $S=v_{A y} L / \eta$. We imagine that this box is a part of a bigger system with larger system size $L_{\text {global }}$. We aim to simulate early times, $t<L_{\text {global }} / V_{A}$, when the global outflow did yet develop. We also assume that the global Lundquist number, determined by the larger system, is asymptotically large so that we can ignore large-scale gradients. The simulation end is determined by the 

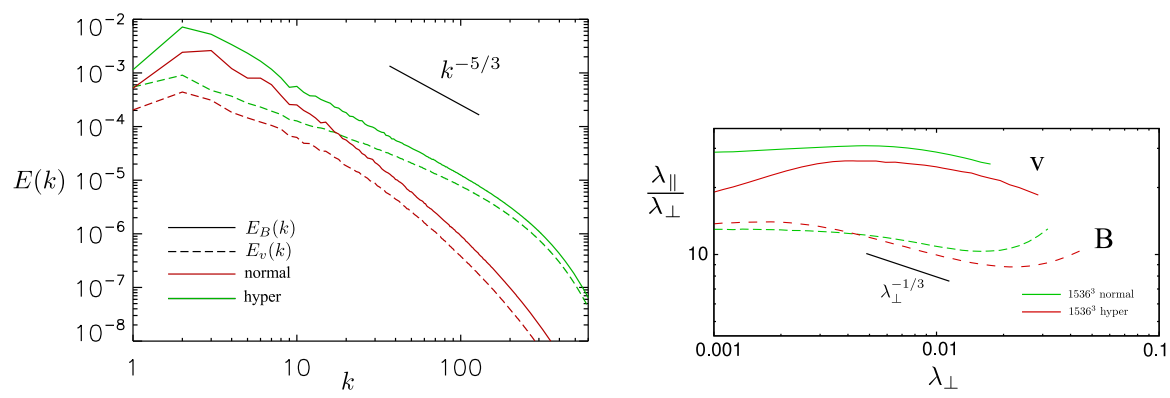

Fig. 37 Left: The y-z power spectra of velocity and magnetic perturbations of turbulence in the current layer. Right: Anisotropy from the ratio of parallel to perpendicular scales obtained from equating 2-order SFs (see Sect. 3.12). We used simulations with 2 nd as well as 4th order diffusivities (hyper diffusivities) to evaluate the effect of the dissipation on the statistics of turbulence in the layer. Images reproduced with permission from Beresnyak (2017); copright by AAS

development of structures with the size comparable to the box size, at which point our artificial periodic boundary starts influencing the result.

The free energy in the system is the energy density of the opposing fields $B_{y 0}^{2} / 8 \pi$, which is declining in the turbulent current layer due to dissipation. After $t \approx 0.3 \mathrm{~L} / v_{A z}$ the fraction of the dissipated energy $w_{d}$ becomes approximately constant, around $w_{d} \approx 0.4$. We calculate the reconnection rate as the speed of growth of the turbulent current layer width $\Delta$, i.e., we define $V_{r}=d \Delta / d t$. The evolution of $d$ and the inferred reconnection rate are shown in Fig. 36. $V_{r}$ was around $0.015 v_{A y}$ for high Lundquist numbers and is rather insensitive to the imposed mean field $B_{z 0}$ (Fig. 36). The dissipation rate per unit area of the current sheet can be calculated from $w_{d}$ and $v_{r}$ as

$$
\varepsilon_{S}=2 w_{d} v_{r}(1 / 2) \rho v_{A y}^{2} \approx 0.006 \rho v_{A y}^{3} .
$$

Note that we arrived at the expression not only for "fast reconnection" (independent on resistivity and viscosity) but also for "fast dissipation". This expression, modulo numerical coefficient, can be obtained by dimensional analysis using only $\rho$ and $v_{A y}$.

The field in the current layer can be analyzed statistically. We show the spectrum for one time slice in Fig. 37. The peak of the spectrum moves towards smaller wavenumbers, i.e., the outer scale of this turbulence is growing in time. This is unlike driven turbulence (Sect. 7) where this scale was determined by forcing and fixed. Another difference with driven turbulence is that magnetic spectrum is above kinetic on all scales, but closer to equipartition on smaller scales. This is similar to decaying MHD turbulence described, e.g., in Biskamp (2003). Qualitatively reconnection turbulence is very similar to decaying turbulence created by the initial random magnetic field.

Scale-locality is an important component of turbulent reconnection. Our spontaneous reconnection numerics corroborate scale-locality, because the spectral slope of perturbations is between -1 and -3 . In the real world, we expect the reconnection rate to be independent of system size as long as ion Larmor radius $r_{L}$ and ion skip depth $d_{i}$ are both much smaller than the layer width $\Delta$. On the right-hand side, Fig. 37 shows anisotropy expressed as a ratio of parallel to perpendicular scale $\lambda_{\|} / \lambda_{\perp}$, obtained by a method we explain in Sect. 7. We can also estimate the interaction strength param- 
eter $\xi=\delta v \lambda_{\|} / v_{A} \lambda_{\perp}$ and see that for this case it is around unity, i.e., we are dealing with critically balanced strong turbulence. Note that the anisotropy of our turbulence, being around $k_{\|} / k_{\perp} \sim 1 / 20$ is very different from the tangent of the fastest growing oblique tearing mode, $k_{\|} / k_{\perp}=B_{z} / B_{y}=1$. So turbulence forgets the properties of the oblique tearing that started it. From simulations with higher $B_{z}$ one also confirm Alfvén symmetry: increasing $B_{z}$ only increases parallel lengthscale, while keeping dynamics essentially unchanged (see Beresnyak 2017).

\section{Conclusion}

This review covers a set of topics that were chosen because they are either: (a) basic topics that are essential for the understanding of subsequent material or (b) have seen rapid progress recently, which is otherwise not covered in books or reviews. Due to this choice, many things, especially astrophysical and space applications of MHD turbulence, have been omitted or mentioned only in passing. This document, however, is a Living Review and will be evolving, below we overview several topics that we expect to add or expand. For the impatient, we mention older books and reviews that can be used to expand and deepen the reader's knowledge of the topic. Most of these have been already mentioned in the course of the review.

Mathematical tools to work with the statistical ensemble of turbulent realization can be found in the monograph by Monin and Yaglom (1975). Overviews of turbulence as a nonlinear dynamical process can be found in the books by Frisch (1995), Falkovich (2011), and Davidson (2015). A comprehensive, although older, book dedicated to MHD turbulence is Biskamp (2003), a few topics in MHD turbulence are also covered in Davidson (2013). An older book on mean-field dynamo theory is Krause and Raedler (1980), a more modern approach to the same topic, primarily for solar dynamo applications is the Living Review by Charbonneau (2010). A broader review on dynamo theory is Brandenburg and Subramanian (2005). In future editions, we plan to cover large-scale dynamo as well. For an in-depth review of solar wind turbulence, see the Living Review by Bruno and Carbone (2013). We plan to expand the section related to the solar wind and cover energy flux (MacBride et al. 2008) as well as magnetic helicity measurements (Brandenburg et al. 2011). An interesting case of energy cascade with applications to cosmological-scale magnetic fields and its dynamical evolution is a freely decaying homogeneous MHD turbulence, see, e.g. Brandenburg et al. (2015), which we also plan to cover in the future.

Several topics connecting astrophysical plasmas and MHD turbulence can be found in the book by Kulsrud (2005). In the ISM, MHD turbulence coexists with cosmic rays. Cosmic-ray interaction with MHD turbulence is a fairly large topic, for an introduction to cosmic rays as well as quasilinear scattering theory one can start with Schlickeiser (2002). One particularly important application of mutual interaction between cosmic rays and MHD turbulence, which will be covered in future editions of this review, is the acceleration of cosmic rays in supernova remnants. In front of strong shocks, MHD turbulence is self-generated by fast particles. This is supported by estimates of diffusion coefficient $D$ of cosmic rays in supernova remnants. $D$ in front of the shock is estimated to be many orders of magnitude smaller than $D$ in the ambient 
ISM, i.e., cosmic rays create their own MHD turbulence and dynamo and scatter themselves.

Supersonic turbulence with applications to ISM and star formations is covered in Mac Low and Klessen (2004) and McKee and Ostriker (2007). The physics of turbulent energy cascade in the supersonic case has been an open question for some time, but recently we saw progress in deriving exact analytic relations in supersonic case (Falkovich et al. 2010; Galtier and Banerjee 2011; Banerjee and Galtier 2013), as well as empirical findings and numerical verification (Wagner et al. 2012; Kritsuk et al. 2013). The earlier phenomenological approach of replacing statistics of velocity $\mathbf{u}$ with the statistics of $\rho^{1 / 3} \mathbf{u}$ in the compressible case (Fleck 1996; Kritsuk et al. 2007) have found a firmer foundation (Banerjee and Galtier 2014). This has also been used to explain observed statistical correlations, such as Larson's laws (Larson 1981) in star-forming clouds (Kritsuk et al. 2013).

Intermittency is the deviation from self-similarity of turbulence and is an important property that reminds us of the richness of the field of nonlinear fluid dynamics. While intermittency in hydrodynamics has been long studied as-is, the intermittency in different variables in MHD turbulence may give us something to think about its dynamical origin. On the other hand, extreme intermittency had been suggested to explain heating and molecular synthesis in the ISM. This will be covered in more detail in the future editions of this review. The numerical section will be expanded with mention of Lagrangian-Eulerian (moving mesh) codes and recent progress in this area. In future editions, we also will pay more attention to the connection between theory and observations. Big progress has been achieved in the area of cosmological structure formation by massive ab-initio simulations including $\Lambda \mathrm{CDM}$ initial conditions with $\Lambda$, dark matter and ordinary matter using grid refinement down to the scales of galaxies (e.g., the Illustris project). Some of these results are relevant to understand magnetization in filaments and, possibly, clusters and will be added later.

Open Access This article is distributed under the terms of the Creative Commons Attribution 4.0 International License (http://creativecommons.org/licenses/by/4.0/), which permits unrestricted use, distribution, and reproduction in any medium, provided you give appropriate credit to the original author(s) and the source, provide a link to the Creative Commons license, and indicate if changes were made.

\section{References}

Armstrong JW, Rickett BJ, Spangler SR (1995) Electron density power spectrum in the local interstellar medium. Astrophys J 443:209-221. https://doi.org/10.1086/175515

Banerjee S, Galtier S (2013) Exact relation with two-point correlation functions and phenomenological approach for compressible magnetohydrodynamic turbulence. Phys Rev E 87:013019. https://doi.org/ 10.1103/PhysRevE.87.013019. arXiv:1301.2470

Banerjee S, Galtier S (2014) A Kolmogorov-like exact relation for compressible polytropic turbulence. J Fluid Mech 742:230-242. https://doi.org/10.1017/jfm.2013.657

Beck R (2015) Magnetic fields in the nearby spiral galaxy IC 342: a multi-frequency radio polarization study. Astron Astrophys 578:A93. https://doi.org/10.1051/0004-6361/201425572. arXiv:1502.05439

Beresnyak A (2011) Spectral slope and Kolmogorov constant of MHD turbulence. Phys Rev Lett 106:075001. https://doi.org/10.1103/PhysRevLett.106.075001. arXiv:1011.2505 
Beresnyak A (2012a) Basic properties of magnetohydrodynamic turbulence in the inertial range. Mon Not R Astron Soc 422:3495-3502. https://doi.org/10.1111/j.1365-2966.2012.20859.x. arXiv:1111.5329

Beresnyak A (2012b) Universal nonlinear small-scale dynamo. Phys Rev Lett 108:035002. https://doi.org/ 10.1103/PhysRevLett.108.035002. arXiv:1109.4644

Beresnyak A (2014) Spectra of strong magnetohydrodynamic turbulence from high-resolution simulations. Astrophys J Lett 784:L20. https://doi.org/10.1088/2041-8205/784/2/L20

Beresnyak A (2015) On the parallel spectrum in magnetohydrodynamic turbulence. Astrophys J Lett 801:L9. https://doi.org/10.1088/2041-8205/801/1/L9. arXiv:1407.2613

Beresnyak A (2017) Three-dimensional spontaneous magnetic reconnection. Astrophys J 834:47. https:// doi.org/10.3847/1538-4357/834/1/47. arXiv:1301.7424

Beresnyak A, Lazarian A (2006) Polarization intermittency and its influence on MHD turbulence. Astrophys J Lett 640:L175-L178. https://doi.org/10.1086/503708. arXiv:astro-ph/0512315

Beresnyak A, Lazarian A (2008) Strong imbalanced turbulence. Astrophys J 682:1070-1075. https://doi. org/10.1086/589428. arXiv:0709.0554

Beresnyak A, Lazarian A (2009a) Comparison of spectral slopes of magnetohydrodynamic and hydrodynamic turbulence and measurements of alignment effects. Astrophys J 702:1190-1198. https://doi. org/10.1088/0004-637X/702/2/1190. arXiv:0812.0812

Beresnyak A, Lazarian A (2009b) Structure of stationary strong imbalanced turbulence. Astrophys J 702:460-471. https://doi.org/10.1088/0004-637X/702/1/460. arXiv:0904.2574

Beresnyak A, Lazarian A (2015) MHD turbulence, turbulent dynamo and applications. In: Lazarian A, de Gouveia Dal Pino E, Melioli C (eds) Magnetic fields in diffuse media, Astrophysics and Space Science Library, vol 407. Springer, Berlin, pp 163-226 arXiv:1406.1185

Beresnyak A, Lazarian A (2019) Turbulence in Magnetohydrodynamics, De Gruyter Studies in Mathematical Physics, vol 12. De Gruyter, Berlin, Boston

Beresnyak A, Miniati F (2016) Turbulent amplification and structure of the intracluster magnetic field. Astrophys J 817:127. https://doi.org/10.3847/0004-637X/817/2/127. arXiv:1507.00342

Beresnyak A, Lazarian A, Cho J (2005) Density scaling and anisotropy in supersonic magnetohydrodynamic turbulence. Astrophys J Lett 624:L93-L96. https://doi.org/10.1086/430702. arXiv:astro-ph/0502547

Beresnyak A, Jones TW, Lazarian A (2009) Turbulence-induced magnetic fields and structure of cosmic ray modified shocks. Astrophys J 707:1541-1549. https://doi.org/10.1088/0004-637X/707/2/1541. arXiv:0908.2806

Beresnyak A, Xu H, Li H, Schlickeiser R (2013) Magnetohydrodynamic turbulence and cosmic-ray reacceleration in galaxy clusters. Astrophys J 771:131. https://doi.org/10.1088/0004-637X/771/2/131. arXiv: 1301.7453

Biskamp D (1986) Magnetic reconnection via current sheets. Phys Fluids 29:1520-1531. https://doi.org/ $10.1063 / 1.865670$

Biskamp D (2003) Magnetohydrodynamic turbulence. Cambridge University Press, Cambridge

Boldyrev S (2005) On the spectrum of magnetohydrodynamic turbulence. Astrophys J Lett 626:L37-L40. https://doi.org/10.1086/431649. arXiv:astro-ph/0503053

Boldyrev S (2006) Spectrum of magnetohydrodynamic turbulence. Phys Rev Lett 96:115002. https://doi. org/10.1103/PhysRevLett.96.115002

Borovsky JE (2010) Contribution of strong discontinuities to the power spectrum of the solar wind. Phys Rev Lett 105:111102. https://doi.org/10.1103/PhysRevLett.105.111102

Brandenburg A, Subramanian K (2005) Astrophysical magnetic fields and nonlinear dynamo theory. Phys Rep 417:1-209. https://doi.org/10.1016/j.physrep.2005.06.005. arXiv:astro-ph/0405052

Brandenburg A, Subramanian K, Balogh A, Goldstein ML (2011) Scale dependence of magnetic helicity in the solar wind. Astrophys J 734:9. https://doi.org/10.1088/0004-637X/734/1/9

Brandenburg A, Kahniashvili T, Tevzadze AG (2015) Nonhelical inverse transfer of a decaying turbulent magnetic field. Phys Rev Lett 114:075001. https://doi.org/10.1103/PhysRevLett.114.075001. arXiv: 1404.2238

Bruno R, Carbone V (2013) The solar wind as a turbulence laboratory. Living Rev Sol Phys 2:4. https:// doi.org/10.12942/lrsp-2005-4

Charbonneau P (2010) Dynamo models of the solar cycle. Living Rev Sol Phys 7:3. https://doi.org/10. 12942/1rsp-2010-3

Chen CHK, Bale SD, Salem CS, Maruca BA (2013) Residual energy spectrum of solar wind turbulence. Astrophys J 770:125. https://doi.org/10.1088/0004-637X/770/2/125 
Chepurnov A, Lazarian A (2010) Extending the big power law in the sky with turbulence spectra from Wisconsin H $\alpha$ Mapper Data. Astrophys J 710:853-858. https://doi.org/10.1088/0004-637X/710/1/ 853. arXiv:0905.4413

Cho J, Lazarian A (2002) Compressible sub-Alfvénic MHD turbulence in low- $\beta$ plasmas. Phys Rev Lett 88:245001. https://doi.org/10.1103/PhysRevLett.88.245001. arXiv:astro-ph/0205282

Cho J, Lazarian A (2003) Compressible magnetohydrodynamic turbulence: mode coupling, scaling relations, anisotropy, viscosity-damped regime and astrophysical implications. Mon Not R Astron Soc 345:325-339. https://doi.org/10.1046/j.1365-8711.2003.06941.x. arXiv:astro-ph/0301062

Cho J, Vishniac ET (2000) The anisotropy of magnetohydrodynamic Alfvénic turbulence. Astrophys J 539:273-282. https://doi.org/10.1086/309213

Cho J, Vishniac ET, Beresnyak A, Lazarian A, Ryu D (2009) Growth of magnetic fields induced by turbulent motions. Astrophys J 693:1449-1461. https://doi.org/10.1088/0004-637X/693/2/1449. arXiv:0812.0817

Corrsin S (1963) Estimates of the relations between Eulerian and Lagrangian scales in large Reynolds number turbulence. J Atmosph Sci 20:115-119. https://doi.org/10.1175/1520-0469(1963)020<0115: EOTRBE $>2.0 . \mathrm{CO} ; 2$

Cowling TG (1933) The magnetic field of sunspots. Mon Not R Astron Soc 94:39-48. https://doi.org/10. 1093/mnras/94.1.39

Davidson PA (2013) Turbulence in rotating, stratified and electrically conducting fluids. Cambridge University Press, Cambridge

Davidson PA (2015) Turbulence: an introduction for scientists and engineers. Oxford University Press, Oxford. https://doi.org/10.1093/acprof:oso/9780198722588.001.0001

Dolag K, Stasyszyn F, Donnert J, Pakmor R (2009) Magnetic fields and cosmic rays in galaxy clusters and large scale structures. In: Strassmeier KG, Kosovichev AG, Beckman JE (eds) Cosmic magnetic fields: from planets, to stars and galaxies. IAU symposium, vol 259, Cambridge University Press, Cambridge, pp 519-528. https://doi.org/10.1017/S1743921309031202

Falkovich G (2011) Fluid mechanics: a short course for physicists. Cambridge University Press, Cambridge. https://doi.org/10.1017/CBO9780511794353

Falkovich G, Fouxon I, Oz Y (2010) New relations for correlation functions in Navier-Stokes turbulence. J Fluid Mech 644:465. https://doi.org/10.1017/S0022112009993429. arXiv:0909.3404

Feretti L, Dallacasa D, Govoni F, Giovannini G, Taylor GB, Klein U (1999) The radio galaxies and the magnetic field in Abell 119. Astron Astrophys 344:472-482 arXiv:astro-ph/9902019

Fleck RC Jr (1996) Scaling relations for the turbulent, non-self-gravitating, neutral component of the interstellar medium. Astrophys J 458:739. https://doi.org/10.1086/176853

Frisch U (1995) Turbulence. The legacy of A. N. Kolmogorov. Cambridge University Press, Cambridge

Galtier S, Banerjee S (2011) Exact relation for correlation functions in compressible isothermal turbulence. Phys Rev Lett 107:134501. https://doi.org/10.1103/PhysRevLett.107.134501. arXiv:1108.4529

Galtier S, Nazarenko SV, Newell AC, Pouquet A (2000) A weak turbulence theory for incompressible magnetohydrodynamics. J Plasma Phys 63:447-488. https://doi.org/10.1017/S0022377899008284. arXiv:astro-ph/0008148

Galtier S, Nazarenko SV, Newell AC, Pouquet A (2002) Anisotropic turbulence of Shear-Alfvén waves. Astrophys J Lett. 564:L49-L52. https://doi.org/10.1086/338791

Gogoberidze G (2007) On the nature of incompressible magnetohydrodynamic turbulence. Phys Plasmas 14:022304. https://doi.org/10.1063/1.2437753. arXiv:astro-ph/0611894

Goldreich P, Sridhar S (1995) Toward a theory of interstellar turbulence. 2: Strong Alfvénic turbulence. Astrophys J 438:763-775. https://doi.org/10.1086/175121

Gotoh T, Fukayama D, Nakano T (2002) Velocity field statistics in homogeneous steady turbulence obtained using a high-resolution direct numerical simulation. Phys Fluids 14:1065. https://doi.org/10.1063/1. 1448296

Grant HL, Stewart RW, Moilliet A (1962) Turbulence spectra from a tidal channel. J Fluid Mech 12:241-268. https://doi.org/10.1017/S002211206200018X

Grappin R, Müller WC (2010) Scaling and anisotropy in magnetohydrodynamic turbulence in a strong mean magnetic field. Phys Rev E 82:026406. https://doi.org/10.1103/PhysRevE.82.026406. arXiv:1008.0727

Grappin R, Velli M (1996) Waves and streams in the expanding solar wind. J Geophys Res 101:425-444. https://doi.org/10.1029/95JA02147 
Haugen NE, Brandenburg A, Dobler W (2004) Simulations of nonhelical hydromagnetic turbulence. Phys Rev E 70:016308. https://doi.org/10.1103/PhysRevE.70.016308. arXiv:astro-ph/0307059

Horbury TS, Forman M, Oughton S (2008) Anisotropic scaling of magnetohydrodynamic turbulence. Phys Rev Lett 101:175005. https://doi.org/10.1103/PhysRevLett.101.175005. arXiv:0807.3713

Iroshnikov PS (1964) Turbulence of a conducting fluid in a strong magnetic field. Sov Astron 7:566

Jiang YF, Stone JM, Davis SW (2019) Super-Eddington accretion disks around supermassive black holes. Astrophys J 880:67. https://doi.org/10.3847/1538-4357/ab29ff. arXiv:1709.02845

Kadomtsev BB, Pogutse OP (1974) Nonlinear helical perturbations of a plasma in the tokamak. Sov Phys JETP 38:283

Kaneda Y, Ishihara T, Yokokawa M, Itakura K, Uno A (2003) Energy dissipation rate and energy spectrum in high resolution direct numerical simulations of turbulence in a periodic box. Phys Fluids 15:L21-L24. https://doi.org/10.1063/1.1539855

Käpylä PJ, Korpi MJ, Brandenburg A (2009) Alpha effect and turbulent diffusion from convection. Astron Astrophys 500:633-646. https://doi.org/10.1051/0004-6361/200811498. arXiv:0812.1792

Kazantsev AP (1968) Enhancement of a Magnetic Field by a Conducting Fluid. Sov Phys JETP 26:1031

Kolmogorov A (1941) The local structure of turbulence in incompressible viscous fluid for very large Reynolds' Numbers. Dokl Akad Nauk SSSR 30:301-305

Kowal G, Lazarian A (2010) Velocity field of compressible magnetohydrodynamic turbulence: wavelet decomposition and mode scalings. Astrophys J 720:742-756. https://doi.org/10.1088/0004-637X/ 720/1/742

Kowal G, Falceta-Gonçalves DA, Lazarian A, Vishniac ET (2015) Turbulence generated by reconnection. preprint ask author if this is Kowal et al 2017 ApJ 83891

Kraichnan R (1965) Inertial-range spectrum of hydromagnetic turbulence. Phys Fluids 8:1385. https://doi. org/10.1063/1.1761412

Kraichnan RH, Nagarajan S (1967) Growth of turbulent magnetic fields. Phys Fluids 10:859-870. https:// doi.org/10.1063/1.1762201

Krause F, Raedler KH (1980) Mean-field magnetohydrodynamics and dynamo theory. Pergamon Press, Oxford

Kritsuk AG, Norman ML, Padoan P, Wagner R (2007) The statistics of supersonic isothermal turbulence. Astrophys J 665:416-431. https://doi.org/10.1086/519443. arXiv:0704.3851

Kritsuk AG, Lee CT, Norman ML (2013) A supersonic turbulence origin of Larson's laws. Mon Not R Astron Soc 436:3247-3261. https://doi.org/10.1093/mnras/stt1805. arXiv:1309.5926

Kulsrud RM (2005) Plasma physics for astrophysics. Princeton Series in Astrophysics, Princeton University Press, Princeton

Kulsrud RM, Anderson SW (1992) The spectrum of random magnetic fields in the mean field dynamo theory of the Galactic magnetic field. Astrophys J 396:606-630. https://doi.org/10.1086/171743

Laing RA, Bridle AH, Parma P, Murgia M (2008) Structures of the magnetoionic media around the FanaroffRiley Class I radio galaxies 3C31 and Hydra A. Mon Not R Astron Soc 391:521-549. https://doi.org/ 10.1111/j.1365-2966.2008.13895.x. arXiv:0809.2411

Landau LD, Lifshitz EM (1959) Fluid mechanics. Pergamon Press, Oxford

Landau LD, Lifshitz EM (1960) Electrodynamics of continuous media. Pergamon Press, Oxford

Larson RB (1981) Turbulence and star formation in molecular clouds. Mon Not R Astron Soc 194:809-826. https://doi.org/10.1093/mnras/194.4.809

Lithwick Y, Goldreich P, Sridhar S (2007) Imbalanced strong MHD turbulence. Astrophys J 655:269-274. https://doi.org/10.1086/509884. arXiv:astro-ph/0607243

Loureiro NF, Schekochihin AA, Cowley SC (2007) Instability of current sheets and formation of plasmoid chains. Phys Plasmas 14:100703. https://doi.org/10.1063/1.2783986

Mac Low MM, Klessen RS (2004) Control of star formation by supersonic turbulence. Rev Mod Phys 76:125-194. https://doi.org/10.1103/RevModPhys.76.125. arXiv:astro-ph/0301093

MacBride BT, Smith CW, Forman MA (2008) The turbulent cascade at 1 au: energy transfer and the third-order scaling for MHD. Astrophys J 679:1644. https://doi.org/10.1086/529575

Maron J, Goldreich P (2001) Simulations of incompressible magnetohydrodynamic turbulence. Astrophys J 554:1175-1196. https://doi.org/10.1086/321413. arXiv:astro-ph/0012491

Mason J, Cattaneo F, Boldyrev S (2006) Dynamic alignment in driven magnetohydrodynamic turbulence. Phys Rev Lett 97:255002. https://doi.org/10.1103/PhysRevLett.97.255002. arXiv:astro-ph/0602382 
McComas DJ, Barraclough BL, Funsten HO, Gosling JT, Santiago-Muñoz E, Skoug RM, Goldstein BE, Neugebauer M, Riley P, Balogh A (2000) Solar wind observations over Ulysses' first full polar orbit. J Geophys Res 105:10419-10433. https://doi.org/10.1029/1999JA000383

McKee CF, Ostriker EC (2007) Theory of star formation. Annu Rev Astron Astrophys 45:565-687. https:// doi.org/10.1146/annurev.astro.45.051806.110602. arXiv:0707.3514

Miniati F, Beresnyak A (2015) Self-similar energetics in large clusters of galaxies. Nature 523:59-62. https://doi.org/10.1038/nature14552. arXiv:1507.01940

Monin AS, Yaglom AM (1975) Statistical fluid mechanics: mechanics of turbulence, vol 2, rev and enl edn. MIT Press, Cambridge

Müller WC, Grappin R (2005) Spectral energy dynamics in magnetohydrodynamic turbulence. Phys Rev Lett 95:114502. https://doi.org/10.1103/PhysRevLett.95.114502. arXiv:physics/0509019

Passot T, Vázquez-Semadeni E (1998) Density probability distribution in one-dimensional polytropic gas dynamics. Phys Rev E 58:4501-4510. https://doi.org/10.1103/PhysRevE.58.4501. arXiv:physics/9802019

Perez JC, Boldyrev S (2009) Role of cross-helicity in magnetohydrodynamic turbulence. Phys Rev Lett 102:025003. https://doi.org/10.1103/PhysRevLett.102.025003. arXiv:0807.2635

Politano H, Pouquet A (1998) von Kármán-Howarth equation for magnetohydrodynamics and its consequences on third-order longitudinal structure and correlation functions. Phys Rev E 57:R21-R24. https://doi.org/10.1103/PhysRevE.57.R21

Ryu D, Kang H, Cho J, Das S (2008) Turbulence and magnetic fields in the large-scale structure of the universe. Science 320:909. https://doi.org/10.1126/science.1154923. arXiv:0805.2466

Schekochihin AA, Cowley SC (2007) Turbulence and magnetic fields in astrophysical plasmas. In: Molokov S, Moreau R, Moffatt K (eds) Magnetohydrodynamics: fluid mechanics and its applications. Springer, Dordrecht, pp 85-115. https://doi.org/10.1007/978-1-4020-4833-3_6

Schekochihin AA, Cowley SC, Taylor SF, Maron JL, McWilliams JC (2004) Simulations of the Small-Scale Turbulent Dynamo. Astrophys J 612:276-307. https://doi.org/10.1086/422547. arXiv:astro-ph/0312046

Schekochihin AA, Cowley SC, Dorland W, Hammett GW, Howes GG, Quataert E, Tatsuno T (2009) Astrophysical gyrokinetics: kinetic and fluid turbulent cascades in magnetized weakly collisional plasmas. Astrophys J Suppl 182:310-377. https://doi.org/10.1088/0067-0049/182/1/310. arXiv:0704.0044

Schlickeiser R (2002) Cosmic ray astrophysics. Springer, Berlin. https://doi.org/10.1007/978-3-66204814-6

Schlüter A, Biermann I (1950) Interstellare Magnetfelder. Z Naturforsch 5:237

Sreenivasan K (1995) On the universality of the Kolmogorov constant. Phys Fluids 7:2778. https://doi.org/ $10.1063 / 1.868656$

Strauss HR (1976) Nonlinear, three-dimensional magnetohydrodynamics of noncircular tokamaks. Phys Fluids 19:134-140. https://doi.org/10.1063/1.861310

Tenerani A, Velli M (2017) Evolving waves and turbulence in the outer corona and inner heliosphere: the accelerating expanding box. Astrophys J 843:26. https://doi.org/10.3847/1538-4357/aa71b9. arXiv: 1702.03014

Tennekes H, Lumley JL (1972) First course in turbulence. MIT Press, Cambridge

Vainshtein SI, Zeldovich YB (1972) Origin of magnetic fields in astrophysics (turbulent "dynamo" mechanisms). Phys Usp 15:159-172

Vincenzi D (2002) The Kraichnan-Kazantsev dynamo. J Stat Phys 106:1073-1091. https://doi.org/10.1023/ A:1014089820881

Vishniac ET, Cho J (2001) Magnetic helicity conservation and astrophysical dynamos. Astrophys J 550:752760. https://doi.org/10.1086/319817. arXiv:astro-ph/0010373

Vishniac ET, Lazarian A, Cho J (2003) Problems and Progress in Astrophysical Dynamos. In: Falgarone E, Passot T (eds) Turbulence and Magnetic Fields in Astrophysics, Lecture Notes in Physics, vol 614. Springer, Berlin, pp 376-401 arXiv:astro-ph/0205557

Wagner R, Falkovich G, Kritsuk AG, Norman ML (2012) Flux correlations in supersonic isothermal turbulence. J Fluid Mech 713:482-490. https://doi.org/10.1017/jfm.2012.470. arXiv:1209.2164

Wicks RT, Horbury TS, Chen CHK, Schekochihin AA (2011) Anisotropy of imbalanced Alfvénic turbulence in fast solar wind. Phys Rev Lett 106:045001. https://doi.org/10.1103/PhysRevLett.106.045001

Wicks RT, Mallet A, Horbury TS, Chen CHK, Schekochihin AA, Mitchell JJ (2013) Alignment and scaling of large-scale fluctuations in the solar wind. Phys Rev Lett 110:025003. https://doi.org/10.1103/ PhysRevLett.110.025003. arXiv:1209.5362 
Yeung PK, Zhou Y (1997) Universality of the Kolmogorov constant in numerical simulations of turbulence. Phys Rev E 56:1746-1752. https://doi.org/10.1103/PhysRevE.56.1746

Zakharov VE, Lvov VS, Falkovich G (1992) Kolmogorov spectra of turbulence I. Wave turbulence, Springer Series in Nonlinear Dynamics, vol 10. Springer, Berlin. https://doi.org/10.1007/978-3-642-50052-7

Publisher's Note Springer Nature remains neutral with regard to jurisdictional claims in published maps and institutional affiliations. 